\title{
APLICACIÓN DE LAS MÁQUINAS DE SOPORTE VECTORIAL AL RECONOCIMIENTO DE HABLANTES
}

\author{
UNIVERSIDAD AUTÓNOMA METROPOLITANA \\ MAESTRÍA EN CIENCIAS Y TECNOLOGÍAS DE LA INFORMACIÓN \\ Juan Gabriel Pedroza Bernal \\ pedrozafm@yahoo.com.mx \\ Asesores de Investigación: \\ Dr. Alfonso Prieto Guerrero \\ Dr. John Henry Goddard Close
}

22 de Junio 2007 


\section{Índice general}

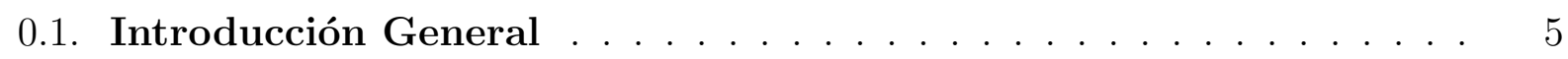

1. Verificación de Hablantes 9

1.1. Introducción . . . . . . . . . . . . . . . . . . . . . 9 9

1.2. Esquema General de Verificación de Hablantes . . . . . . . . . . . . . . 10

1.3. Estado del Arte . . . . . . . . . . . . . . . . . . . . . . . 11

2. Sistema Automático de Verificación $\quad 19$

2.1. Metodología . . . . . . . . . . . . . . . . . . . . . . . . . . . . . . . . 19

2.2. Base de Registros de Voz . . . . . . . . . . . . . . . . . . . . 20

2.2.1. Protocolo de Grabación . . . . . . . . . . . . . . . . . . . 23

2.2.2. Análisis Fonológico y Silábico . . . . . . . . . . . . . . . . 28

2.3. Procesamiento de la Voz . . . . . . . . . . . . . . . . . . . . . . . 30

2.3.1. Supresión de Silencios . . . . . . . . . . . . . . . . . . . . 32

2.3.2. Banco de Filtros Mel . . . . . . . . . . . . . . . . . . . . . 37

2.4. Máquinas de Soporte Vectorial . . . . . . . . . . . . . . . . . . . . . 38

2.4.1. Conjuntos Separables Linealmente . . . . . . . . . . . . . . . . 39

2.4.2. Conjuntos No Separables Linealmente . . . . . . . . . . . . . . . . 42

3. Desempeño del Sistema 47

3.1. Entrenamiento y Pruebas . . . . . . . . . . . . . . . . . . . . . . 48

3.1.1. Entrenamiento del Sistema . . . . . . . . . . . . . . . . 50

3.1.2. Pruebas y Resultados . . . . . . . . . . . . . . . . . . . . 51

3.2. Curvas DET . . . . . . . . . . . . . . . . . . . . 55

3.3. Comprobación con Voz en Tiempo Real . . . . . . . . . . . . . . . . . 59

3.3.1. Pruebas y Resultados . . . . . . . . . . . . . . . . . . . . . . 59

3.3.2. Curvas DET con Voz en Tiempo Real . . . . . . . . . . . . . . 65

3.4. Resultados Recientes con SVM . . . . . . . . . . . . . . . . 67

3.5. Validación del Supresor de Silencios . . . . . . . . . . . . . . . . . 70 
4. Otros Sistemas de Clasificación $\quad 75$

4.1. Modelos de Mezclas Gaussianas . . . . . . . . . . . . . . . . . . . . 75

4.1.1. Pruebas y Resultados . . . . . . . . . . . . . . . . . . . . . . . . . . . . . . . . . . . .

4.1.2. Curvas DET del Sistema GMM . . . . . . . . . . . . . . . . . 82

4.2. Redes Neuronales Artificiales . . . . . . . . . . . . . . . . . . 85

4.2.1. Entrenamiento, Pruebas y Resultados . . . . . . . . . . . . . . 88

4.2.2. Curvas DET del sistema ANN . . . . . . . . . . . . . . . . . . . 93

5. Conclusiones y Trabajo Futuro $\quad 97$

5.1. Conclusiones . . . . . . . . . . . . . . . . . . . . . 97

5.2. Trabajo Futuro . . . . . . . . . . . . . . . . 100

$\begin{array}{ll}\text { A. Anexos } & 101\end{array}$

A.1. Notación SAMPA del Español . . . . . . . . . . . . . . . . . . . . . 101

A.2. Algoritmo de Cálculo de Curvas DET . . . . . . . . . . . . . . . . . . . 102

$\begin{array}{ll}\text { Referencias } & 105\end{array}$ 


\subsection{Introducción General}

Actualmente es difícil concebir un sistema de cualquier índole, sin considerar aspectos relacionados con la seguridad del mismo. El término de seguridad, a pesar de tener diferentes acepciones, guarda la idea de preservar las condiciones de un estado considerado normal o aceptable. Así, la relación entre el reconocimiento de personas y la seguridad tiene un margen amplio, pues puede encontrarse tanto en aplicaciones forenses o legales como en controles de acceso a algún sistema; por ejemplo para determinar la identidad de una persona, su participación en un hecho, la validez de una petición de acceso, etc., en cualquier caso resulta evidente la necesidad de determinar la certeza o falsedad en la identidad de una persona.

El reconocimiento requiere de una base de registros conformada por algún patrón de referencia, que sea único para cada persona. Con esta base de registros se puede, conjuntamente con algún sistema automático y/o mediante alguna metodología, determinar si dada una persona cualquiera, ésta cuenta con registro o no y en su caso exhibir su identidad. Dos problemas fundamentales que enfrenta la realización de un sistema de reconocimiento son: establecer y definir la(s) característica(s) que serán consideradas como únicas para cada persona y determinar el(los) procedimiento(s) o metodología(s) mediante los cuales se realizará el reconocimiento. Al respecto, la biometría ha proporcionado el sustento científico para realizar sistemas de reconocimiento automático, basándose en la medición de propiedades fisiológicas del cuerpo humano como son las huellas digitales, el iris del ojo y también la voz.

En particular el reconocimiento mediante voz, se diferencia de los anteriores en que la voz no tiene, a priori, alguna característica que pueda ser considerada como única y en que la representación matemática de señales de voz es considerada como un proceso aleatorio, lo cual modifica drásticamente la naturaleza del problema de reconocimiento, pues las huellas digitales o el iris del ojo, son considerados en general como patrones gráficos fijos, al igual que otros como la forma de la cara o la de las manos. La necesidad de considerar un tratamiento de la señal de voz como un proceso aleatorio, estriba principalmente en los siguientes hechos:

- Físicamente, la voz es una onda mecánica de sonido que se propaga en el medio y es por ello continua, sin embargo su representación a través de cualquier sistema computacional es en forma discreta. La técnica que permite esta transformación es llamada muestreo y los datos obtenidos del mismo, dada una señal, no son predecibles a priori.

- La señal de voz guarda un alto grado de variabilidad en sus características para una misma persona, pues ésta es influenciada por factores ambientales y emocionales. Adicionalmente, los sistemas de grabado de voz provocan, de forma intrínseca, una distorsión en la señal recibida. 
Debido a la trascendencia de este tipo de sistemas de reconocimiento y al interés científico que generan, en diferentes países existen esfuerzos de investigación donde se aplican técnicas y metodologías para proporcionar sistemas cada vez más seguros y confiables y con mejores parámetros de desempeño. Sin embargo la mayoría de estos trabajos han sido desarrollados en idiomas diferentes al español de México, por lo que este trabajo presenta aspectos específicos del desarrollo de sistemas de reconocimiento en nuestro idioma. Adicionalmente se plantea la aplicación de las Máquinas de Soporte Vectorial, como una técnica de clasificación recientemente utilizada y de la cual se tienen expectativas favorables. También se describe la conformación de una base de registros de voz en español bajo la cual se realiza la implementación de un sistema de verificación. Esta base de registros de voz sienta un precedente que puede ser aprovechado en futuras investigaciones en esta materia. Parte esencial del trabajo es la descripción del procesamiento al que fue sometida la voz, hasta obtener información representativa de la misma.

De esta forma el objetivo general de la aplicación que se presenta, consiste en la implementación y evaluación del desempeño de un sistema de verificación de hablantes, basado en Máquinas de Soporte Vectorial como sistema de clasificación. Esta implementación debe tratar, principalmente, con la variabilidad de la voz, con la influencia de factores intrínsecos (como la del módulo de grabación) y con el ruido presente en el ambiente. De igual forma debe considerar aspectos relacionados con la representación y representatividad de la voz, así como su procesamiento hasta la conformación de un modelo. Un objetivo adicional es la evaluación del sistema, para ello se requiere comparar su desempeño con el obtenido mediante otro sistema de clasificación. La comparación del desempeño requiere analizar el error obtenido en los sistemas, lo cual permite concluir sobre su comportamiento.

Con base en los objetivos planteados, este documento se estructura de la siguiente forma:

En el primer capítulo se presenta un esquema general sobre los sistemas de verificación por voz. También un estado del arte sobre los sistemas de reconocimiento por voz, el cual hace énfasis en diferentes desarrollos científicos y comerciales en esta área, así como sus características principales y destaca sus parámetros de desempeño. En el capítulo 2 se describe la conformación de una base de registros de voz que considera diferentes aspectos de la variabilidad de la voz y permite la operación de un sistema de reconocimiento así como la valoración del desempeño del mismo. Posteriormente se expone el procesamiento al que son sometidos los registros de voz, hasta la obtención de los llamados vectores de características, los cuales sintetizan el contenido frecuencial de la voz para cada hablante. Se finaliza este capítulo con una amplia descripción de la teoría en la que se basan las Máquinas de Soporte Vectorial como una técnica que permite la clasificación o separación de conjuntos de vectores. En el capítulo 3 se describe el procedimiento seguido para realizar el entrenamiento del sistema de clasificación así como las pruebas que lo evalúan. 
Adicionalmente se presenta el comportamiento del sistema con voz en tiempo real. Con el objetivo de comparar los resultados obtenidos mediante el uso de Máquinas de Soporte Vectorial como sistema de clasificación, en el capítulo 4 se presentan dos metodologías de clasificación adicionales; Modelos de Mezclas Gaussianas y Redes Neuronales. Ambas son implementadas sobre los datos de los registros procesados. Por último, en el capítulo 5, se dan las conclusiones pertinentes y las actividades que pueden derivar de forma inmediata del presente trabajo. 


\section{Capítulo 1}

\section{Verificación de Hablantes}

\subsection{Introducción}

El reconocimiento de hablantes es un área que depende en gran parte del procesamiento de señales y que se ocupa de la detección, identificación y verificación automática de hablantes, dichos conceptos son descritos a continuación:

Detección: Consiste en determinar a partir de la voz de un individuo determinado, si éste participó o no en una secuencia o trama de voz grabada. Un sistema de detección establece la participación de una persona en una grabación dada.

Identificación: Consiste en determinar la identidad de un individuo a partir de su voz; el individuo proporciona su voz al sistema el cual determina el nombre del hablante o si éste es desconocido.Un sistema de identificación determina la identidad de una persona a partir de su voz o en su caso indica que el hablante es desconocido.

Verificación: Consiste en determinar si un individuo tiene permiso de acceso o no a partir de su voz, de acuerdo a una base de patrones de voz establecida previamente. Un sistema de verificación determina si la voz proporcionada es de quien dice ser.

Existe trabajo considerable realizado en diferentes organizaciones e instituciones en reconocimiento de hablantes; universidades, industria y laboratorios de diferentes partes del mundo, sin embargo la mayor parte de la investigación se ha encaminado a la verificación de personas a través de línea telefónica [29]. Por la naturaleza de este proyecto resulta de interés describir los antecedentes que se tienen en materia de identificación y verificación, únicamente. 


\subsection{Esquema General de Verificación de Hablantes}

En general los sistemas de verificación actuales pueden describirse mediante cuatro módulos: adquisición, procesamiento, verificación y decisión.

Adquisición: En esta fase se consideran aspectos sobre el grabado de la voz. Actualmente se utilizan convertidores analógicos digitales que muestrean la voz en un intervalo de $8000 \mathrm{~Hz}$ a 20,000 $\mathrm{Hz}$ y con una resolución de 12 a 32 bits [17]. La frecuencia de muestreo es asignada de acuerdo a la calidad de voz a manejar. Para calidad telefónica se emplean $8000 \mathrm{~Hz}$. En la conformación de las bases de registros se consideran aspectos, principalmente, sobre la variabilidad de la voz y sobre otros, específicos del sistema de verificación.

Procesamiento: En esta fase se considera la extracción de características de las tramas de voz de los usuarios, que permitirán la operación del sistema de verificación. La extracción de vectores de características es realizada por medio del denominado análisis Cepstral. Una fase previa a dicho análisis puede consistir en la obtención de los coeficientes de predicción lineal (LPC, por sus siglas en inglés) [34], [17], ó bien la aplicación de un banco de filtros cubriendo el espectro en frecuencias de la señal [17]. Este análisis es también conocido como Mel-Warped Cepstrum, pues el banco de filtros es aplicado en una escala de frecuencias denominada Mel, la cual intenta emular el comportamiento fisiológico del oído humano [34].

Verificación: En esta fase se considera el proceso de generación del modelo correspondiente a cada hablante y la metodología para realizar la evaluación de los datos característicos a fin de determinar la mejor correspondencia con los modelos. Con este fin son empleados distintos procedimientos matemáticos. Algunos de los más referidos en los artículos de investigación son los Modelos de Mezclas Gaussianas (GMM, por sus siglas en inglés) [17] y los Modelos Ocultos de Markov (HMM, por sus siglas en inglés) [34]. También son reportados como procedimientos matemáticos las Máquinas de Soporte Vectorial (SVM, por sus siglas en inglés) [45], [54] y las redes neuronales artificiales [4].

Decisión: En esta fase se considera la aplicación de un modelo matemático que, con base en los resultados obtenidos, minimice la posibilidad de que suceda alguno de los dos errores conocidos: la aceptación de un solicitante inválido y el rechazo de un solicitante válido. La tabla 1.1 define, de manera lógica, ambos tipos de error; bajo el supuesto de que $\mathbf{H}=\{$ Muestra de voz de un usuario válido\}, $\neg H$ es la voz de un usuario no enrolado en el sistema y por tanto no válido. El sistema se limita a aceptar o rechazar la petición, entonces se tienen cuatro posibilidades de las cuales dos de ellas, $E_{1}$ y $E_{2}$, representan los dos tipos de error mencionados. 


\begin{tabular}{|c|c|c|}
\hline$\cdot$ & \multicolumn{2}{|c|}{ Acción del Sistema } \\
\hline Propuesta & Acepta & Rechaza \\
\hline $\mathbf{H}$ & $\mathbf{B}$ & $E_{1}$ \\
\hline$\neg H$ & $E_{2}$ & $\mathbf{B}$ \\
\hline
\end{tabular}

Tabla 1.1: Definición lógica de los errores posibles en un sistema de verificación.

El modelo para tratar con estos errores generalmente está basado en probabilidades y su objetivo es determinar un umbral de decisión. Esta fase está estrechamente ligada con la evaluación del desempeño del sistema. Actualmente se ha generalizado el uso de las curvas o gráficas DET (Detection Error Tradeoff), las cuales proporcionan un medio para comparar el desempeño de este tipo de sistemas.

La figura 1.1 muestra un esquema de un sistema de verificación general. En él pueden identificarse los cuatro módulos descritos previamente: adquisición, procesamiento, verificación y decisión.

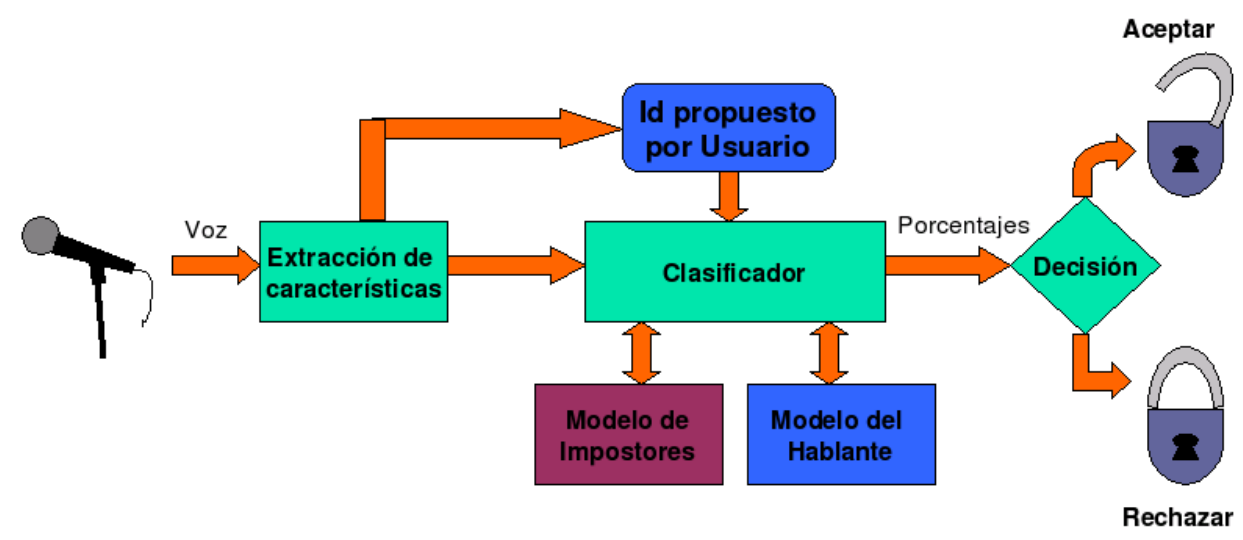

Figura 1.1: Esquema general de un sistema de verificación de hablantes.

\subsection{Estado del Arte}

En la tabla 1.2 se muestra el avance cronológico que se ha dado en la investigación e implementación de sistemas de reconocimiento automático de hablantes [34]. En este tabla se muestra que la verificación y la identificación pueden implementarse con o sin dependencia de frases específicas ( "text-dependent" y "text-independent", respectivamente), a su vez, dichas frases pueden tipificarse como provenientes de usuarios dispuestos o voluntarios o de 
usuarios indispuestos o involuntarios. En el error reportado se identifica si el valor corresponde a pruebas de verificación (v), o a pruebas de identificación (i), así como la duración de las frases utilizadas para el reconocimiento.

A pesar de que la información presentada en la tabla 1.2 no es suficiente para identificar características particulares sobre cada una de las implementaciones, como por ejemplo la(s) metodología(s) de prueba realizadas tanto en la verificación como en la identificación, es posible establecer una tendencia, la cual consiste en la mejoría en la precisión de los sistemas, a coste de un incremento en el tiempo de las tramas de voz usadas, ésto conjuntamente con un incremento en las dimensiones de las bases de voz empleadas, aspecto que puede considerarse natural, dado el desarrollo que a la par se ha suscitado en las tecnologías de la información. Aún con esta mejoría en el desempeño, la existencia de un porcentaje de error, obliga al uso de sistemas auxiliares adjuntos en aplicaciones donde se requiere o desea una mayor confiabilidad. Dichos sistemas auxiliares pueden consistir, por ejemplo, en tarjetas con información estadística particular que defina a cada usuario del sistema de reconocimiento [34]. En la práctica, se sabe que existe siempre la posibilidad de error en cualquier tarea de reconocimiento, sin embargo se desea minimizarla.

Aunque el primer registro reportado en la tabla 1.2 corresponde a 1974, se sabe que los primeros esfuerzos por realizar sistemas automáticos de reconocimiento fueron hechos a principios de los años 1950s. A continuación se da una breve historia sobre el reconocimiento por voz desde las primeras décadas.

En 1952 en los Laboratorios Bell, los científicos Davis, Biddulph y Balashek [37] construyeron una máquina para separar dígitos de secuencias de voz proporcionadas por un solo hablante. Este sistema consistía en medir las frecuencias de resonancia en la región vocal de cada dígito pronunciado para así discriminarlos en las frases pronunciadas. Posterior a ello y de forma independiente, los científicos Olson y Belar [26] en los Laboratorios RCA intentaron reconocer 10 sílabas distintas de un único hablante, en 10 palabras monosilábicas pronunciadas por el mismo. Este sistema, desarrollado en 1956, también se basó en la obtención de las características espectrales de la voz en regiones vocales y no vocales. En 1959, en el University College del Reino Unido, los científicos Fry y Denes [15] trataron de desarrollar un sistema de reconocimiento de fonemas para cuatro sonidos vocales y nueve consonantes. Para ello usaron un analizador de espectros y un verificador de patrones como sistema de decisión. Lo novedoso de este sistema fue el uso de información estadística de cada uno de los fonemas involucrados a fin de reconocerlos en las palabras pronunciadas por los hablantes, las cuales consistían de más de un fonema.

En este mismo periodo, en los Laboratorios Lincoln del MIT, fue desarrollado un sistema de reconocimiento de vocales. Este sistema fue hecho por Forgie y Forgie en 1959 


\begin{tabular}{|c|c|c|c|c|c|c|c|}
\hline AUTOR(ES) & ORG. & PROCESAMIENTO & METODOLOGÍA & CALIDAD & TEXTO & PERSONAS & ERROR \\
\hline Atal, 1974. [6] & $\mathrm{AT} \& \mathrm{~T}$ & Cepstrum & $\begin{array}{l}\text { Reconocimiento de } \\
\text { Patrones }\end{array}$ & Laboratorio & Dependiente & 10 & $\begin{array}{l}\mathrm{i}: 2 \%, \quad 0.5 \mathrm{~s} \\
\mathrm{v}: 2 \%, 1.0 \mathrm{~s}\end{array}$ \\
\hline $\begin{array}{l}\text { Markel, Davis, } \\
\text { 1974. [32] }\end{array}$ & STI & LP & $\begin{array}{l}\text { Estadísticas de largo } \\
\text { plazo }\end{array}$ & Laboratorio & Independiente & 17 & $\mathrm{i}: 2 \%, 39.0 \mathrm{~s}$ \\
\hline $\begin{array}{l}\text { Furui, } \\
{[44]}\end{array}$ & $\mathrm{AT} \& \mathrm{~T}$ & $\begin{array}{l}\text { Cepstrum Normali- } \\
\text { zado }\end{array}$ & $\begin{array}{l}\text { Reconocimiento de } \\
\text { Patrones }\end{array}$ & Teléfono & Dependiente & 10 & $\mathrm{v}: 0.2 \%, 3.0 \mathrm{~s}$ \\
\hline $\begin{array}{l}\text { Schwartz, et. } \\
\text { al., 1982. [43] }\end{array}$ & BBN & LAR & PDF no paramétrico & Teléfono & Independiente & 21 & $\mathrm{i}: 2.5 \%, 2.0 \mathrm{~s}$ \\
\hline $\begin{array}{l}\mathrm{Li} \text { and Wrench, } \\
\text { 1983. [38] }\end{array}$ & ITT & LP y Cepstrum & $\begin{array}{l}\text { Reconocimiento de } \\
\text { Patrones }\end{array}$ & Laboratorio & Independiente & 11 & $\begin{array}{l}\text { i: } 21 \% . \quad 3.0 \mathrm{~s} \\
\text { i: } 4 \%, 10.0 \mathrm{~s}\end{array}$ \\
\hline $\begin{array}{l}\text { Doddington, } \\
\text { 1985. [21] }\end{array}$ & TI & Filter Bank & $\begin{array}{l}\text { Discrete Time Warp- } \\
\text { ping }\end{array}$ & Laboratorio & Dependiente & 200 & i: $0.8 \%, 6.0 \mathrm{~s}$ \\
\hline $\begin{array}{l}\text { Soong, et. al., } \\
\text { 1985. [20] }\end{array}$ & $\mathrm{AT} \& \mathrm{~T}$ & LP & $\begin{array}{l}\text { VQ, Likelihood Ratio } \\
\text { Distortion }\end{array}$ & Laboratorio & $\begin{array}{l}10 \quad \text { Dígitos } \\
\text { Individuales }\end{array}$ & 100 & $\begin{array}{l}\text { i: } 5.0 \%, \quad 1.5 \mathrm{~s} \\
\text { i: } 1.5 \%, 3.5 \mathrm{~s}\end{array}$ \\
\hline $\begin{array}{l}\text { Higgins, Wohl- } \\
\text { ford, 1986. [3] }\end{array}$ & ITT & Cepstrum & $\begin{array}{l}\text { DTW } \\
\text { Scoring }\end{array}$ & Laboratorio & Independiente & 11 & $\begin{array}{l}\mathrm{v}: 10.0 \%, 2.0 \mathrm{~s} \\
\mathrm{v}: 4.5 \%, 10 \mathrm{~s}\end{array}$ \\
\hline $\begin{array}{l}\text { Attilli, et. al., } \\
\text { 1988. }[27]\end{array}$ & RPI & $\begin{array}{l}\text { Cepstrum, LP, Auto- } \\
\text { corr. }\end{array}$ & $\begin{array}{l}\text { Estadísticas a Largo } \\
\text { Plazo }\end{array}$ & Laboratorio & Dependiente & 90 & $\mathrm{v}: 1.0 \%, 3.0 \mathrm{~s}$ \\
\hline $\begin{array}{l}\text { Higgins, et. al., } \\
\text { 1991. [1] }\end{array}$ & ITT & LAR, LP, Cepstrum & $\begin{array}{l}\text { DTW } \\
\text { Scoring }\end{array}$ & Oficina & Dependiente & 186 & $\mathrm{v}: 1.7 \%, 10.0 \mathrm{~s}$ \\
\hline $\begin{array}{l}\text { Tishby, } 1991 . \\
{[41]}\end{array}$ & $\mathrm{AT} \& \mathrm{~T}$ & LP & HMM (AR mix) & Teléfono & $\begin{array}{l}10 \text { Dígitos in- } \\
\text { dividuales }\end{array}$ & 100 & $\begin{array}{l}\text { v: } 2.8 \%, 1.5 \mathrm{~s} \\
\text { v: } 0.8 \%, 3.5 \mathrm{~s}\end{array}$ \\
\hline $\begin{array}{l}\text { Reynolds, } \\
1995 . \\
\text { [12],Reynolds, } \\
\text { Carlson, } 1995 . \\
{[13]}\end{array}$ & $\begin{array}{l}\text { MIT- } \\
\text { LL }\end{array}$ & Mel-Cepstrum & HMM-GMM & Oficina & Dependiente & 138 & $\begin{array}{l}\text { i: } 0.8 \%, 10.0 \mathrm{~s} \\
\text { v: } 0.12 \%, 10 \mathrm{~s}\end{array}$ \\
\hline $\begin{array}{l}\text { Che, Lin, } 1995 . \\
\text { [7] }\end{array}$ & Rutgers & Cepstrum & HMM & Oficina & Dependiente & 138 & $\begin{array}{l}\mathrm{i}: 0.56 \%, 2.5 \mathrm{~s} \\
\text { i: } 0.14 \%, 10 \mathrm{~s} \\
\text { v:0.62\%, } 2.5 \mathrm{~s}\end{array}$ \\
\hline $\begin{array}{l}\text { Colombi, et. } \\
\text { al., 1996. [28] }\end{array}$ & AFIT & $\begin{array}{l}\text { Cep, Eng dCep, dd- } \\
\text { Cep }\end{array}$ & HMM monófono & Oficina & Dependiente & 138 & $\begin{array}{l}\text { i: } 0.22 \%, \\
10.0 \mathrm{~s} \\
\text { v: } 0.28 \%, 10 \mathrm{~s}\end{array}$ \\
\hline $\begin{array}{l}\text { Reynolds, } \\
\text { 1996. [11] }\end{array}$ & $\begin{array}{l}\text { MIT- } \\
\text { LL }\end{array}$ & $\begin{array}{l}\text { Mel-Cepstrum , Mel- } \\
\text { dCepstrum }\end{array}$ & HMM, GMM & Teléfono & Independiente & 416 & $\begin{array}{l}\text { v: } 16.0 \%, \quad 3 \mathrm{~s} \\
\text { v: } 8.0 \%, \quad 16 \mathrm{~s} \\
\text { v: } 5 \%, 30 \mathrm{~s}\end{array}$ \\
\hline $\begin{array}{l}\text { J.P. Campbell, } \\
\text { 1997. [34] }\end{array}$ & JHU & LPCC, LAR, LSPF & DTW & Teléfono & Dependiente & YOHO, 138 & $\mathrm{v}: 0.5 \%$ EER \\
\hline $\begin{array}{l}\text { X. Dong, et. } \\
\text { al., } 2001[56]\end{array}$ & $\begin{array}{l}\text { DFEEC- } \\
\text { UEN }\end{array}$ & LPC & GMM, SVM & Teléfono & Independiente & ҮОНО, 138 & $\mathrm{v}: 0.5 \%$ EER \\
\hline $\begin{array}{l}\text { H. Flengei, et. } \\
\text { al., } 2002[23]\end{array}$ & $\begin{array}{l}\text { DIZ- } \\
\text { UZ }\end{array}$ & $\begin{array}{l}\text { MFCC, } \\
\text { Clustering }\end{array}$ & SVM & Teléfono & Independiente & OGI, 60 & v:3.1\% EER \\
\hline $\begin{array}{l}\text { W.M. Camp- } \\
\text { bell, et. al., } \\
2004 \text { [55] }\end{array}$ & $\begin{array}{l}\text { MIT- } \\
\text { LL }\end{array}$ & $\begin{array}{l}\text { Phone and word se- } \\
\text { quences }\end{array}$ & SVM & Teléfono & Independiente & $\begin{array}{l}\text { NIST- } \\
2003,739\end{array}$ & v: $2.5 \%$ EER \\
\hline $\begin{array}{l}\text { V. Wan, et. al., } \\
2005 \text { [51] }\end{array}$ & $\begin{array}{l}\text { DCS- } \\
\text { US }\end{array}$ & $\begin{array}{l}\text { Sequence Discrimina- } \\
\text { tion, PLPCC }\end{array}$ & $\begin{array}{l}\text { SVM, Score Space } \\
\text { Kernels }\end{array}$ & Teléfono & Independiente & PolyVar, 38 & v: $4.0 \%$ EER \\
\hline $\begin{array}{l}\text { S. Raghavan, } \\
\text { et. al. } 2006 \\
{[45]}\end{array}$ & $\mathrm{MSU}$ & $\mathrm{MFCCs}$ & SVM-RBF & $\longrightarrow$ & Independiente & NIST-2003 & $\begin{array}{ll}\operatorname{Min} & \mathrm{DCF} \\
0.1406 & \end{array}$ \\
\hline $\begin{array}{l}\text { W.M. Camp- } \\
\text { bell, et. al., } \\
2006[54]\end{array}$ & $\begin{array}{l}\text { MIT- } \\
\text { LL }\end{array}$ & $\mathrm{MFCC}$ & $\begin{array}{l}\text { GMM supervectors, } \\
\text { SVM }\end{array}$ & Teléfono & Independiente & NIST-2005 & $\begin{array}{l}\text { v: } 3.77 \% \\
\text { EER }\end{array}$ \\
\hline $\begin{array}{l}\text { K. Daoudi, et. } \\
\text { al., 2007. [36] }\end{array}$ & $\begin{array}{l}\text { IRIT- } \\
\text { CNRS }\end{array}$ & $\mathrm{LFCC}$ & SVM-PoS & - & Independiente & $\begin{array}{l}\text { NIST-SRE } \\
2003,2004\end{array}$ & $\begin{array}{l}\min _{0.0469} \\
\text { DCF }\end{array}$ \\
\hline
\end{tabular}

Tabla 1.2: Cronología del desarrollo de aplicaciones en reconocimiento de personas por voz. 
[35] y consistió en el reconocimiento de 10 vocales incrustadas en frases con el formato $\backslash b \backslash$-vocal- $\backslash t \backslash$, de forma independiente al hablante considerado. Nuevamente fue utilizado un analizador basado en un banco espectral de filtros y en una estimación, variable en el tiempo, para reconocer la vocal pronunciada. Posteriormente, en los años sesentas, fueron introducidas diferentes ideas inicialmente por laboratorios de origen japones; una de las primeras fue presentada por Suzuky y Nakata del Radio Research Lab en Tokyo [31], la cual consistió en un sistema de reconocimiento de vocales implementado en hardware; éste contaba con un banco de filtros como analizador de espectro y la salida de cada filtro era conectada (y multiplicada por un peso asociado) a un circuito lógico complejo que funcionaba como sistema de decisión de vocales. Un segundo ejemplo es el sistema desarrollado en la Universidad de Kyoto por Sakai y Doshita en 1962 [47]. La implementación fue en hardware y consistió de un segmentador de voz junto con un analizador de cruces por cero (zero-crossing), el cual detectaba las secuencias de la trama correspondientes a voz.

En esta misma década (1960s) se iniciaron tres proyectos que tuvieron efectos importantes en el desarrollo de sistemas de reconocimiento [10]:

- El primer proyecto fue iniciado por Martin conjuntamente con los Laboratorios RCA[48], para proporcionar soluciones a problemas relacionados con la no uniformidad de las escalas temporales, en secuencias de voz. En este proyecto se desarrollaron diferentes métodos de normalización en la escala tiempo, basados en la detección de inicio y fin de segmentos de voz, de forma que fue posible reducir la variabilidad en los porcentajes de reconocimiento para segmentos de voz fonéticamente equivalentes. Martin, por último, desarrolló un método adecuado y fundó una de las primeras compañías (Threshold Technology) que desarrollaron productos comercialmente aptos basados en reconocimiento por voz.

- Casi a la par del proyecto anterior, en la Unión Soviética, Vintsyuk [49] propuso una metodología basada en programación dinámica para alinear en el tiempo dos pronunciaciones del mismo hablante. Este trabajo manejó conceptos sobre la metodología que hoy se conoce como Dynamic Time Warping (DTW), así como algoritmos rudimentarios para reconocimiento de palabras. Su trabajo permaneció desconocido hasta principios de los noventas, cuando fue estudiado y empleado por otros investigadores.

- El último proyecto considerado en esta década fue iniciado por Reddy en 1966 [16], mismo que fue pionero en el campo que hoy se conoce como Reconocimiento Continuo de Voz (Continuous Speech Recognition), el cual se basa en el reconocimiento dinámico de fonemas. Este proyecto dio inicio a uno posterior en la Universidad Carnegie Mellon. Las primeras demostraciones de este último se dieron en dicha universidad en 1973; el sistema llamado Hersay I, era capaz de recibir información hablada y hacer una interpretación semántica para determinar la instrucción correspondiente, seleccionándola 
de los movimientos posibles de las piezas en el juego de ajedrez. La conclusión fundamental fue que es posible emplear información sintáctica, semántica y contextual de la voz, para reducir el número de opciones posibles a ser consideradas en un sistema que entienda, aunque en forma limitada, el lenguaje humano.

En los años 1970s se cuentan múltiples ejemplos de desarrollos, algunos de ellos mostrados en la tabla 1.2, los cuales permitieron la conformación de áreas de estudio. De esta manera es posible caracterizar esta década a partir del área de investigación o metodología(s) empleada(s) en los proyectos de investigación [10]:

- En el área de Reconocimiento de Palabras (Isolated-Word Recognition) o Reconocimiento Discreto de Voz (Discrete-Utterance Recognition), se tienen referencias como la proporcionada por Velichko y Zagoruyco en la Unión Soviética [52]. Sus estudios proporcionaron ideas acerca del uso del Reconocimiento de Patrones en Reconocimiento por Voz. Las investigaciones de Sakoe y Chiba [25] en Japón proporcionaron información sobre como usar de forma exitosa, los métodos de programación dinámica para reconocimiento de hablantes. Por su parte Itakura en los Estados Unidos de Norteamérica, mostró como las ideas de codificación de voz mediante LPC, pueden ampliarse al reconocimiento por voz mediante el uso de una métrica basada en estos parámetros espectrales [18].

- En el área de Dictado Automático de Vocabulario Extenso (Large Vocabulary Automatic Speech Dictation), se tienen referencias del trabajo desarrollado en la IBM, en donde los investigadores se centraron en tres tareas principales: el llamado Lenguaje Raleigh para consultas simples a bases de datos [8], el Lenguaje Laser de Texto para Patentes usado para transcribir patentes mediante Laser [19] y el Sistema de Tareas de Oficina usado para dictado de cartas simples [19].

- Como una división del área anterior, se generaron investigaciones en lo que fueron los Laboratorios AT\&T Bell. Los investigadores realizaron una serie de experimentos con el objetivo de hacer sistemas de reconocimiento de voz completamente independientes del hablante para aplicaciones en telecomunicaciones [39], particularmente servicios automáticos a distancia mediante la conversación entre una máquina y un hablante. Con este fin se realizaron diferentes algoritmos para tratar con la gran variabilidad de las palabras y expresiones de una extensa población. Los primeros resultados de esta investigación se obtuvieron una década después y proporcionaron diferentes modelos para la voz.

En la década de 1980s se identifican las siguientes características principales [10]:

- Prevaleció un enfoque hacia el reconocimiento de palabras conjuntas o frases (connected word recognition), a diferencia de la década anterior en donde el enfoque principal fue 
el reconocimiento de palabras y fonemas de forma individual. Así el objetivo de esta nueva generación de investigaciones fue el reconocimiento de palabras concatenadas o cadenas de palabras pronunciadas de forma continua o fluida, con base en patrones de palabras concatenadas. Una aplicación de estas investigaciones fue la desarrollada por la compañía Nippon Electric Corporation, donde Sakoe [24] programó un sistema dinámico de dos niveles para lograr el reconocimiento de frases pronunciadas de forma fluida.

- La metodología en las investigaciones se caracterizó por un cambio en la tecnología al pasar de un enfoque basado en plantillas o patrones de referencia a métodos de modelado basado en estadísticas. Entre los principales métodos puede identificarse los Modelos Ocultos de Markov (Hidden Markov Models, HMM).

Aún cuando la diversidad de aplicaciones e investigaciones se incrementó en la década pasada (1990s) es posible mencionar los siguientes aspectos:

- Los laboratorios de la DARPA (Defense Advanced Research Projects Agency), continuaron con los proyectos iniciados en la década pasada relacionados con reconocimiento de vocabulario extenso y con sistemas de reconocimiento de secuencias continuas de voz [10]. También dando énfasis a la recuperación de información hablada de sistemas en vuelo y a la transcripción de noticias o mensajes transmitidos.

- El uso de la tecnología de reconocimiento por voz se incrementó dentro de redes de telecomunicación tradicionales (teléfono) para automatizar y mejorar la operación de servicios [5].

- Se intensificó el uso de Modelos Ocultos de Markov como metodología en los sistemas de reconocimiento [7], [28], [41]. Adicionalmente se inicia el uso de Modelos de Mezclas Gaussianas (Gaussian Mixture Models, GMM) como una metodología basada en estadísticas [11], [12], [13].

A pesar de que la identificación es el caso más general y enriquecedor en el ámbito científico, comparado con la verificación, la tendencia en las aplicaciones comerciales en la última década (2000s) es la siguiente [17]:

- La mayoría de los sistemas comerciales consiste en verificación por voz.

- Estos sistemas están basados en esquemas donde se tienen usuarios dispuestos o voluntarios, es decir que desean ser identificados por el sistema.

- Son dependientes de texto; se pronuncian frases pequeñas proporcionadas por el sistema. Generalmente las claves de acceso son dígitos individuales. 
- Siguen usando las principales metodologías de la década pasada. Adicionalmente se proponen nuevas metodologías como las Máquinas de Soporte Vectorial [23], [45], [51], [53], [55], [56] (Support Vector Machines, SVM) y Redes Neuronales Artificiales [42] (Artificial Neural Networks, ANN).

Existen diferentes compañías que han desarrollado aplicaciones comerciales de sistemas de reconocimiento [34]: ITT, Lernout \& Hauspie, T-NETIX, Veritel y Voice Control Systems. El objetivo principal de sus aplicaciones se orienta al resguardo de seguridad en controles de acceso, banco por teléfono y manejo de tarjetas de crédito vía telefónica, con el objetivo de reducir transacciones fraudulentas.

La tendencia en el uso de la voz como característica biométrica en sistemas de reconocimiento y el que éstos se realicen con calidad telefónica reside, principalmente, en los siguientes hechos [17]:

- En muchas regiones el teléfono alámbrico es el único o principal medio de comunicación, por lo que el reconocimiento por voz resulta natural como sistema de seguridad.

- Los sistemas telefónicos constituyen un sistema que provee una red que, además de ser popular, tiende a ser ubícuita y desde la cual se pueden obtener las muestras de voz.

- Debido a los puntos anteriores, no existe necesidad de un procesamiento adicional en la captura de las muestras, a diferencia de sistemas de reconocimiento basados en otras características biométricas, como las huellas digitales o la cara.

- En general, el costo y tipo de la infraestructura requerida para la implementación es reducido y accesible.

- Se cuenta actualmente con una experiencia en esta área en investigación, desarrollo y evaluaciones, de más de 50 años, la cual incluye aplicaciones comerciales.

Las restricciones aplicadas a estos sistemas, generalmente con la finalidad de incrementar la precisión, en algunos casos pueden volverlos inflexibles a modificaciones o alteraciones, por ejemplo en el ambiente de operación o para usuarios no dispuestos o involuntarios. Para estos casos la verificación por voz independiente de texto es más adecuada.

A pesar de la amplia experiencia acumulada en el reconocimiento de hablantes, no es inmediato determinar alguna dirección clara en el futuro de las investigaciones. Sin embargo existen retos que pueden orientarlas, como son la pérdida de consistencia de los sistemas frente a variaciones en el canal y otras condiciones de error, como por ejemplo en el micrófono o la existencia de un SNR (Signal to Noise Ratio) bajo. 
Muchos de los sistemas basan su operación en características acústicas de la voz (por ejemplo su espectro en frecuencia), por lo que las variaciones en el canal influyen considerablemente en el desempeño global. Un desacoplamiento o rompimiento de dicha dependencia, en alguna forma aún no considerada, haría posible una consistencia más robusta en las aplicaciones [17]. Adicionalmente existen otros niveles en el sistema de audición humano, que no han sido explorados o que se están empezando a explorar, por ejemplo la habilidad del oído humano para reconocer voces que le son familiares. Esta perspectiva motiva la posibilidad de emular y aprovechar hábitos del habla de largo plazo en los sistemas automáticos.Las líneas de investigación que apuntan hacia estos tópicos han emergido recientemente y quizá requieran de algunas de las técnicas ya desarrolladas o incluso de su fusión o combinación. 


\section{Capítulo 2}

\section{Sistema Automático de Verificación}

En este capítulo se presenta la aplicación de una metodología para la creación de un sistema automático de verificación de personas a partir de la voz, basado en la teoría de Máquinas de Soporte Vectorial como sistema de clasificación.

El proceso de verificación requiere, como parte esencial, la creación de módelos correspondientes a los usuarios considerados como válidos e inválidos. Esta tarea se basa en la hipótesis fundamental de que es posible asociar de forma biunívoca registros de voz de cada hablante con el mismo. Cuando se logra satisfacer esta hipótesis en alguna implementación, se cuenta entonces con un procedimiento de asociación biunívoca. De esta manera, dada una muestra de voz y a través del mismo procedimiento, es posible determinar si ésta corresponde o no al usuario que solicita ser verificado. Es entonces necesario conformar un modelo para cada voz y establecer así un procedimiento de asociación. En las siguientes secciones se exponen los planteamientos al respecto.

\subsection{Metodología}

En la figura 2.1 se muestra un esquema que plantea la metodología utilizada en este trabajo. Los etapas son implementadas de izquierda a derecha y de arriba hacia abajo. Dicho esquema incluye la comprobación del sistema con voz en tiempo real.

La metodología propuesta consiste en conformar la base de registros de voz para una número fijo de personas. A partir de ésta es posible obtener los vectores de características para cada hablante. Dicho conjunto de vectores permitirá definir los conjuntos de entrenamiento y de prueba. El conjunto de entrenamiento será usado por el sistema de soporte vectorial para conformar el modelo correspondiente a cada hablante. La formación de modelos es determinante en la creación del sistema automático. Una vez construidos los modelos 


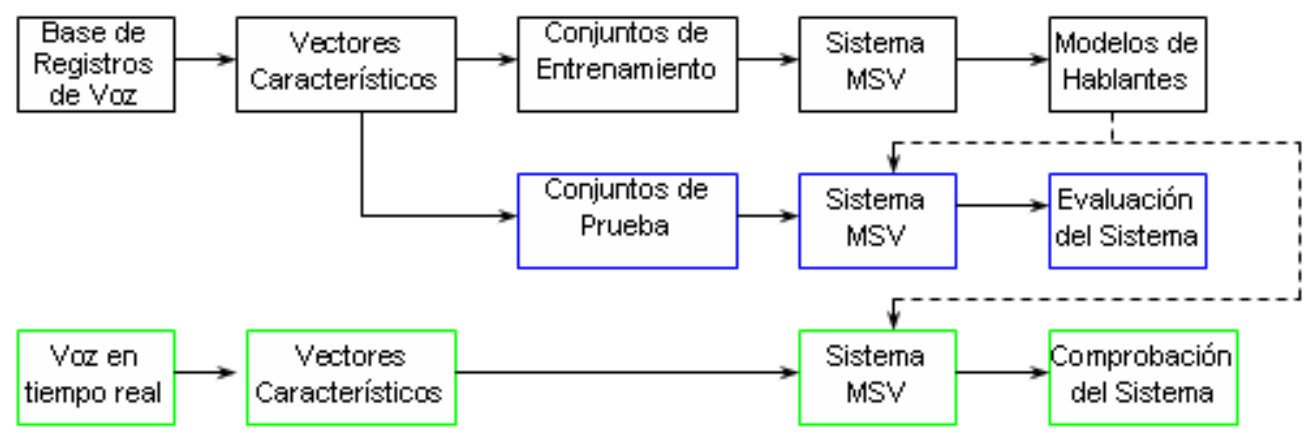

Figura 2.1: Metodología para implementar un sistema automático de verificación mediante Máquinas de Soporte Vectorial.Las flechas continuas muestran el orden de aplicación de cada etapa. Las flechas punteadas muestran la dependencia de ciertas etapas a los modelos creados.

de los hablantes, se aplicarán los conjuntos de prueba para obtener una evaluación del desempeño del sistema implementado.

A fin de comprobar la operación se realizarán pruebas con voz en tiempo real. Para ello la voz será grabada desde un micrófono, sometida a la extracción de vectores de características y después ingresada al sistema con soporte vectorial, para determinar la operación ante solicitudes válidas e inválidas.

\subsection{Base de Registros de Voz}

La creación de una base de registros de voz tiene como principal objetivo contar con muestras representativas de voz de cada una de las personas que participarán en el sistema, sea como usuarios válidos (enrolados) o como usuarios no válidos. En este último caso, las muestras de voz proporcionadas por los participantes permitirán formar, aunque no totalmente, el modelo de los usuarios no válidos, conocido comunmente como complemento o background. Se debe considerar que para un usuario, denotado por $U_{i}$, el resto de los usuarios enrolados también servirá para conformar el background. La tabla 2.1 establece la notación que será válida a partir de aquí. 


\begin{tabular}{|l|l|}
\hline Notación & Significado \\
\hline$U_{i}$ & Un hablante que participa con su voz en el sistema de verificación. \\
\hline$\left\{U_{j}\right\}_{j=1}^{N}$ & $\begin{array}{l}\text { Conjunto que denota a todos los usuarios o hablantes (válidos e inválidos) } \\
\text { involucrados en el sistema, referido como universo de usuarios. En este } \\
\text { caso el total de usuarios es denotado por } N .\end{array}$ \\
\hline$\left\{U_{i}\right\}^{c}$ & $\begin{array}{l}\text { Conjunto que denota al complemento de usuarios a } U_{i} \text { respecto del uni- } \\
\text { verso de usuarios. }\end{array}$ \\
\hline$S_{i}$ & Muestra de voz del usuario $i$. \\
\hline$\left\{x_{i k}\right\}_{k=1}^{l}$ & Vectores de características del usuario $U_{i}$ \\
\hline$M_{i}$ & Modelo del usuario $i$. \\
\hline
\end{tabular}

Tabla 2.1: Notación utilizada para denotar los elementos del sistema de verificación.

Para la conformación de la base de registros de voz pueden identificarse dos puntos principales a considerar:

Representatividad. Los registros deben ser representativos por su contenido de información. Para ello se consideran algunos aspectos de la variabilidad de la voz en las escalas de tiempo y amplitud, así como el contenido de fonemas y sílabas de las frases pronunciadas.

Universalidad. La conformación de conjuntos complemento a un usario $\left(\left\{U_{i}\right\}^{c}\right)$ es únicamente respecto al conjunto total o universo de usuarios, es decir no incluye de forma real a todos los usuarios no válidos. La conformación de complementos para cada usuario será universal en la medida en la que se incluya a potenciales usuarios no válidos del sistema.

A pesar de que formalmente el lenguaje no es una característica intrínseca del ser humano, pues es adquirido de manera empírica a través del tiempo y con la adecuada socialización, si puede considerarse como una característica distintiva de la especie. La voz es una sucesión lógica de sonidos básicos o fundamentales llamados fonemas. Físicamente, dichos fonemas son ondas mecánicas esféricas con una representación matemática particular, la cual puede ser periódica. Cada lenguaje, entendido éste como un conjunto de símbolos que permiten la comunicación verbal (v.g. español, ingés, francés), posee un conjunto finito de fonemas y ciertas reglas de ordenamiento que determinan la lógica y semántica en las secuencias o sucesiones pronunciadas, las cuales pueden incluir intervalos de silencio. La creación de un sistema automático que determine si una persona es quien dice ser (verificación), se basa, como se ha mencionado, en la hipótesis de que las características biológicas y fisiológicas del sistema del habla son tales, que la voz generada posee propiedades cualitativas y cuantitativas que son únicas para cada hablante. Esta hipótesis fundamental es sin embargo empírica, pues 
no se tiene en el campo de la biometría referencias que demuestren la unicidad en alguna(s) propiedad(es) de la voz.

Aún si se supone una validación correcta de la hipótesis, la gran variabilidad en las propiedades cuantitativas y cualitativas de la voz, impide la comparación directa de fonemas, como técnica de reconocimiento de hablantes. En consecuencia es necesario considerar el tratamiento, análisis y comprobación de la(s) hipótesis propuesta(s) y la implementación de metodologías que traten con la variabilidad de la voz y con los factores que inciden en dicha variabilidad, a fin de reducir el porcentaje de error e incrementar el desempeño del proceso de verificación.

Idealmente la creación de un sistema de verificación universal por voz es deseable, sin embargo actualmente no es viable por razones de implementación. De esta manera la base de registros de voz deberá considerar tanto la variabilidad de la voz como la orientación o fin al que se destinará, con el objetivo de acotar sus alcances y con ello también sus características. Algunos de los aspectos específicos que pueden ser considerados para la conformación de una base de registros se enlistan a continuación [34], [30], [46]:

1. Sesiones de grabado. A fin de considerar la variabilidad de la voz en diferentes días, es posible realizar para cada persona, grabaciones de voz en dos sesiones con al menos cinco días de separación.

2. Velocidad de lectura. Consiste en el grabado de voz a la velocidad usual del hablante y también a mayor y menor velocidad de la usual. La variabilidad de la voz en la escala temporal, es una de las dificultades que se presenta en el reconocimiento de fonemas y palabras. Considerar este aspecto en el grabado de los registros, a pesar de ser un tanto subjetivo, amplía la utilidad de los mismos y su representatividad.

3. Frases de prueba. El objetivo de estas frases es contar con datos de prueba para verificar el comportamiento del sistema ante intrusos; en este caso se tienen frases particulares para cada hablante así como frases que pronuncian por igual todos los hablantes. Estas frases también permiten el uso de los registros de voz en sistemas que son dependientes e independientes del texto pronunciado.

4. Niveles de ruido. La presencia de ruido en el ambiente de grabación no favorece el proceso de verificación pues dificulta el procesamiento de la señal real. Bajo esta consideración, la grabación debiera realizarse en un ambiente controlado sin ninguna otra influencia audible diferente a la voz. Sin embargo, si el sistema será usado en un ambiente donde existe influencias de sonido externas (por ejemplo en una oficina), lo adecuado sería considerar dichos niveles de ruido en los registros de voz, pues las muestras que se ingresen al sistema lo incluirán. 
5. Edades y sexo de los hablantes. Se sabe que el intervalo de frecuencias audibles que conforman una señal de voz, está determinada en parte, por el sexo del hablante, por lo que es de importancia adquirir registros de ambos sexos a fin de considerar la variabilidad genérica de la voz. No existe entonces restricción para la participación de hombres y mujeres a menos que el sistema, por la naturaleza de su uso, lo requiera. Por otro lado se sabe que la voz es sensible a la edad en un mismo hablante y que la mayor variabilidad de ésta sucede en la adolescencia, por ello debe identificarse y considerarse de forma adecuada la población que estará involucrada en el sistema de reconocimiento, para garantizar la estabilidad temporal en el desempeño del mismo.

6. Número de hablantes. Se considera que cada hablante genera un registro de voz. El número de hablantes determinará directamente la información almacenada. En teoría, dada la hipótesis de unicicidad de la voz, el incrementar el número de registros no incrementa la complejidad de la operación de un sistema de reconocimiento. En realidad el número de registros es un factor importante para conformar un sistema confiable, ya que incrementa la probabilidad de encontrar correlación entre los registros de voz.

7. Sistema de grabado. Es posible considerar la influencia del sistema de grabado en los datos obtenidos, para ello pueden utilizarse micrófonos de diferente calidad para realizar grabaciones simultáneas o en sesiones diferidas. Este aspecto agrega un factor que permite adecuar los registros de voz a diferentes sistemas de grabado. También permite analizar la influencia de estos medios en los sistemas de reconocimiento.

8. Espontaneidad en la voz. Este aspecto considera diferencias en la pronunciación entre voz por lectura de texto y por pronunciación espontánea. Si se considera que los sistemas de reconocimiento pueden operar solicitando la pronunciación de un texto leído o la repetición de una frase asignada, deben considerarse ambos tipos de grabación.

9. Variación del canal. Es posible variar la distancia entre el micrófono y el hablante a fin de considerar la influencia de variaciones en el canal de transmisión, lo cual puede representar una condición real de operación del sistema de reconocimiento.

10. Distribución de fonemas y sílabas. Una muestra de voz es representativa en la medida en la que incluye los fonemas y sílabas del idioma en la que se obtiene. Las frases pronunciadas deben tener una distribución de fonemas y sílabas que corresponde con la distribución usual del idioma.

\subsubsection{Protocolo de Grabación}

Con base en los aspectos planteados previamente es posible establecer un protocolo de grabación, el cual permite uniformizar la adquisición de muestras de voz de cada uno de los participantes. Las tareas que integran este protocolo se describen a continuación: 
a) Pronunciación del nombre del hablante. Esta tarea permite la identificación de los registros de voz y proporciona una frase particular para cada usuario la cual le es familiar y permite la grabación de una muestra de voz con espontaneidad.

b) Pronunciación de los dígitos del 0 al 9. La pronunciación solicitada al hablante inicia con el número cero y hasta el nueve. De esta manera se genera una primera frase equivalente para todos los hablantes así como el registro de pronunciación individual de los 10 dígitos de forma espontánea.

c) Pronunciación individual de dos cadenas de dígitos. Se generaron de forma aleatoria las cadenas de dígitos 4, 2, 3, 1, 7 y 8, 6, 0, 1, 5, las cuales son pronunciadas con una pausa intermedia entre ambas por todos los participantes. Esta tarea porporciona la pronunciación de dígitos en desorden y de forma individual lo cual permite contar con información sobre posibles diferencias con respecto a la pronunciación en forma ordenada, también proporciona una frase general obtenida por lectura.

d) Repetición de una cadena de dígitos. Se genera de forma aleatoria y para cada hablante una cadena de 5 dígitos (ver Tabla 2.2). La cadena es repetida dos veces con una pausa intemedia entre cada una. Esta tarea proporciona información sobre la repetición de cadenas de dígitos leídos individualmente y sobre posibles similitudes o diferencias en la pronunciación.

e) Pronunciación de frases de texto particulares. Se seleccionaron para cada hablante, dos frases de texto de diferentes fuentes, considerando aquellas que tuvieran alrededor de 15 palabras y sin considerar a priori su contenido fonético o silábico (ver Tabla 2.2). Esta tarea proporciona frases leídas de texto y particulares para cada hablante, las cuales pueden ser consideradas como aleatorias.

f) Pronunciación de una misma frase de texto. Todos los participantes leyeron la siguiente frase:

Fue Platón quien dio su estructura dialéctica, mucho más amplia y sutil, al nuevo género literario, aportándole un espíritu mucho más audaz y una claridad artística que anteriormente no tenía. Es por ello que está considerado el más grande de los prosistas helénicos; sus diálogos escritos alcanzaron la madurez y perfección como paradigma clásico de las discusiones filosóficas a cielo abierto.

Esta frase proporciona información representiva de la pronunciación de cada hablante, de los diferentes fonemas y sílabas que conforman el español. También trata con la pronunciación leída de texto, equivalente para todos los participantes.

g) Pronunciación de texto con diferente rapidez. Los participantes leyeron el texto de la tarea f) con mayor y posteriormente con menor rapidez de lo que usualmente lo hacen. Con ello se cuenta con información representativa sobre posibles modificaciones de la 
voz ante circunstancias como el nerviosismo o la calma que favorecen la presencia de errores.

Las siete tareas descritas previamente, conforman el protocolo de grabación que fue usado para obtener cada uno de los registros de voz $S_{j}$ los cuales generan la base de registros denominada Voces-MCyTI. Algunas de las características consideradas para la obtención de dichas muestras son las siguientes:

1. Las sesiones de grabación fueron realizadas en una oficina de la UAM Iztapalapa, a puerta cerrada, pero sin mayor cuidado respecto a la presencia de fuentes de ruido externas.

2. Después de cada una de las tareas del protocolo, se realizó una pausa y una reanudación de la grabación. En las tareas de lectura se solicitó al participante se familiarizara con la frase a pronunciar antes de realizar la grabación.

3. Los días y horarios de grabación fueron dispersos, dependiendo de la disposición de los participantes.

4. El micrófono para las grabaciones fue de calidad media. Las muestras $S_{j}$ fueron obtenidas a una frecuencia de muestreo de $8000 \mathrm{~Hz}$ considerando el uso del sistema a través de línea telefónica. No hubo control estricto respecto de la distancia entre el hablante y el micrófono.

5. La distribución de edades de los 17 participantes se ilustra en la figura 2.2.

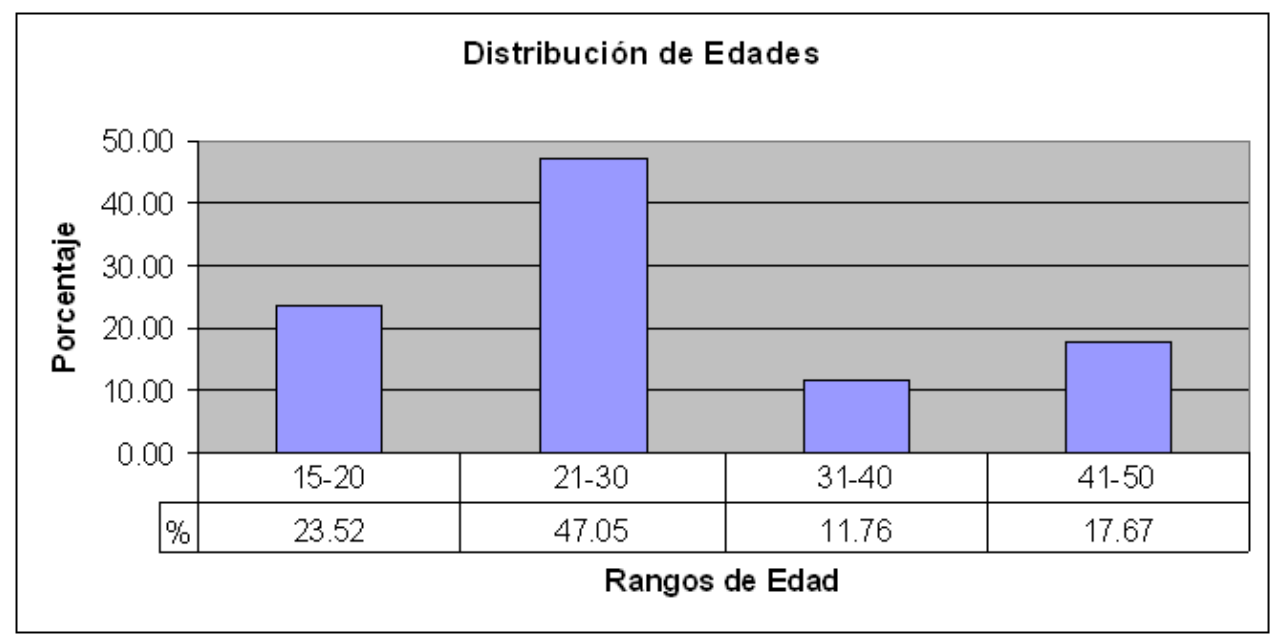

Figura 2.2: Distribución de edades de los participantes en la base Voces-MCyTI.

En las tablas 2.2 y 2.3 se muestran algunas de las frases particulares pronunciadas por los participantes así como la referencia al archivo de sonido generado. 


\begin{tabular}{|c|c|c|c|}
\hline Archivo & Tarea d) & Tarea e) & $\begin{array}{l}\text { Fecha de } \\
\text { grabación }\end{array}$ \\
\hline mcyti_1.wav & $4,6,2,3,9$ & $\begin{array}{l}\text { 1) Alemania abre sus puertas al mundo con la duda de } \\
\text { qué tan lejos llegará su selección. 2) Venderán cerveza } \\
\text { en los estadios. Para los teutones tomar esta bebida } \\
\text { es casi una religión. }\end{array}$ & $23 / 05 / 2006$ \\
\hline mcyti_2.wav & $3,2,9,7,1$ & $\begin{array}{l}\text { 1) La única que te da el doble de puntos del progra- } \\
\text { ma de recompensas vida bancomer. } 2 \text { ) Cada veintemil } \\
\text { puntos acumulados puedes canjearlos por un boleto de } \\
\text { avión, viaje redondo, a cualquier parte del país. }\end{array}$ & $15 / 06 / 2006$ \\
\hline mcyti_3.wav & $7,1,3,6,2$ & $\begin{array}{l}\text { 1) La danzonera dimas fue fundada en } 1921 \text { por Ama- } \\
\text { dor Pérez Torres mejor conocido como Dimas. 2) } \\
\text { Chávez, lector de poesía, precisó que con esta reedición } \\
\text { rinde homenaje a López Velarde y a Fermín Revueltas. }\end{array}$ & $19 / 10 / 2006$ \\
\hline mcyti_4.wav & $5,9,4,8,0$ & $\begin{array}{l}\text { 1) El caifán Oscar Chávez cantará danzonetes, sus } \\
\text { clásicas por tí y la niña de Guatemala, en su concierto. } \\
\text { 2) Chávez agregó: Todo lo que ha generado mi disco } \\
\text { Chiapas es para apoyar a las comunidades indígenas. }\end{array}$ & $18 / 10 / 2006$ \\
\hline mcyti_5.wav & $8,5,0,2,1$ & $\begin{array}{l}\text { 1) Erasmo Capilla nació en Jalapa, estudió música con } \\
\text { su padre y después en la Universidad Veracruzana. 2) } \\
\text { A los } 14 \text { años de edad ganó el Concurso Nacional de } \\
\text { Violín para la Juventud Mexicana. }\end{array}$ & $18 / 10 / 2006$ \\
\hline mcyti_6.wav & $4,6,9,7,3$ & $\begin{array}{l}\text { 1) Para desmantelar el amarillismo, la producción de } \\
\text { Vuelo } 93 \text { se ha cuidado en documentar la historia. } 2 \text { ) } \\
\text { Asimismo, los actores fueron escogidos en virtud a su } \\
\text { semejanza física con las personas reales. }\end{array}$ & $18 / 10 / 2006$ \\
\hline mcyti_7.wav & $6,7,9,5,4$ & $\begin{array}{l}\text { 1) La hipertensión arterial y el hábito de fumar favo- } \\
\text { recen la muerte de millones de personas. 2) Reducir el } \\
\text { consumo de grasas, incrementar la ingesta de frutas y } \\
\text { verduras y modificar hábitos de vida. }\end{array}$ & $18 / 10 / 2006$ \\
\hline mcyti_8.wav & $2,0,8,3,1$ & $\begin{array}{l}\text { 1) A veces la ciencia olvida que los hombres son el } \\
\text { otro componente vital del proceso reproductivo. 2) De } \\
\text { los trecemil ochocientos sesenta embarazos reportados } \\
\text { mil quinientos seis se interrumpieron. }\end{array}$ & $19 / 10 / 2006$ \\
\hline mcyti_9.wav & $1,3,7,6,0$ & $\begin{array}{l}\text { 1) En su más reciente película Munich, el primer pa- } \\
\text { lestino asesinado por los servicios secretos. 2) En la } \\
\text { inexorable realidad de Gaza no hay Aladino ni lámpa- } \\
\text { ra maravillosa que valgan. }\end{array}$ & $19 / 10 / 2006$ \\
\hline
\end{tabular}

Tabla 2.2: Frases particulares pronunciadas por los participantes en la base Voces-MCyTI. 


\begin{tabular}{|c|c|c|c|}
\hline Archivo & Tarea d) & Tarea e) & $\begin{array}{l}\text { Fecha de } \\
\text { grabación }\end{array}$ \\
\hline mcyti_10.wav & $2,9,5,4,8$ & $\begin{array}{l}\text { 1) La historia era increíble, en efecto, pero se impuso a } \\
\text { todos, porque sustancialmente era cierta. 2) Verdadero } \\
\text { era también el ultraje que había padecido; sólo eran } \\
\text { falsas las circunstancias. }\end{array}$ & $23 / 10 / 2006$ \\
\hline mcyti_11.wav & $1,4,9,3,6$ & $\begin{array}{l}\text { 1) Nació en el sudeste de Asia, no es muy alta y de } \\
\text { color verde pálido, más conocida como noni. 2) Las } \\
\text { últimas investigaciones indican que la planta está re- } \\
\text { pleta de químicos que revierten el cancer. }\end{array}$ & $20 / 10 / 2006$ \\
\hline mcyti_12.wav & $8,7,1,0,4$ & $\begin{array}{l}\text { 1) Un indígena asiste en Sucre, Bolivia, a la presenta- } \\
\text { ción del programa Microsoft Windows en Quechua. 2) } \\
\text { Evo Morales anunciará la nacionalización de las con- } \\
\text { cesiones para la exportación de las riquezas naturales. }\end{array}$ & $25 / 10 / 2006$ \\
\hline mcyti_13.wav & $3,6,5,9,2$ & $\begin{array}{l}\text { 1) En la opuesta margen resplandecía, bajo el último } \\
\text { sol o bajo el primero, la evidente Ciudad de los In- } \\
\text { mortales. 2) Esta ciudad es tan horrible que su mera } \\
\text { existencia y perduración contamina el presente y el } \\
\text { porvenir. }\end{array}$ & $26 / 10 / 2006$ \\
\hline mcyti_14.wav & $5,3,1,0,8$ & $\begin{array}{l}\text { 1) La muerte hace preciosos y patéticos a los hombres; } \\
\text { todo es irrecuperable y azaroso. 2) Entre los inmorta- } \\
\text { les, en cambio, cada acto y cada pensamiento es el eco } \\
\text { de otros que en el pasado lo antecedieron. }\end{array}$ & $23 / 10 / 2006$ \\
\hline mcyti_15.wav & $4,9,2,7,6$ & $\begin{array}{l}\text { 1) La mujer quiere resistir, pero dos hombres la han } \\
\text { tomado del brazo y la hechan sobre Otálora. 2) Que le } \\
\text { han permitido el amor, el mando y el triunfo, porque } \\
\text { ya lo daban por muerto. }\end{array}$ & $26 / 10 / 2006$ \\
\hline mcyti_16.wav & $0,2,7,1,9$ & $\begin{array}{l}\text { 1) Le encontraron la misma sustancia pocos días des- } \\
\text { pués de haberse proclamado campeon del Tour de } \\
\text { Francia. 2) El entrenador Trevor denunció el uso de } \\
\text { esteroides THG, entre deportistas de Estados Unidos. }\end{array}$ & $07 / 11 / 2006$ \\
\hline mcyti_17.wav & $3,6,0,4,9$ & $\begin{array}{l}\text { 1) Básteme recordar que el desertor malhirió o mató a } \\
\text { varios de los hombres de cruz. 2) No iba a consentir } \\
\text { el delito de que se matara a un valiente y se puso a } \\
\text { pelear contra los soldados. }\end{array}$ & $16 / 11 / 2006$ \\
\hline
\end{tabular}

Tabla 2.3: Frases particulares pronunciadas por los participantes en la base Voces-MCyTI. 


\subsubsection{Análisis Fonológico y Silábico}

A continuación se presenta el análisis fonológico y silábico de la frase pronunciada en las tareas f) y g) del protocolo de grabación utilizado. El análisis consisitió en separar por fonemas y sílabas dicha frase y obtener la distribución de frecuencias correspondiente. Esta distribución es la que corresponde con la usual del español [30], criterio que determinó la selección de la misma.

La distribución de fonemas se realizó considerando un total de 28, con base en la notación SAMPA (Speech Assesment Methods Phonetics Alphabet) para el español (ver Apéndice A.1). Así, la frase consta de un total de 318 fonemas y su distribución se encuentra resumida en la tabla 2.4 y esquematizada en la figura 2.3 .

\begin{tabular}{|c|c|c|c|c|c|c|c|}
\hline $\mathbf{a}$ & $12.89 \%$ & $\mathbf{e}$ & $10.37 \%$ & $\mathbf{o}$ & $9.43 \%$ & $\mathbf{s}$ & $8.49 \%$ \\
\hline $\mathbf{i}$ & $8.49 \%$ & $\mathbf{n}$ & $5.03 \%$ & $\mathbf{t}$ & $5.34 \%$ & $\mathbf{d} / \mathbf{D}$ & $4.72 \%$ \\
\hline $\mathbf{l}$ & $5.66 \%$ & $\mathbf{k}$ & $5.34 \%$ & $\mathbf{u}$ & $4.71 \%$ & $\mathbf{r}$ & $4.71 \%$ \\
\hline $\mathbf{m}$ & $3.14 \%$ & $\mathbf{p}$ & $2.51 \%$ & $\mathbf{T}$ & $1.88 \%$ & $\mathbf{b} / \mathbf{B}$ & $0.63 \%$ \\
\hline $\mathbf{r r}$ & $2.20 \%$ & $\mathbf{G}$ & $0.63 \%$ & $\mathbf{x}$ & $0.63 \%$ & $\mathbf{f}$ & $1.26 \%$ \\
\hline $\mathbf{j}$ & $0.94 \%$ & $\mathbf{t S}$ & $0.63 \%$ & $\mathbf{L}$ & $0.31 \%$ & $\mathbf{j} \mathbf{j} / \mathbf{J} / \mathbf{w}$ & $0 \%$ \\
\hline
\end{tabular}

Tabla 2.4: Distribución de fonemas en la frase de las tareas f) y g) del protocolo de grabación.

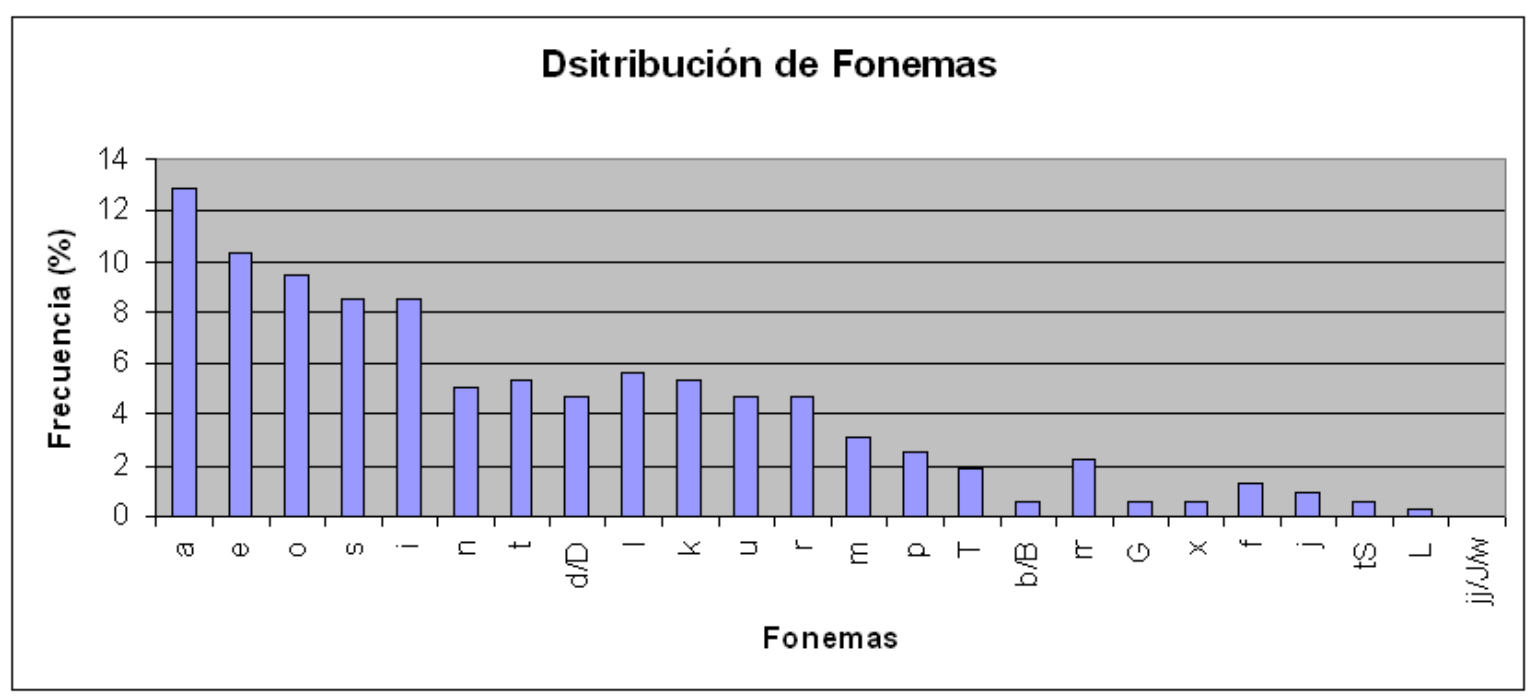

Figura 2.3: Gráfica de la distribución de fonemas en la frase de las tareas f) y g) del protocolo de grabación. 
La distribución de sílabas se obtuvo considerando 8 tipos. Con base en ésto la frase considerada consta de un total de 136 sílabas y la distribución de éstas se encuentra resumida en la tabla 2.5 y esquematizada en la figura 2.4.

\begin{tabular}{|c|c|c|}
\hline CLAVE & DESCRIPCIÓN & PORCENTAJE \\
\hline CV & Consonante-Vocal & $46.32 \%$ \\
\hline CVC & Consonante-Vocal-Consonante & $22.79 \%$ \\
\hline V & Vocal & $6.61 \%$ \\
\hline VC & Vocal-Consonante & $8.08 \%$ \\
\hline CVV & Consonante-Vocal-Vocal & $6.61 \%$ \\
\hline CCV & Consonante-Consonante-Vocal & $3.67 \%$ \\
\hline O & Otras sílabas & $5.88 \%$ \\
\hline
\end{tabular}

Tabla 2.5: Distribución de sílabas de la frase de las tareas f) y g) del protocolo de grabación.

La finalidad de este análisis es contar con una frase balanceada que contenga la información correspondiente a los fonémas y sílabas que pueden ser pronunciados en el español y que sea así representativa del lenguaje.

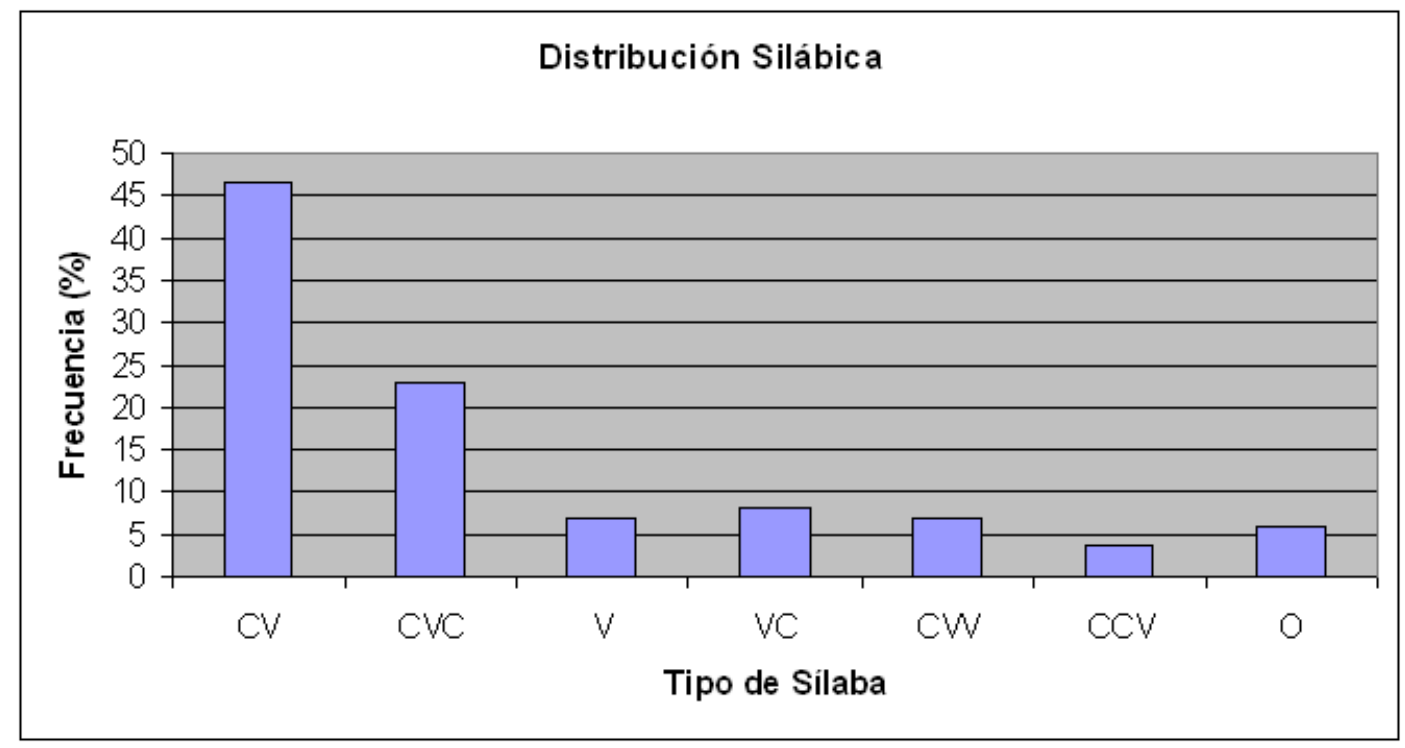

Figura 2.4: Gráfica de la distribución de sílabas de la frase de las tareas f) y g) del protocolo de grabación. 


\subsection{Procesamiento de la Voz}

En la sección 1.3 (Estado del Arte) se hizo mención de las diferentes técnicas y procedimientos que pueden ser implementados para que, dada una señal de voz se obtengan valores numéricos que la caractericen o representen. En general, los objetivos principales de dicha representación son:

- Sintetizar la información contenida en la señal de voz, de manera que se reduzca el número de datos o valores que la representan.

- Evitar el manejo de información poco significativa, es decir los datos que dificultan las tareas de reconocimiento, como el ruido o los intervalos entre palabras (silencios).

- Minimizar el manejo de información redundante contenida en la señal de voz, por ejemplo los fonemas pronunciados.

- Facilitar la definición de un modelo que corresponda de manera única a la voz considerada.

- Proporcionar los datos de entrada, simplificar la operación y en su caso mejorar la efectividad de algún sistema de clasificación o verificación automática.

A continuación se describe brevemente el procedimiento que, en particular, se propone para la realización de este trabajo, mismo que se basa en el análisis de la voz por su contenido frecuencial. Por su importancia en el procesamiento de la voz, ciertos módulos serán descritos detalladamente en secciones posteriores. La figura 2.5 muestra la secuencia de módulos a los que se somete la señal de voz, $S(n)$, hasta obtener los vectores de características, $x(m)$.

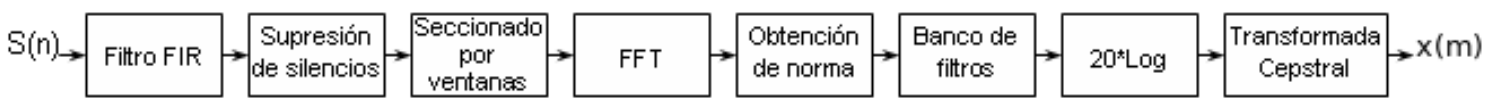

Figura 2.5: Esquema del procesamiento de voz para extracción de características

El procedimiento consiste en la obtención de los llamados coeficientes MFCC ( Mel Frecuency Cepstral Coeficients), los cuales constituyen los vectores de características de la voz, con los que se pretende cumplir los objetivos establecidos previamente. Su uso es propuesto y descrito en diferentes artículos de investigación que han sido publicados recientemente y en los cuales se presenta como una técnica exitosa en el reconocimiento de hablantes [17], [36], [46].

1. Filtro FIR: La señal de voz es sometida a un filtro con respuesta al impulso finita. Este es un filtro pasa banda entre los $300 \mathrm{~Hz}$ y $3300 \mathrm{~Hz}$, de orden 51, simétrico. Para 
mejorar la resolución espectral (debido al truncamiento temporal de la señal), se aplica una ventana de Hamming antes del filtrado. La finalidad del filtro es eliminar las frecuencias correspondientes al ruido y obtener una mejora en el SNR.

2. Supresión de silencios: Se implementa un procedimiento para suprimir de la señal los intervalos entre palabras los cuales corresponden al ruido en el ambiente. Este procedimiento consiste en analizar el comportamiento de la potencia promedio de la señal en ventanas de longitud $M$.

3. Seccionado por ventanas: El seccionado o separación de la señal por ventanas es la preparación para aplicar la transformada de Fourier a la señal. El seccionado o segmentado de la señal se realiza en ventanas de tiempo donde el proceso es considerado estacionario. Para la voz un intervalo típico es de 20 a 30 ms. También se considera un traslape entre ventanas consecutivas de $10 \mathrm{~ms}$.

4. FFT: Se aplica la transformada rápida de Fourier (Fast Fourier Transform) a cada una de las ventanas consideradas. Esta transformación puede ser realizada mediante diferentes algoritmos y usando una cantidad de puntos $N$ variable. Generalmente $N$ es una potencia de 2 y es más grande que el número de muestras $M$ de las ventanas. Usualmente se usan 512 puntos.

5. Obtención de norma: De manera análoga a la transformada de Fourier continua, su similar discreta está en el plano complejo, por lo que es necesario obtener la norma o módulo de cada uno de los puntos obtenidos. El resultado de esta operación conforma una representación gráfica simétrica, por lo que sólo es necesario considerar la mitad de los puntos obtenidos por cada ventana analizada.

6. Banco de filtros: Con el fin de obtener una representación de la envolvente de cada uno de los espectros resultantes, se aplica a cada uno un banco de filtros. Este es una serie de filtros pasabanda que son multiplicados uno a uno con el espectro dado y promediados los resultados correspondientes. De esta manera se reduce nuevamente la longitud de los vectores pudiendo, dado un filtro, enfatizar el comportamiento del espectro en una frecuencia particular. El banco es definido por la forma de los filtros y la escala de frecuencias a la que son ubicados; frecuencia inicial, frecuencia media y frecuencia final. Los filtros pueden ser de forma triangular y estar ubicados de acuerdo a la escala de frecuencias de Mel, obtenida a partir de la siguiente ecuación [17]:

$$
f_{M E L}=1000 \log \left(1+\frac{f_{\text {lin }}}{1000}\right) / \log (2)
$$

7. Transformación $20^{*} \log$ : En esta parte se obtiene el logaritmo de cada uno de los coeficientes obtenidos y se multiplica por 20 para obtener valores en decibeles (dB). Los datos obtenidos son llamados vectores espectrales (denotados por $V$ ). 
8. Transformada Cepstral [46]: La última transformada aplicada a la señal, sobre cada uno de los vectores espectrales, consiste en obtener a partir de éstos, los coeficientes cepstrales, los cuales están definidos por la siguiente ecuación:

$$
x(n)=\sum_{k=1}^{K} V_{k} \cos \left(\frac{n \pi}{K}\left(k-\frac{1}{2}\right)\right), n=1,2, \ldots, L .
$$

donde $V_{k}$ son los elementos de un vector espectral y $K$ es la longitud de éste. $L$ es el número de coeficientes cepstrales que se desean calcular $(L \leq K)$.

Con respecto a los parámetros involucrados en la extracción de vectores de características, se tienen valores típicos empleados y con los que se han obtenido resultados favorables [40]. Los parámetros iniciales utilizados en el procesamiento de los registros de voz $S_{j}$ son resumidos en la tabla 2.6 .

\begin{tabular}{|c|c|}
\hline Parámetro & Valor \\
\hline Banda del filtro FIR & $300 \mathrm{~Hz}-3300 \mathrm{~Hz}$ \\
\hline Orden del filtro FIR & 51, Hamming \\
\hline Tiempo de ventana $(M)$ & $30 \mathrm{~ms}(240$ muestras $)$ \\
\hline Tiempo de traslape & $10 \mathrm{~ms}(80$ muestras $)$ \\
\hline Orden de FFT $(N)$ & 512 puntos \\
\hline Orden del Filtro Mel $(K)$ & 31 \\
\hline Orden del Vector Cepstral $(L)$ & 13 \\
\hline
\end{tabular}

Tabla 2.6: Parámetros iniciales usados para el procesamiento de los registros de voz $S_{j}$ y la obtención de los vectores de características $x_{j}(n)$.

\subsubsection{Supresión de Silencios}

Una señal de ruido puede definirse como cualquier influencia externa o diferente de nuestra señal objetivo, en este caso la voz. Cuando se realiza la grabación de la voz, tanto la influencia del ambiente como la del propio sistema de grabación, alteran la señal, modificando los niveles reales con los que originalmente es emitida, es decir se produce una distorsión. Los efectos totales correspondientes a un sistema de grabación en un ambiente dado, pueden ser obtenidos grabando dicho ambiente. La señal obtenida considera el ruido del sistema y puede así ser analizada para determinar sus características. Cuando la grabación incluye la voz de un hablante, los niveles de ruido permanecen y se superponen a la señal de voz, de manera que la señal resultante es una suma directa de ambas. 
Bajo estas condiciones se propone una metodología para determinar en dicha señal los intervalos correspondientes al habla. Dos aspectos importantes que justifican tener un procedimiento como éste son:

- La posibilidad de reducir el tamaño de los registros de voz, previo a cualquier procesamiento de los mismos, lo cual disminuiría el tiempo de ejecución del sistema.

- Omitir de los registros de voz, información que no corresponde a los hablantes y que, de permanecer, agregaría correlación entre los conjuntos de vectores de características, provocando mayor dificultad en el proceso de clasificación.

La supresión de intervalos que no corresponden a voz, parte de la hipótesis de que es posible discriminar a partir de la intensidad de un punto de la señal grabada, si éste corresponde a voz o a ruido. Si suponemos que $S_{j}(n)$ representa una grabación, puede considerarse que es medida como una diferencia de potencial $E$ (Volts). Entonces $S_{j}^{2}(n)$ es proporcional a la potencia de la señal:

$$
P=E . I=E \frac{E}{R}=\frac{1}{R} E^{2} .
$$

Al graficar $S_{j}(n)$ y $S_{j}^{2}(n)$ para algunas muestras, se observa que el comportamiento en la magnitud de esta última contrasta en mejor forma los periodos correspondientes a voz de los de ruido. De esta forma se usa $S_{j}^{2}(n)$ en lugar de $S_{j}(n)$ y se considera como la potencia de la señal. El procedimiento que se propone para realizar la discriminación de puntos correspondientes a ruido de los de voz se describe a continuación.

1. Se asumen dos hipótesis necesarias: a) Se considera que existen condiciones de grabación invariantes en cuanto a los niveles de ruido presentes en el sistema. Lo anterior significa que no existen fuentes ni sumideros de ruido en el tiempo de grabación de $S_{j}$. b) Cada uno de los registros $S_{j}$ que sean sometidos al proceso de supresión de silencios, deben tener un intervalo de silencio o pausa del hablante de $r_{j}$ segundos $\left(r_{j}>0\right)$ al inicio de la trama. La figura 2.6 muestra un segmento inicial del registro $S_{1}$.

2. Se obtiene la potencia promedio por segmentos de la señal con base en la siguiente ecuación:

$$
P_{j}(n)_{\text {prom }}=\frac{1}{M} \sum_{k=n}^{n+M} S_{j}^{2}(k),
$$

es decir se obtiene el promedio de la potencia de la señal en un segmento a partir de la muestra $n$ y hasta la muestra $n+M$. La figura 2.7 muestra un segmento inicial de la gráfica de $S_{1}^{2}(n)$ mientras que la figura 2.8 permite observar el efecto de la ecuación 2.4 para la misma muestra en el mismo segmento. 


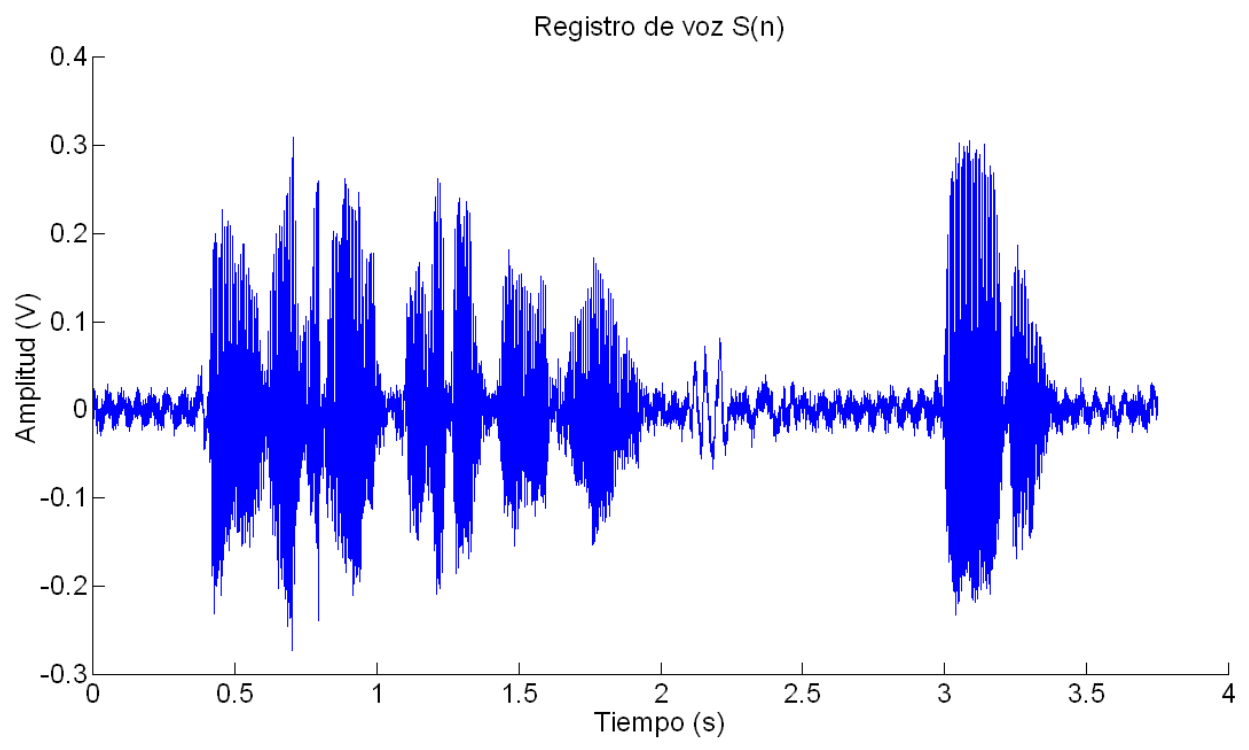

Figura 2.6: Segmento inicial del registro $S_{1}$.

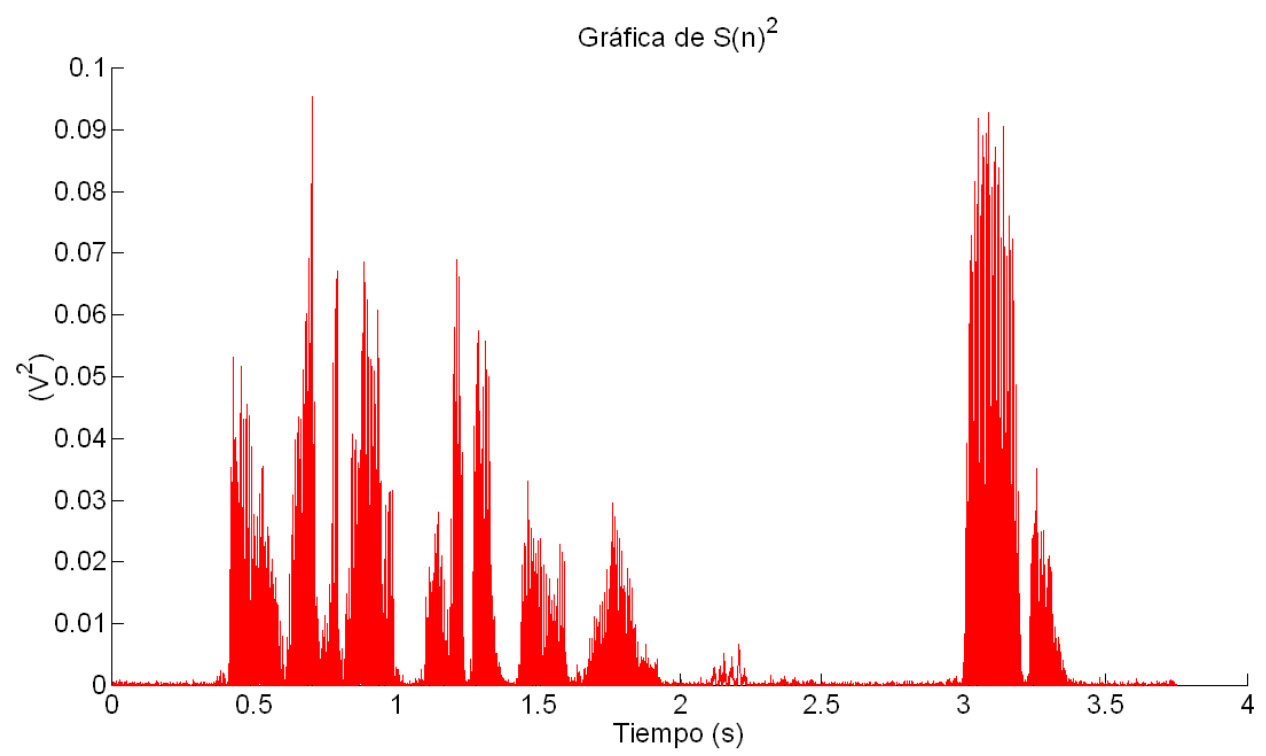

Figura 2.7: Esquema de $S_{1}^{2}(n)$. 


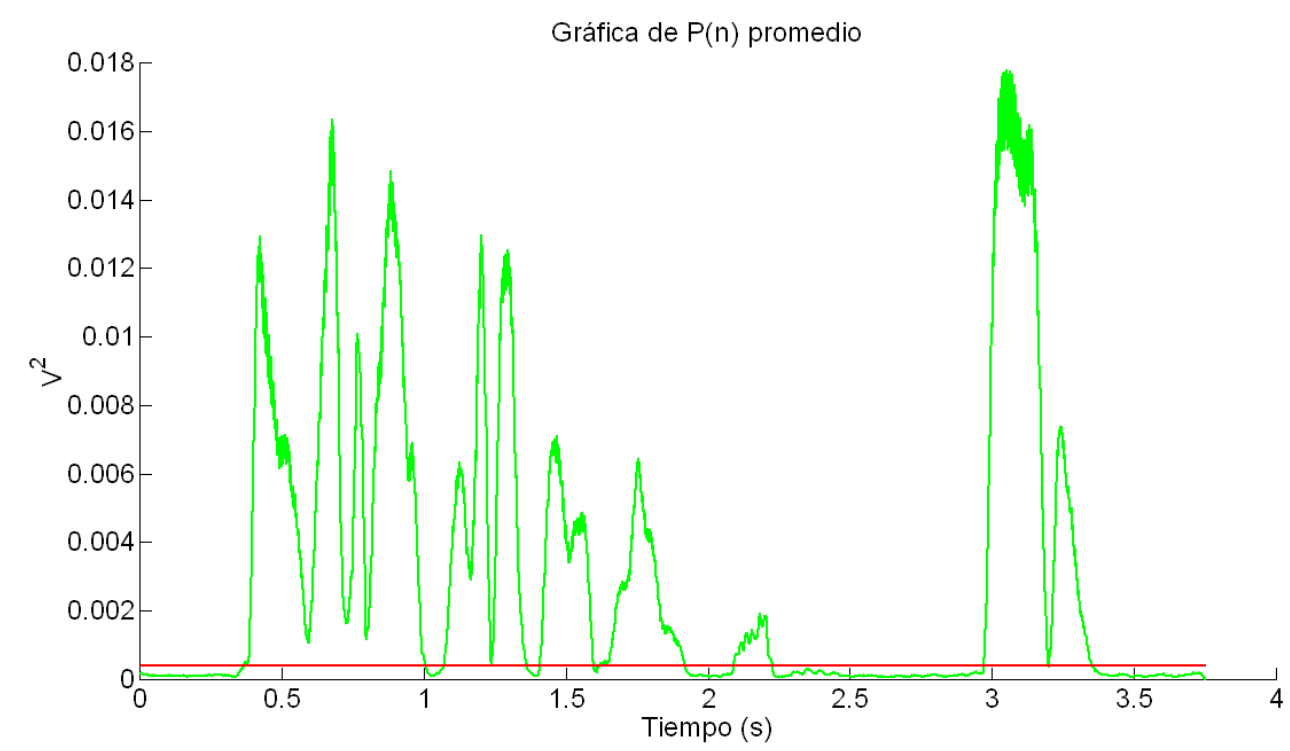

Figura 2.8: Gráfica de $P_{1}(n)_{\text {prom }}$ y nivel de umbral $U_{1}$.

3. Con base en $P_{j}(n)_{\text {prom }}$ y dado un valor de umbral $u_{j}$, se filtra $S_{j}(n)$ mediante la siguiente función:

$$
S_{j}(n)_{v o z}= \begin{cases}S_{j}(n) & \text { si } P_{j}(n)_{\text {prom }} \geq u_{j} \\ 0 & \text { si } P_{j}(n)_{\text {prom }}<u_{j}\end{cases}
$$

La señal $S_{j}(n)_{v o z}$ permite discriminar los intervalos de voz de las pausas hechas por el hablante y que corresponden al ruido del sistema. Para ello es suficiente con eliminar de $S_{j}(n)_{v o z}$ los puntos de la señal para los que $P_{j}(n)_{\text {prom }}<u_{j}$.

4. Con base en las hipótesis del punto 1 , se puede establecer que los $r_{j}$ segundos iniciales de cada señal $S_{j}$ corresponden a una muestra del ruido total del sistema y que esta muestra es estadísticamente equivalente a cualquiera otra de la señal, de longitud $r_{j}$ y que corresponda también a ruido. Por lo tanto parámetros como el promedio, la varianza y/o la desviación estandar de la muestra, deben ser equivalentes o aproximados para cualquier muestra de ruido, considerada en las mismas condiciones. Por lo anterior el nivel de umbral $u_{j}$ que proponemos se establece de la siguiente forma:

$$
u_{j}=\frac{1}{r_{j} f_{s}} \sum_{k=1}^{r_{j} f_{s}} S_{j}^{2}(k)+c_{j} \sqrt{\sigma_{S_{j}}^{2}},
$$

donde $f_{s}$ es la frecuencia de muestreo, $\sigma_{S_{j}}^{2}$ está dada por 


$$
\sigma_{S_{j}}^{2}=E\left[\left(S_{j}^{2}(n)-\frac{1}{r_{j} f_{s}} \sum_{k=1}^{r_{j} f_{s}} S_{j}^{2}(k)\right)^{2}\right]
$$

y $c_{j}>1$ es una constante que incrementa el valor de umbral ajustándolo para cada registro $S_{j}$.

En la figura 2.9 se esquematiza el comportamiento del procedimiento de supresión de silencios para un segmento inicial del registro de voz $S_{1}$.

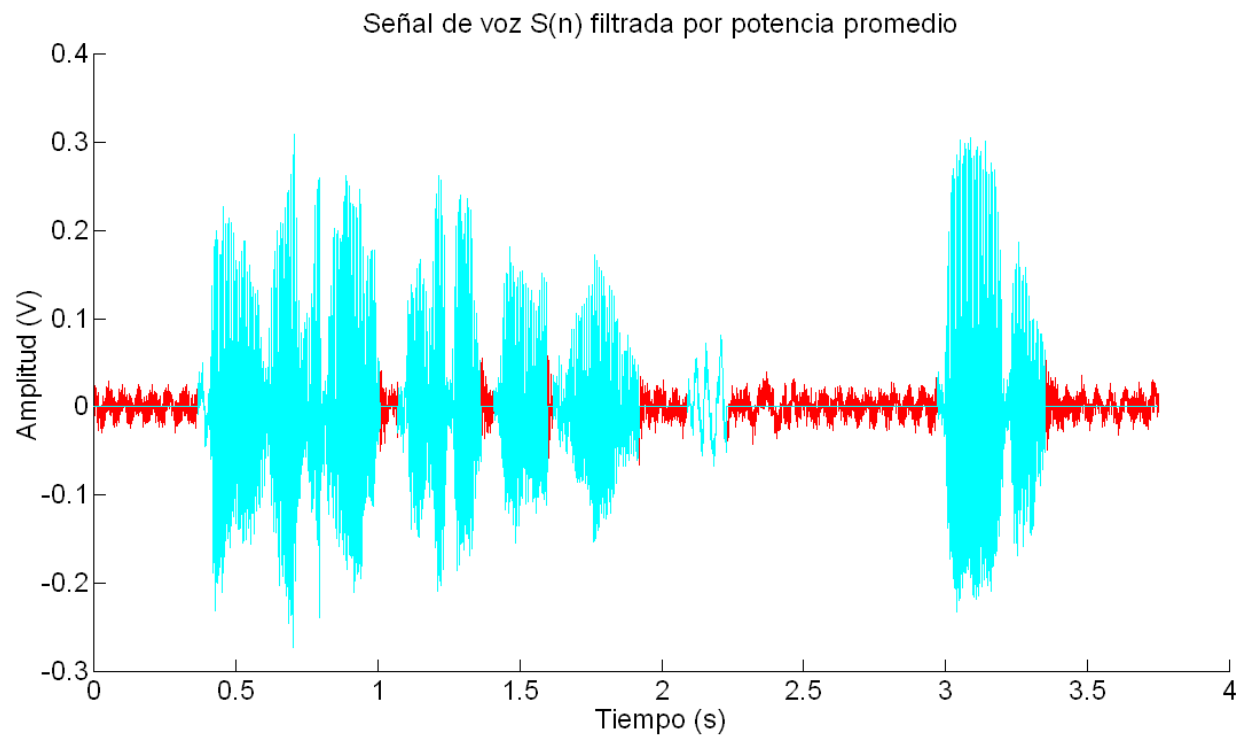

Figura 2.9: Señal con supresión de silencios $S_{1}(n)_{v o z}$ superpuesta a la señal original.

Este procedimiento está basado en la medición de las propiedades de una muestra representativa de ruido, la cual se toma del inicio del registro $S_{j}$. La efectividad de la función 2.5, para establecer las pausas o intervalos de silencio de un hablante, está determinada por el valor de umbral $u_{j}$, ya que éste discrimina los puntos que deben omitirse de la señal, con base en el comportamiento promedio de la potencia en un segmento. Lo anterior implica que al incrementarse, en promedio, la potencia del nivel de ruido con respecto a la potencia promedio de la voz, la supresión de silencios puede eliminar también segmentos de voz. Un parámetro que permite medir esta relación es la llamada razón señal a ruido (Signal to Noise Ratio, SNR) definida en la siguiente ecuación [33]:

$$
S N R=10 \log \left(\frac{P_{\text {señal }}}{P_{\text {ruido }}}\right) \text {, en } d B
$$


En consecuencia es deseable incrementar el valor del $S N R$ en los registros de voz para mejorar la efectividad de la supresión de silencios. Debido a ésto el filtro FIR tiene prioridad respecto de la etapa de supresión en el proceso de extracción de los vectores de características.

\subsubsection{Banco de Filtros Mel}

La aplicación de la transformada de Fourier discreta a los registros de voz, permite obtener información sobre el contenido frecuencial de la misma por cada ventana o segmento analizado. Sin embargo la cantidad de información obtenida es excesiva y puede complicar la clasificación o separación de vectores. Se da entonces la necesidad de sintetizar la información contenida en el espectro de frecuencias de una forma discreta. La aplicación de un banco de filtros al espectro frecuencial obedece al planteamiento dado previamente.

Como se ha mencionado la elección de un banco de filtros en la escala Mel tiene fundamento en que ésta emula en mejor forma, la resolución con la que el oído humano funciona. Esto significa que enfatiza el contenido en ciertos intervalos de frecuencias, de forma análoga al comportamiento fisiológico del oído humano.

A continuación se describe la conformación y aplicación de este banco de filtros en el procesamiento y extracción de características de la voz [17].

1. Se establece una frecuencia máxima de aplicación del banco de filtros. En este caso $f_{\max }=3300 \mathrm{~Hz}$, debido a que el filtro FIR aplicado en etapas previas, limita el contenido frecuencial de los registros de voz $S_{j}$ a dicho valor.

2. Con base en $f_{\max }$ y la dimensión del banco de filtros, dada por $K$, se obtiene un intervalo $\Delta$ de construcción del banco en una escala lineal de frecuencia:

$$
\Delta=\frac{f_{\max }}{K+1}
$$

3. Dado que la forma de cada filtro es triangular, la escala lineal de frecuencias establece los puntos de inicio, intermedio y final de cada filtro. Dicha escala queda dada por

$$
F_{\text {lineal }}(i)=(i-1) \Delta, \quad i=1, \ldots, K+2 .
$$

4. Se aplica la ecuación 2.1 a la escala de frecuencias lineal para obtener la correspondiente en escala Mel.

$$
F_{M E L}(i)=1000 \frac{\log \left(1+\frac{F_{\text {lineal }}(i)}{1000}\right)}{\log (2)}, \quad i=1, \ldots, K+2 .
$$

Esta escala contiene también los puntos de inicio, intermedio y final de cada filtro triangular. 
5. Cada filtro triangular tiene un traslape con el siguiente de manera que el punto intermedio de uno es el inicio del siguiente. El filtro es aplicado al espectro obtenido de cada segmento de voz del registro $S_{j}(n)_{v o z}$; cada filtro se multiplica por los valores del espectro correspondientes y los resultados son promediados, de forma que se obtiene un vector espectral de longitud $K$ como salida del procesamiento.

La figura 2.10 muestra una vista final del banco de filtros en la escala Mel. Hay que resaltar que la aplicación de este filtro se realiza sobre un espectro FFT de 256 puntos de forma que es necesario corresponder los valores de dicha escala con el espectro discreto.

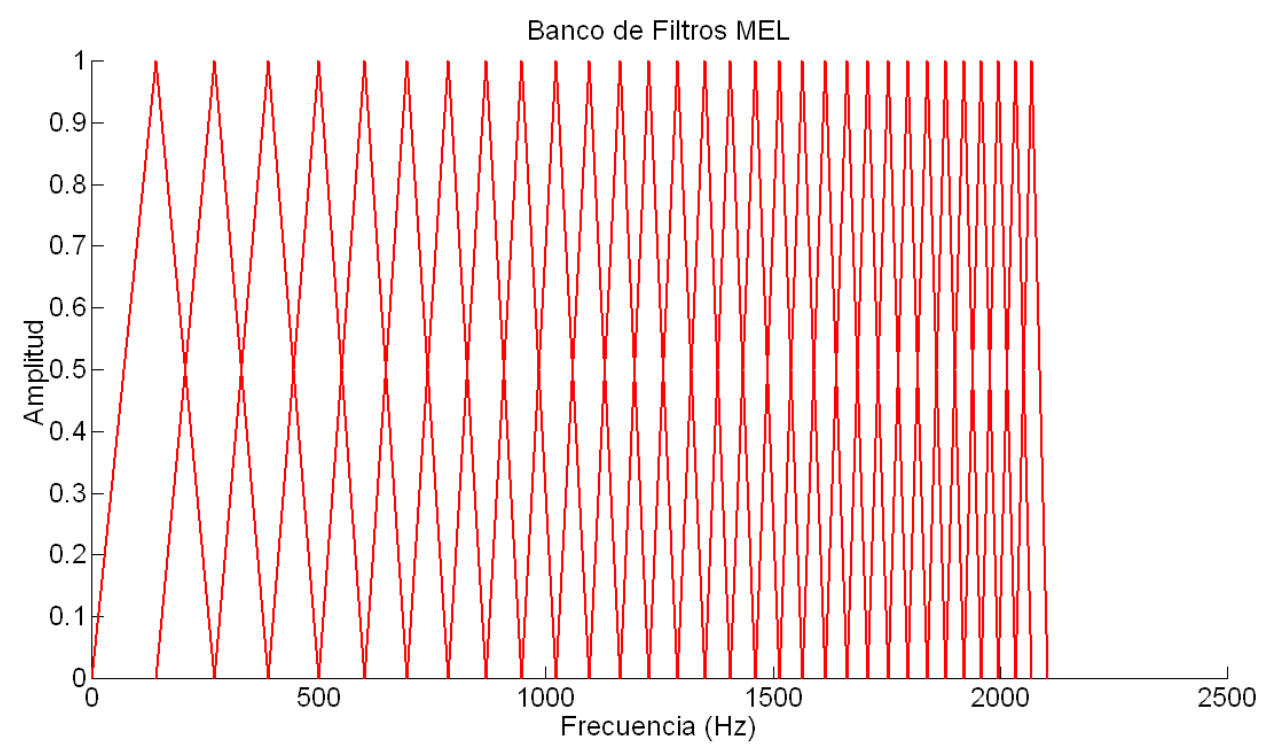

Figura 2.10: Gráfica correspondiente a un banco de 31 filtros en escala Mel.

\subsection{Máquinas de Soporte Vectorial}

Clasificar involucra necesariamente el concepto de conjunto; de manera general el proceso de clasificación consiste en realizar una separación de los elementos de un conjunto $C$ en diferentes subconjuntos $C_{i}, i=1, \ldots, P$, denominados clases, con base en la medición de las características que los elementos de $C$ poseen. Una vez que se determinan las propiedades de los subconjuntos en los que se clasificará al conjunto original (modelos), los elementos de éste son comparados con cada uno de los modelos, para establecer a cual de ellos pertenecen. Matemáticamente este proceso puede entenderse como una función que mapea el conjunto $C$ al conjunto de clases $\left\{C_{i}\right\}_{i=1}^{P}$. Se parte de la hipótesis de que, sin importar la naturaleza del conjunto $C$, sus elementos pueden ser representados de forma numérica. 
Esta representación puede ser en $\mathbb{R}^{n}$, para algún $n \in \mathbb{N}$. Sin embargo bajo este planteamiento, la labor de clasificación no guarda dificultad alguna, ésta surge, por ejemplo, cuando se considera que los elementos del conjunto $C$ son resultado de un conjunto finito de variables aleatorias en $\mathbb{R}^{n}$ denotado por

$$
C=\left\{X_{1}, X_{2}, \ldots, X_{k}\right\},
$$

donde $X_{j}$ es una variable aleatoria discreta infinita o continua. Si consideramos que las clases $C_{i}$ son una partición de $C$ entonces, dadas las hipótesis, el proceso de clasificación puede no ser exhaustivo, por lo que, de manera práctica, $C$ es formado con subconjuntos de valores representativos de cada una de las variables aleatorias $X_{j}$. Así, el objetivo de una Máquina de Soporte Vectorial (Support Vector Machine, SVM) consiste en modelar en cierta forma el comportamiento de cada una de las variables aleatorias $X_{j}$, de forma que se pueda determinar, dado un vector propuesto, a cual de ellas pertenece.

En particular, para la clasificación de voz puede considerarse, sin pérdida de generalidad, que el conjunto $C$ está formado por dos variables aleatorias, es decir que

$$
C=\left\{X_{1}, X_{2}\right\}
$$

Es posible representar a cada elemento del conjunto $C$, de la siguiente forma:

$$
\left(x_{j}, y_{j}\right), j=1, \ldots, l,
$$

donde $x_{j} \in \mathbb{R}^{n}, y_{j} \in\{-1,1\}$ y $l$ es la cardinalidad de $C$. Si suponemos que tomamos una muestra representativa de cada una de las variables aleatorias, la representación dada previamente permite establecer al conjunto $C$ de la siguiente forma:

$$
\begin{array}{r}
C=C_{1} \cup C_{2}, \quad C_{1} \cap C_{2}=\emptyset, \\
C_{1}=\left\{x_{1}, \ldots, x_{k}\right\}, \\
C_{2}=\left\{x_{k+1}, \ldots, x_{l}\right\} .
\end{array}
$$

Por las consideraciones hechas, la distribución de los puntos que conforman a $C_{1}$ y $C_{2}$ es desconocida a priori. Podemos entonces considerar, de forma general, dos casos; cuando $C_{1}$ y $C_{2}$ son linealmente separables y cuando no lo son [50]. La teoría dada a continuación puede ampliarse consultando [45] y [50].

\subsubsection{Conjuntos Separables Linealmente}

Se dice que $C_{1}$ y $C_{2}$ son linealmente separables cuando existe un hiperplano en $\mathbb{R}^{n}$ determinado por un vector $w$ perpendicular al mismo de forma que

$$
w \cdot x+b=0, \quad b \in \mathbb{R}
$$


para cualquier punto $x$ en el hiperplano y además

$$
\begin{aligned}
& w \cdot x_{i}+b>0, \quad \forall x_{i} \in C_{1}, \\
& w \cdot x_{j}+b<0, \quad \forall x_{j} \in C_{2} .
\end{aligned}
$$

Puede obtenerse fácilmente que si $C_{1}$ y $C_{2}$ son linealmente separables entonces la existencia de un hiperplano tal, determinado por un vector $w$, no es única, de hecho existen una infinidad de tales vectores. Así, es necesario establecer un criterio que permita determinar cual de ellos se tomará para la clasificación. También es inmediato que si ambos conjuntos son separables entonces existe una distancia mínima entre ambos conjuntos. La figura 2.11 presenta un esquema del caso que se expone.

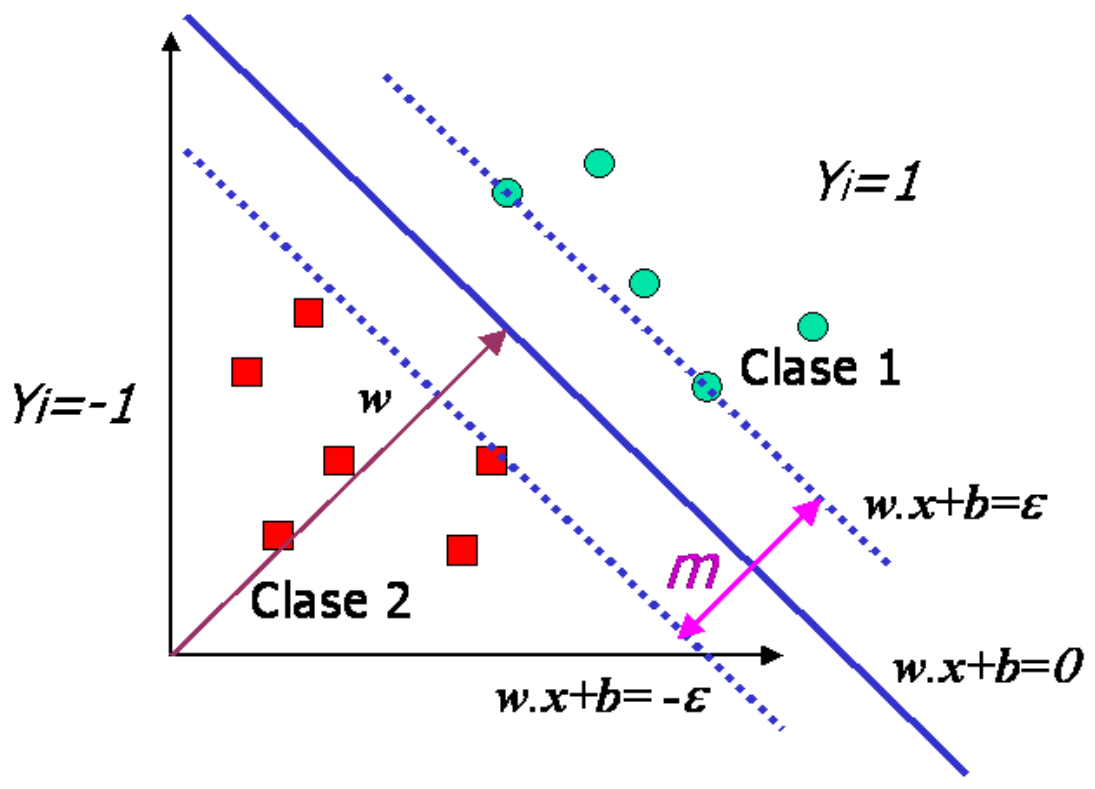

Figura 2.11: Esquema de dos conjuntos de vectores linealmente separados mediante un hiperplano que maximiza el margen $m$.

De la misma, podemos observar la construcción de dos hiperplanos paralelos al original, determinado éste por $w$, los cuales delimitan un margen entre los conjuntos y cuya magnitud $m$ podemos relacionar con dichos hiperplanos de la siguiente forma: Supongamos que $w$ está contenido en el hiperplano inferior entonces sucede que

$$
w \cdot w+b=-\varepsilon,
$$

para alguna constante $\varepsilon>0$. Entonces el vector dado por

$$
w+\frac{w}{\|w\|} m,
$$


está incluido en el hiperplano superior, lo cual implica que

$$
w \cdot\left(w+\frac{w}{\|w\|} m\right)+b=\varepsilon .
$$

Restando las ecuaciones 2.15 y 2.17 y simplificando obtenemos

$$
\|w\| m=2 \varepsilon \Rightarrow m=\frac{2 \varepsilon}{\|w\|} .
$$

Dada la igualdad 2.18 se infiere que si deseamos maximizar la magnitud del margen $m$ es necesario minimizar la magnitud de $w$. Retomando nuevamente la notación $\left(x_{j}, y_{j}\right)$ con $y_{j} \in\{1,-1\}$, para cada uno de los vectores $x_{j}$ de $C$, entonces el problema de encontrar un hiperplano que separe a $C_{1}$ y a $C_{2}$, maximizando el margen de separación entre dichos conjuntos, queda planteado como:

$$
\begin{gathered}
\min \left\{f(w)=\frac{1}{2 \varepsilon}\|w\|^{2}\right\} \\
y_{i}\left(w \cdot x_{i}+b\right) \geq \varepsilon, \\
i=1, \ldots, l, \\
\varepsilon>0, b \in \mathbb{R} .
\end{gathered}
$$

Este es un problema de optimización de tipo cuadrático sujeto a $l$ restricciones en $\mathbb{R}^{n}$ y cuya solución puede darse a partir del uso de la teoría de multiplicadores de Lagrange en $n$ variables.

Denotemos las restricciones que aparecen en el problema 2.19 de la siguiente forma:

$$
g_{i}(w)=y_{i}\left(w \cdot x_{i}+b\right), \quad i=1, \ldots, l,
$$

entonces si denotamos por $w_{k}$ a la k-ésima entrada de $w$ sabemos que se cumple

$$
\begin{array}{rr}
\frac{\partial f}{\partial w_{k}}(w)=\sum_{i=1}^{l} \alpha_{i} \frac{\partial g_{i}}{\partial w_{k}}(w), \quad k=1, \ldots, l \\
\alpha_{i} g_{i}(w)=\varepsilon, \quad i=1, \ldots, l,
\end{array}
$$

para ciertas constantes $\alpha_{i} \in \mathbb{R}$ a determinar. Sustituyendo en la ecuación 2.21 a la función $f$ y a las funciones $g_{i}$ se tiene el siguiente desarrollo:

$$
\begin{array}{r}
\frac{\partial f}{\partial w_{k}}=\frac{1}{\varepsilon} w_{k}=\sum_{i=1}^{l} \alpha_{i} \frac{\partial g_{i}}{\partial w_{k}}=\sum_{i=1}^{l} \alpha_{i} y_{i} x_{i}(k) \Rightarrow \\
w=\varepsilon \sum_{i=1}^{l} \alpha_{i} y_{i} x_{i} .
\end{array}
$$


Por otro lado de la igualdad 2.22 se tiene que

$$
\frac{\partial \sum_{i=1}^{l} \alpha_{i} g_{i}(w)}{\partial b}=0 \Rightarrow \sum_{i=1}^{l} \alpha_{i} y_{i}=0
$$

Por lo tanto la solución al sistema 2.19 está dada por

$$
\begin{array}{r}
w=\varepsilon \sum_{i=1}^{l} \alpha_{i} y_{i} x_{i} \\
\sum_{i=1}^{l} \alpha_{i} y_{i}=0
\end{array}
$$

Para obtener el valor de cada una de las constantes $\alpha_{i}$ sustituimos la ecuación 2.23 en las restricciones iniciales 2.22 con lo que se obtiene la siguiente ecuación:

$$
\alpha_{j} y_{j}\left(\varepsilon x_{j} \cdot \sum_{i=1}^{l} \alpha_{i} y_{i} x_{i}+b\right)=\varepsilon, \quad j=1, \ldots, l .
$$

Derivando parcialmente cada una de las ecuaciones dadas en 2.25 con respecto a $\alpha_{j}$, se obtiene que

$$
\alpha_{j}\left\|x_{j}\right\|^{2}+y_{j} \sum_{i=1}^{l} \alpha_{i} y_{i} x_{i} \cdot x_{j}=-\frac{y_{j} b}{\varepsilon}, \quad j=1, \ldots, l .
$$

Las igualdades descritas en 2.26 conforman un sistema lineal de $l$ ecuaciones con incógnitas $\alpha_{i}, i=1, \ldots, l$, por lo que se tiene un sistema de la forma $A \alpha=B$, con $A \in \mathbb{R}^{l x l}$ y $B \in \mathbb{R}^{l}$, siendo $\alpha$ el vector de incógnitas. Este sistema tiene solución única sólo si $A$ tiene inversa. Es importante recordar que el valor $l$ es la cardinalidad del conjunto $C$, por lo que la complejidad en la obtención de una solución mediante alguna implementación, dependerá del orden de dicho conjunto. Los valores de las constantes $\alpha_{i}$ quedarán en términos de $b$ y $\varepsilon$, de los cuales uno de ellos puede ser propuesto y el otro determinado, por ejemplo, con la ecuación 2.15.

Así, el vector $w$ dado en la ecuación 2.23 es conocido como el vector de soporte del hiperplano que separa a $C_{1}$ y a $C_{2}$, de donde deriva el nombre de Máquinas de Soporte Vectorial.

\subsubsection{Conjuntos No Separables Linealmente}

Puede considerarse que el sistema lineal de ecuaciones dado en 2.26, es una conclusión al tratar el caso de dos conjuntos $C_{1}$ y $C_{2}$, separables linealmente. Sin embargo dicho sistema 
puede ser obtenido también para cualesquiera dos conjuntos en $\mathbb{R}^{n}$. Así, podemos decir que $C_{1}$ y $C_{2}$ no son separables linealmente cuando la matriz correspondiente al sistema 2.26 no tenga solución, en cuyo caso no es posible la construcción de un hiperplano que satisfaga las condiciones del problema planteado en 2.19.

El tratamiento para el caso de dos conjuntos no separables linealmente consiste en utilizar una función $\phi: \mathbb{R}^{n} \rightarrow \mathbb{R}^{m}$, con $n, m \in \mathbb{N}, m \geq n$ ó $m=\infty$. Dicha función mapea a los conjuntos $C_{1}=\left\{x_{1}, \ldots, x_{k}\right\}$ y $C_{2}=\left\{x_{k+1}, \ldots, x_{l}\right\}$, a un espacio de mayor dimensión, donde podemos denotarlos por $\Gamma_{1}=\left\{\phi\left(x_{1}\right), \ldots, \phi\left(x_{k}\right)\right\}$ y $\Gamma_{2}=\left\{\phi\left(x_{k+1}\right), \ldots, \phi\left(x_{l}\right)\right\}$ respectivamente. El objetivo de realizar este mapeo es que los conjuntos obtenidos $\Gamma_{1}$ y $\Gamma_{2}$ sean separables linealmente o en su caso se minimice el error mediante la separación con un hiperplano, es decir que el número de vectores clasificados incorrectamente sea mínimo. La figura 2.12 esquematiza la operación ideal de la función $\phi$ sobre un conjunto dado.

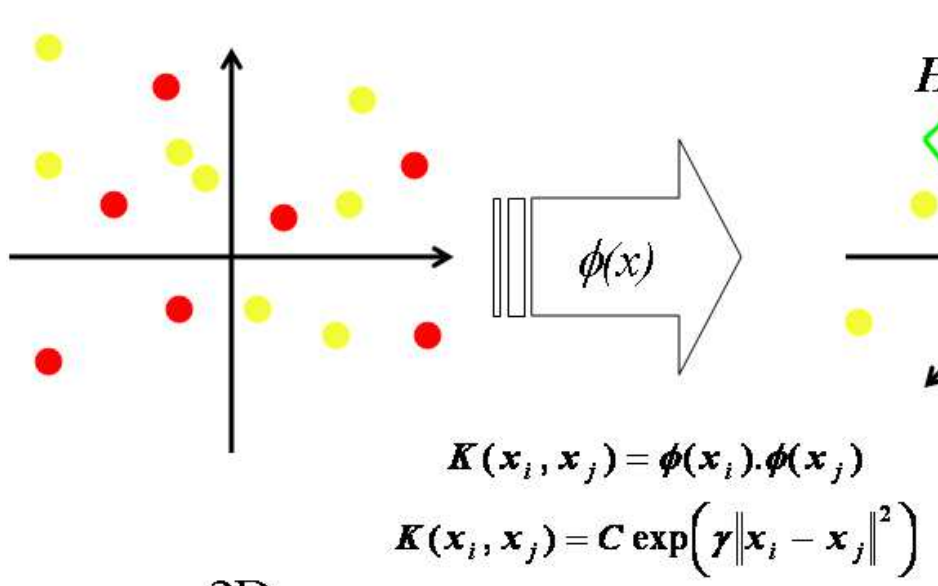

$2 \mathrm{D}$

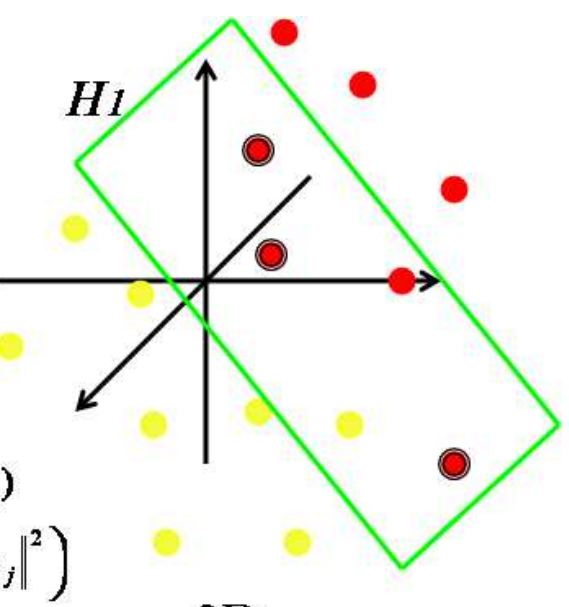

$3 \mathrm{D}$

Figura 2.12: Esquema de la transformación de dos conjuntos no separables linealmente mediante la función $\phi$.

Como se mencionó anteriormente, idealmente la función $\phi$ mapea los conjuntos de vectores no separables linealmente a un espacio de mayor dimensión. Éstos vectores son separables linealmente y la solución al problema original mediante el procedimiento explicado en la sección 2.4.1, es un hiperplano $H_{1} \subset \mathbb{R}^{m}$. En este caso se considera que existen vectores de los conjuntos $\Gamma_{1}$ y $\Gamma_{2}$ que se encuentran contenidos en $H_{1}$. A dichos vectores se les conoce como vectores de soporte. Para obtener una solución en el espacio $\mathbb{R}^{n}$ original, se realiza el mapeo inverso de los vectores de soporte, los cuales determinarán las fronteras que separarán a los conjuntos $C_{1}$ y $C_{2}$. Se considera que los vectores que determinan estas fronteras conforman el modelo para $C_{1}$ (o equivalentemente para $C_{2}$ ). 
No es posible determinar a priori, dados dos conjuntos de vectores $C_{1}, C_{2} \subset \mathbb{R}^{n}$, una función $\phi$ que cumpla los objetivos descritos previamente, por lo que el procedimiento para determinarla no es constructivo. Por tanto el tratamiento para este caso está basado en la realización de ensayos con funciones $\phi$ conocidas. Así, la función $\phi$ es de especial importancia en la solución del problema de clasificación.

Del procedimiento dado en 2.4.1, puede observarse que las operaciones con vectores involucran el producto punto o producto interno canónico en $\mathbb{R}^{n}$. Éste proporciona una función que determina una norma y a su vez una métrica para el espacio:

$$
\begin{array}{r}
\|x\|=\sqrt{x \cdot x} \\
d(x, y)=\|x-y\| .
\end{array}
$$

Tales norma y métrica, respectivamente, son empleadas también al separar los conjuntos $\Gamma_{1}$ y $\Gamma_{2}$ en $\mathbb{R}^{m}$. Así que el problema análogo al 2.19, planteado en este nuevo espacio es

$$
\begin{gathered}
\min \left\{f(w)=\frac{1}{2 \varepsilon}\|w\|^{2}, \quad w \in \mathbb{R}^{m}\right\} \\
y_{i}\left(w \cdot \phi\left(x_{i}\right)+b\right) \geq \varepsilon \\
i=1, \ldots, l \\
\varepsilon>0, b \in \mathbb{R} .
\end{gathered}
$$

Por lo anterior la ecuación análoga a 2.26 en este nuevo espacio está dada por

$$
\alpha_{j}\left\|\phi\left(x_{j}\right)\right\|^{2}+y_{j} \sum_{i=1}^{l} \alpha_{i} y_{i} \phi\left(x_{i}\right) \cdot \phi\left(x_{j}\right)=-\frac{y_{j} b}{\varepsilon}, \quad j=1, \ldots, l .
$$

Se infiere de la ecuación 2.30, que la función $\phi$ puede ser vista como una que modifica la norma y métrica del espacio original (dadas en 2.27 y 2.28), por las siguientes:

$$
\begin{gathered}
\|x\|_{\phi}=\sqrt{\phi(x) \cdot \phi(x)}, \\
d_{\phi}(x, y)=\|x-y\|_{\phi} .
\end{gathered}
$$

A la función definida por

$$
K\left(x_{i}, x_{j}\right)=\phi\left(x_{i}\right) \cdot \phi\left(x_{j}\right),
$$

se le conoce como función núcleo y su uso es más importante que el de la propia función $\phi$, de la cual no se requiere su conocimiento en forma explícita, ya que es suficiente, como lo 
muestra la ecuación 2.30, con establecer la función núcleo $K$ para obtener una solución.

Algunos ejemplos de funciones núcleo que han sido sugeridas o empleadas en problemas de clasificación son las siguientes [22]:

\section{Lineal:}

$$
K\left(x_{i}, x_{j}\right)=x_{i} \cdot x_{j}
$$

Polinomial:

$$
K\left(x_{i}, x_{j}\right)=\left(\gamma x_{i} \cdot x_{j}+r\right)^{d}, \quad \gamma>0, r, d \in \mathbb{R} .
$$

Función de Base Radial: (Radial Basis Function, RBF)

$$
K\left(x_{i}, x_{j}\right)=c \exp \left(-\gamma\left\|x_{i}-x_{j}\right\|^{2}\right), \quad \gamma>0, c \in \mathbb{R} .
$$

Sigmoide:

$$
K\left(x_{i}, x_{j}\right)=\tanh \left(\gamma x_{i} \cdot x_{j}+r\right), \gamma, r \in \mathbb{R} .
$$

Aún cuando existen diferentes funciones núcleo, es común el uso de la Función de Base Radial, por los resultados obtenidos durante la clasificación [9], [22]. Sin embargo puede optarse por el uso de otros núcleos dependiendo de los resultados obtenidos para un caso particular.

Es importante mencionar que dado el origen y la naturaleza de los vectores obtenidos en el proceso de extracción, se espera tratar con un caso de conjuntos no separables linealmente, por lo que en este trabajo se propone utilizar inicialmente la función de base radial (dada en 2.36), para realizar la clasificación. 


\section{Capítulo 3}

\section{Desempeño del Sistema}

En el proceso de verificación de un hablante, es necesaria la conformación de un modelo que corresponda a la voz. Como se mostró en el capítulo 2, el sistema de verificación basado en Máquinas de Soporte Vectorial, requiere la extracción de vectores de características, mismos que son obtenidos mediante el análisis cepstral de los registros de voz. El conjunto total de vectores de cada usuario, puede ser dividido en subconjuntos para la creación de modelos de la voz de los hablantes y también para la realización de pruebas que evalúen al sistema. El subconjunto de vectores utilizado para conformar el modelo de un usuario se denomina conjunto de entrenamiento, éste puede considerarse como la clase o conjunto $E_{i}$. Para el proceso de clasificación, la otra clase se forma a partir de vectores del resto de los usuarios, válidos e inválidos. Este conjunto se conoce como Background del usuario, que será denotado por $B_{i}$. El sistema de clasificación determina entonces los vectores de soporte que separan a ambas clases, minimizando los errores de clasificación.

Una vez creado el modelo del usuario, pueden realizarse pruebas que lo validen, por ejemplo clasificando un conjunto $P_{i} \neq E_{i}$, de vectores de características del usuario. El objetivo es determinar el porcentaje de aciertos con un conjunto distinto al de entrenamiento. La cardinalidad del conjunto de prueba puede variarse para observar la efectividad en la clasificación. $P_{i}$ puede consistir también de vectores de características de usuarios no válidos, lo cual prueba el sistema ante este tipo de solicitudes.

En este capítulo se describen las técnicas empleadas para la formación de conjuntos de entrenamiento y conjuntos de prueba así como los resultados obtenidos de las pruebas. Adicionalmente se presenta un procedimiento para evaluar el desempeño del sistema basado en curvas DET. Se presenta por último la validación de los modelos creados, mediante la realización de pruebas con muestras de voz de los usuarios en tiempo real. 


\subsection{Entrenamiento y Pruebas}

Existen diferentes aspectos que deben considerarse para la formación de conjuntos de entrenamiento y prueba:

Cardinalidad de los conjuntos $E_{i} \mathbf{y} B_{i}$. Inicialmente no se tiene un parámetro de referencia para asignar la cantidad de vectores adecuado para formar tales conjuntos o, incluso, si dado el sistema de clasificación usado, existen valores óptimos. Uno de los objetivos de este trabajo consiste en observar el comportamiento del sistema, en relación con los valores de cardinalidad propuestos para estos conjuntos.

Cardinalidad de $P_{i}$. El conjunto de prueba más simple consta de un sólo vector, el cual puede corresponder a un usuario válido o a uno inválido. Al incrementar la cardinalidad del conjunto de prueba se puede observar el comportamiento del sistema de verificación y así establecer un valor óptimo: el número mínimo de vectores para la operación aceptable del sistema.

Selección de los vectores de $E_{i}$. Como se ha planteado $E_{i} \subset\left\{x_{i k}\right\}_{k=1}^{l}$, sin embargo existen diferentes formas de realizar una selección de los vectores que forman al conjunto $E_{i}$. Por ejemplo se puede realizar una selección pseudoaleatoria. También pueden considerarse vectores que correspondan a una misma secuencia de voz del registro $S_{i}$. Otra técnica conocida es la de validación cruzada (Cross Validation), en la cual se realiza una partición del conjunto total de vectores, en $K$ subconjuntos de igual cardinalidad. Posteriormente se toma cada uno de los $K$ subconjuntos como conjunto de entrenamiento y se obtiene el porcentaje de acierto con los $K-1$ conjuntos restantes. El conjunto de entrenamiento que menor porcentaje de error proporcione, será considerado para conformar el modelo final del usuario.

Selección de los vectores de $B_{i}$. Dada la definición de $B_{i}$ y su uso en la clasificación, la formación de este conjunto requiere que los vectores incluidos sean representativos del resto de los usuarios a $U_{i}$. Por ello la selección de éstos puede ser a partir de la misma técnica empleada para formar a cada uno de los $E_{i}$. Sin embargo debe realizarse un proceso de selección tal que minimice la cardinalidad de $B_{i}$, pues ésta incrementa también la complejidad del problema de clasificación, como se vió en la sección 2.4.2.

A continuación se da una descripción de las características bajo las que se realizó la formación de modelos y las pruebas al sistema de verificación basado en la base Voces-MCyTI.

Dado un usuario $U_{i}$, el conjunto total de sus vectores de características se denota por $\left\{x_{i k}\right\}_{k=1}^{l}$. Éstos son obtenidos mediante el procesamiento de la señal $S_{i}$ descrito en la sección 2.3. De esta forma se obtienen 17 conjuntos de vectores de características de la misma cardinalidad. Los conjuntos de entrenamiento $E_{i}$, del Background $B_{i}$ y de prueba $P_{i}$, satisfacen 


$$
\begin{array}{r}
E_{i} \cup P_{i}=\left\{x_{i k}\right\}_{k=1}^{l}, E_{i} \cap P_{i}=\emptyset \\
B_{i} \subset\left\{x_{1 k}\right\}_{k=1}^{l} \cup, \ldots, \cup\left\{x_{(i-1) k}\right\}_{k=1}^{l} \cup\left\{x_{(i+1) k}\right\}_{k=1}^{l} \cup, \ldots, \cup\left\{x_{17 k}\right\}_{k=1}^{l} .
\end{array}
$$

De esta forma el conjunto $\left\{x_{i k}\right\}_{k=1}^{l}$ es dividido para obtener el modelo $M_{i}$ del usuario, proceso que se conoce como entrenamiento. El resto de los vectores es empleado para realizar pruebas que validen el modelo creado.

La base Voces-MCyTI consta de 17 registros, de ellos $S_{1}, \ldots, S_{15}$ serán considerados como usuarios válidos y los dos restantes como usuarios no incluidos en el sistema. El tiempo de los registros $S_{i}(n)_{v o z}$, luego de la supresión de silencios, se ubicó en un intervalo de 33 a 39 segundos. Con el fin de uniformizar los registros de voz, se consideró procesar sólo los primeros 32.15 segundos de cada uno de ellos. De esta forma se obtuvieron un total de 1606 vectores para cada hablante, es decir $l=1606$. La forma de selección y formación de los conjuntos $E_{i}, P_{i}$ y $B_{i}$ estuvo basada en los siguientes puntos:

1. Inicialmente se consideró la formación secuencial de los conjuntos $E_{i}$ y $P_{i}$, es decir se formaron conjuntos de vectores asociados a tramas de voz, en el mismo orden en el que aparecen en los registros y con base en la ecuación 3.1. Sin embargo los resultados de clasificación obtenidos mostraron que el sistema se volvía dependiente del texto, al presentar un bajo porcentaje de clasificación para las frases del conjunto $P_{i}$.

2. Para evitar la dependencia de los modelos de los hablantes a las frases, se realizó un reordenamiento aleatorio de los vectores, respecto del obtenido originalmente, posterior al procesamiento.

3. La formación de los conjuntos $E_{i}$ se hizo considerando un $78 \%$ del total de los vectores. Con este porcentaje se obtiene un número de vectores aproximado al de otros problemas de clasificación resueltos mediante Máquinas de Soporte Vectorial [22]. Debido al reordenamiento aleatorio de los vectores, se consideró que el proceso de selección carecía de relevancia, por lo que se seleccionaron los primeros 1,252 vectores.

4. A fin de evaluar el comportamiento de los modelos generados respecto al número de vectores de prueba, se consideró la formación de dos conjuntos de $P_{i}^{1}$ y $P_{i}^{2}$, con 252 y 102 vectores, respectivamente.

5. La tabla 3.1 sintetiza las características de los conjuntos de entrenamiento y prueba.

El número de vectores de $P_{i}^{2}$ se asignó con base en el tiempo estimado requerido por el sistema para verificar a un hablante. De esta manera dicho valor corresponde a la señal $S_{i}(n)_{v o z}$, sin intervalos de silencio. De forma real, el tiempo aproximado de grabación sería de 4 s. 


\begin{tabular}{|c|c|c|}
\hline Conjunto & No. de vectores & Tiempo de $S_{j}(n)_{v o z}$ \\
\hline$E_{i}$ & 1,252 & $25.05 \mathrm{~s}$ \\
\hline$P_{i}^{1}$ & 252 & $5.05 \mathrm{~s}$ \\
\hline$P_{i}^{2}$ & 102 & $2.05 \mathrm{~s}$ \\
\hline
\end{tabular}

Tabla 3.1: Características de los conjuntos de entrenamiento y prueba.

6. Para la formación de los conjuntos $B_{i}$, se consideró que la cardinalidad de éste debía ser similar a la de los conjuntos $E_{i}$, pues al incrementar su valor se observó un incremento en el tiempo requerido por el sistema de clasificación. Por lo anterior y dado un usuario $U_{i}$ válido, se seleccionaron los primeros 200 vectores de cada conjunto $\left\{x_{j k}\right\}_{k=1}^{l}, j=$ $1, \ldots,(i-1),(i+1), \ldots, 15$, sin considerar los registros de los usuarios no incluidos, es decir $S_{16}$ y $S_{17}$. La selección se hizo sobre los conjuntos una vez aplicado el reorden aleatorio. De esta forma cada conjunto $B_{i}$ tiene 2800 vectores de características. El objetivo de excluir los registros $S_{16}$ y $S_{17}$ en la formación de los $B_{i}$, es para observar, posteriormente, el desempeño del sistema ante peticiones de usuarios no incluidos en forma alguna.

\subsubsection{Entrenamiento del Sistema}

El entrenamiento del sistema de clasificación se realiza a partir de las parejas $\left(E_{i}, B_{i}\right)$, lo cual genera un modelo $M_{i}$ correspondiente al usuario. Para la generación de estos modelos se utilizó el software Libsvm [22] basado en Máquinas de Soporte Vectorial. Este software ha sido utilizado para mejorar porcentajes de clasificación en diferentes problemas y cuenta con herramientas que permiten un manejo adecuado de los datos. El procedimiento seguido para el entrenamiento del sistema de verificación se describe a continuación:

1. Se realiza la normalización de los conjuntos de vectores $E_{i}$ y $B_{i}$ con base en la siguiente ecuación:

$$
N_{i}(x(n))=\frac{1}{x_{\max }^{i}-x_{\min }^{i}}\left(2 x(n)-\left(x_{\max }^{i}+x_{\min }^{i}\right)\right),
$$

donde $x_{\text {max }}^{i}$ y $x_{\text {min }}^{i}$ son el máximo y mínimo del conjunto dado por $E_{i} \cup B_{i}$, considerando cada entrada de los vectores, y $x(n)$ es la n-ésima entrada del vector de características $x$. La ecuación 3.3 mapea cada conjunto de vectores $\left\{x_{i k}\right\}_{k=1}^{l}$ dentro del intervalo $[-1,1]$. El registro de esta normalización se almacena en un archivo que se denomina rango_i.

2. Se procede al entrenamiento del sistema utilizando los nuevos conjuntos $E_{i}$, $B_{i}$ normalizados. Las características del modelo $M_{i}$ correspondiente se almacenan en un archivo con extensión .model. Éste contiene los datos respecto de las fronteras (vectores de soporte) determinadas por el sistema mediante Máquinas de Soporte Vectorial. 
3. El núcleo utilizado para la clasificación es la función de base radial (Ecuación 2.36), cuyos parámetros $c$ y $\gamma$ pueden ser ajustados para mejorar los porcentajes de acierto en la clasificación. El software Libsvm cuenta con una herramienta para mejorar el porcentaje de acierto mediante la búsqueda de valores óptimos para $c$ y $\gamma$ en una matriz o rejilla de valores, dado un conjunto de vectores de prueba. En las pruebas realizadas con esta herramienta de búsqueda, se observó mejorías poco significativas en los porcentajes de acierto, a coste de un incremento en el tiempo de clasificación del orden de horas. En la sección siguiente se presentan los detalles de estas pruebas. Con base en esto, el entrenamiento se realizó con $c=32$ y $\gamma=1$, los cuales mostraron un mejor porcentaje de clasificación, para todos los usuarios.

La figura 3.1 esquematiza el procedimiento de generación de modelos para cada uno de los usuarios.

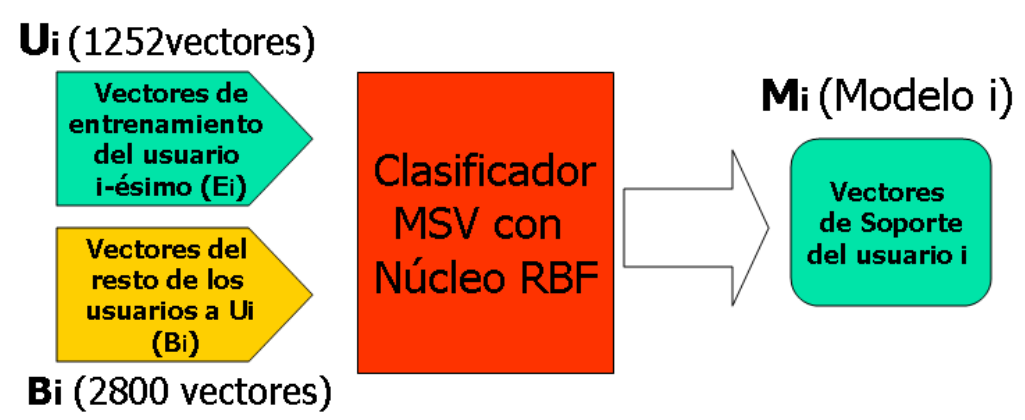

Figura 3.1: Procedimiento de generación de modelos $M_{i}$.

Una vez formados los 15 modelos $M_{i}$ se procedió a realizar las pruebas descritas en la siguiente sección.

\subsubsection{Pruebas y Resultados}

Las primeras pruebas realizadas tuvieron el objetivo de determinar valores óptimos para los parámetros c y $\gamma$ de la función de base radial, utilizada como núcleo en la clasificación. En estas pruebas se determina, dada $\left(E_{i}, B_{i}\right)$, los valores de $c_{i}$ y $\gamma_{i}$ que maximizan el porcentaje de aciertos en la clasificación del conjunto $P_{i}^{1}$, al realizar una búsqueda de éstos en una matriz o rejilla de valores $\left(c_{j}, \gamma_{k}\right)$. La búsqueda se inicia a partir de un intervalo dado para cada parámetro, por lo que el resultado obtenido no es necesariamente un óptimo global. Estos valores son utilizados posteriormente para obtener el porcentaje de clasificación con $P_{i}^{1}$ y $P_{i}^{2}$, mismos que fueron normalizados con la ecuación 3.3. Es importante recordar que una vez conformado el modelo de un usuario, el proceso de prueba para obtener un porcentaje de acierto consiste en ingresar al sistema un vector $x_{i k} \in P_{i}$, el cual es asignado al usuario 
$U_{i}$, lo que es considerado como un acierto; o a su complemento $\left\{U_{i}\right\}^{c}$, lo que se considera un error. De esta forma, el porcentaje de acierto se calcula como el cociente del número de vectores clasificados correctamente entre la cardinalidad del conjunto de prueba.

$$
\operatorname{Acierto}(\%)=100 \times \frac{\text { No_Aciertos }}{\text { No_Total_Vectores }} .
$$

La tabla 3.2 presenta los resultados de las pruebas descritas previamente.

\begin{tabular}{|c|c|c|c|c|c|}
\hline Usuario & Modelo & $c_{i}$ & $\gamma_{i}$ & Aciertos $P_{i}^{1}(\mathbf{\%})$ & Aciertos $P_{i}^{2}(\boldsymbol{\%})$ \\
\hline$U_{1}$ & $M_{1}$ & 32 & 1.1 & 72.222 & 72.549 \\
\hline$U_{2}$ & $M_{2}$ & 64 & 0.8 & 83.333 & 69.607 \\
\hline$U_{3}$ & $M_{3}$ & 64 & 0.5 & 71.825 & 67.647 \\
\hline$U_{4}$ & $M_{4}$ & 32 & 0.6 & 71.031 & 61.764 \\
\hline$U_{5}$ & $M_{5}$ & 64 & 1.1 & 61.111 & 64.705 \\
\hline$U_{6}$ & $M_{6}$ & 96 & 1.6 & 57.936 & 60.784 \\
\hline$U_{7}$ & $M_{7}$ & 96 & 2.0 & 58.333 & 56.862 \\
\hline$U_{8}$ & $M_{8}$ & 32 & 2.1 & 65.873 & 71.568 \\
\hline$U_{9}$ & $M_{9}$ & 16 & 1.3 & 68.254 & 68.627 \\
\hline$U_{10}$ & $M_{10}$ & 64 & 1.4 & 59.523 & 53.921 \\
\hline$U_{11}$ & $M_{11}$ & 96 & 0.9 & 65.079 & 63.725 \\
\hline$U_{12}$ & $M_{12}$ & 64 & 0.77 & 72.619 & 59.803 \\
\hline$U_{13}$ & $M_{13}$ & 64 & 1.97 & 59.920 & 52.941 \\
\hline$U_{14}$ & $M_{14}$ & 16 & 1.65 & 65.873 & 69.607 \\
\hline$U_{15}$ & $M_{15}$ & 16 & 1.15 & 76.190 & 82.352 \\
\hline
\end{tabular}

Tabla 3.2: Valores óptimos de $c$ y $\gamma$ para cada usuario.

De los resultados obtenidos puede observarse que hay una dependencia entre los valores $c_{i}, \gamma_{i}$ y el conjunto $P_{i}^{1}$, a partir del cual se obtienen. Esto se deduce a partir de los porcentajes de acierto obtenidos con el conjunto $P_{i}^{2}$ que son, en general, menores que los de $P_{i}^{1}$. Esta disminución en el porcentaje de aciertos así como el tiempo requerido para la búsqueda, permiten excluir este procedimiento como parte del sistema de verificación, pues no existe un beneficio significativo en la clasificación para conjuntos de prueba distintos a $P_{i}^{1}$.

Como parte principal de las pruebas realizadas al sistema de verificación, se sometió cada conjunto de prueba $P_{i}^{1}, i=1, \ldots, 15$, a ser clasificado utilizando cada uno de los modelos $M_{i}, i=1, \ldots, 15$. Esta prueba puede entenderse de la siguiente forma:

Clasificación de $P_{i}^{1}$ con $M_{i}$. Se clasifica el primer conjunto de vectores de prueba del usuario $U_{i}$ con su modelo correspondiente. En este caso el sistema trata la petición 
de un usuario válido respecto de $M_{i}$, por lo que el porcentaje de acierto obtenido mide el desempeño o eficiencia del modelo generado para verificar al usuario.

Clasificación de $P_{j}^{1}$ con $M_{i}, i \neq j$. Se clasifica el primer conjunto de prueba del usuario $U_{j}$, con el modelo de otro usuario $U_{i}$ distinto. En este caso el sistema trata con una petición de un usuario inválido respecto de $M_{i}$, por lo que el porcentaje obtenido mide el error cometido por el sistema ante este tipo de solicitudes.

Los modelos $M_{i}$ utilizados para estas pruebas, fueron generados con los valores asignados a los parámetros $c$ y $\gamma$. Los resultados obtenidos son reportados en las tablas 3.3 y 3.4. Los valores máximos obtenidos son resaltados en "negritas".

\begin{tabular}{|c|c|c|c|c|c|c|c|c|}
\hline$\cdot$ & $M_{1}$ & $M_{2}$ & $M_{3}$ & $M_{4}$ & $M_{5}$ & $M_{6}$ & $M_{7}$ & $M_{8}$ \\
\hline$P_{1}^{1}$ & $\mathbf{7 1 . 4 2 8}$ & 5.952 & 10.714 & 15.476 & 18.650 & 29.761 & 30.158 & 6.746 \\
\hline$P_{2}^{1}$ & 12.698 & $\mathbf{8 2 . 1 4 2}$ & 12.301 & 8.333 & 9.126 & 25.396 & 5.952 & 14.682 \\
\hline$P_{3}^{1}$ & 12.301 & 10.317 & $\mathbf{7 0 . 6 3 4}$ & 8.730 & 11.904 & 38.888 & 14.285 & 26.190 \\
\hline$P_{4}^{1}$ & 16.269 & 5.185 & 25.000 & $\mathbf{6 8 . 2 5 4}$ & 19.444 & 42.063 & 13.888 & 15.873 \\
\hline$P_{5}^{1}$ & 19.841 & 9.523 & 17.857 & 19.444 & $\mathbf{5 8 . 3 3 3}$ & 25.396 & 19.047 & 12.698 \\
\hline$P_{6}^{1}$ & 17.063 & 11.507 & 20.634 & 29.365 & 21.825 & $\mathbf{5 5 . 5 5 5}$ & 18.254 & 5.158 \\
\hline$P_{7}^{1}$ & 21.031 & 7.142 & 19.047 & 17.063 & 12.698 & 42.460 & $\mathbf{5 1 . 8 7 3}$ & 14.285 \\
\hline$P_{8}^{1}$ & 7.539 & 5.158 & 13.095 & 5.555 & 11.904 & 11.904 & 12.301 & $\mathbf{6 2 . 3 0 1}$ \\
\hline$P_{9}^{1}$ & 8.333 & 10.317 & 8.730 & 12.698 & 12.301 & 24.206 & 17.460 & 11.904 \\
\hline$P_{10}^{1}$ & 13.492 & 3.571 & 11.904 & 17.857 & 5.952 & 10.714 & 9.523 & 24.603 \\
\hline$P_{11}^{1}$ & 9.523 & 18.254 & 12.698 & 15.873 & 18.650 & 34.523 & 11.904 & 7.142 \\
\hline$P_{12}^{1}$ & 18.254 & 11.111 & 11.507 & 7.142 & 9.523 & 20.634 & 13.095 & 16.666 \\
\hline$P_{13}^{1}$ & 7.539 & 5.158 & 14.285 & 9.920 & 9.523 & 21.825 & 13.888 & 28.571 \\
\hline$P_{14}^{1}$ & 5.952 & 9.523 & 8.333 & 13.888 & 5.555 & 34.920 & 16.666 & 28.968 \\
\hline$P_{15}^{1}$ & 11.507 & 21.428 & 21.031 & 5.952 & 11.507 & 18.650 & 12.301 & 14.682 \\
\hline
\end{tabular}

Tabla 3.3: Porcentajes de clasificación del sistema de verificación de los conjuntos $P_{j}^{1}$ con los modelos $M_{i}$.

Como se observa, los mejores porcentajes de clasificación son obtenidos, invariablemente, cuando se verifica el conjunto de prueba del usuario con su modelo respectivo. A pesar de que en algunos casos el porcentaje de error puede ser significativo (vgr. $P_{10}^{1}, M_{9}$ ), los porcentajes de clasificación obtenidos permiten siempre determinar de forma clara, a que modelo y usuario corresponde cada conjunto de prueba.

Para observar la estabilidad del sistema se realizaron pruebas con los conjuntos $P_{i}^{2}$, cuya cardinalidad es de 102 vectores $\left(50 \%\right.$ menos que la de los conjuntos $\left.P_{i}^{1}\right)$. 


\begin{tabular}{|c|c|c|c|c|c|c|c|}
\hline$\cdot$ & $M_{9}$ & $M_{10}$ & $M_{11}$ & $M_{12}$ & $M_{13}$ & $M_{14}$ & $M_{15}$ \\
\hline$P_{1}^{1}$ & 13.889 & 10.317 & 21.428 & 35.714 & 13.492 & 3.968 & 23.809 \\
\hline$P_{2}^{1}$ & 12.301 & 9.126 & 21.031 & 19.841 & 5.158 & 5.158 & 19.444 \\
\hline$P_{3}^{1}$ & 6.746 & 13.492 & 15.476 & 12.698 & 11.904 & 9.126 & 17.857 \\
\hline$P_{4}^{1}$ & 25.793 & 15.079 & 12.698 & 9.523 & 9.920 & 10.317 & 7.936 \\
\hline$P_{5}^{1}$ & 25.000 & 13.095 & 19.841 & 16.666 & 19.047 & 4.365 & 21.825 \\
\hline$P_{6}^{1}$ & 34.127 & 13.888 & 14.285 & 12.698 & 13.492 & 10.317 & 7.936 \\
\hline$P_{7}^{1}$ & 26.587 & 22.619 & 13.492 & 13.095 & 20.634 & 11.507 & 11.507 \\
\hline$P_{8}^{1}$ & 25.000 & 20.238 & 9.126 & 13.095 & 33.333 & 9.523 & 5.952 \\
\hline$P_{9}^{1}$ & $\mathbf{6 7 . 8 5 7}$ & 27.381 & 11.111 & 12.698 & 26.984 & 28.571 & 11.111 \\
\hline$P_{10}^{1}$ & 40.079 & $\mathbf{5 8 . 3 3 3}$ & 13.888 & 15.873 & 33.333 & 9.126 & 6.746 \\
\hline$P_{11}^{1}$ & 13.888 & 8.333 & $\mathbf{6 3 . 0 9 5}$ & 17.063 & 6.349 & 5.158 & 18.650 \\
\hline$P_{12}^{1}$ & 20.238 & 11.507 & 26.190 & $\mathbf{7 0 . 6 3 4}$ & 12.301 & 6.746 & 30.555 \\
\hline$P_{13}^{1}$ & 30.952 & 28.968 & 7.936 & 11.904 & $\mathbf{5 5 . 1 5 8}$ & 28.571 & 3.968 \\
\hline$P_{14}^{1}$ & 32.936 & 34.523 & 7.936 & 5.952 & 25.000 & $\mathbf{6 2 . 6 9 8}$ & 5.555 \\
\hline$P_{15}^{1}$ & 10.317 & 12.301 & 16.269 & 32.142 & 13.492 & 9.523 & $\mathbf{7 4 . 6 0 3}$ \\
\hline
\end{tabular}

Tabla 3.4: (Continuación) Porcentajes de clasificación del sistema de verificación de los conjuntos $P_{j}^{1}$ con los modelos $M_{i}$.

\begin{tabular}{|c|c|c|c|}
\hline Modelo & Aciertos $P_{i}^{1}(\mathbf{\%})$ & Aciertos $P_{i}^{2}(\boldsymbol{\%})$ & Diferencia \\
\hline$M_{1}$ & 71.428 & 71.568 & 0.140 \\
\hline$M_{2}$ & 82.142 & 67.647 & -14.495 \\
\hline$M_{3}$ & 70.634 & 63.725 & -6.909 \\
\hline$M_{4}$ & 68.254 & 64.705 & -3.549 \\
\hline$M_{5}$ & 58.330 & 62.745 & 4.415 \\
\hline$M_{6}$ & 55.555 & 53.921 & -1.634 \\
\hline$M_{7}$ & 51.873 & 50.000 & -1.873 \\
\hline$M_{8}$ & 62.301 & 71.568 & 9.267 \\
\hline$M_{9}$ & 67.857 & 69.607 & 1.750 \\
\hline$M_{10}$ & 58.333 & 57.843 & -0.490 \\
\hline$M_{11}$ & 63.095 & 61.764 & -1.331 \\
\hline$M_{12}$ & 70.634 & 56.862 & -13.772 \\
\hline$M_{13}$ & 55.158 & 60.784 & 5.626 \\
\hline$M_{14}$ & 62.698 & 67.647 & 4.949 \\
\hline$M_{15}$ & 74.603 & 82.352 & 7.749 \\
\hline
\end{tabular}

Tabla 3.5: Variabilidad del clasificador con diferentes conjuntos de prueba. 
En este caso la clasificación se obtuvo únicamente operando el conjunto $P_{i}^{2}$ con el modelo $M_{i}$ respectivo, cuyo modelo presenta el mayor porcentaje de clasificación. En la tabla 3.5 se reportan los resultados de esta prueba. Se observa que los porcentajes de acierto disminuyen, al reducir el número de vectores del conjunto de prueba. Sin embargo, aún con esta tendencia, los porcentajes de acierto se mantienen por encima del $50 \%$ y en algunos casos son mayores con el conjunto $P_{i}^{2}$ que con $P_{i}^{1}$, este último de mayor cardinalidad. Estos resultados muestran que el sistema de verificación puede considerarse estable y sin dependencia de las frases pronunciadas. De esta forma los valores asignados a los parametros del núcleo, c y $\gamma$, permiten la operación adecuada del sistema. Adicionalmente se determina un tiempo estimado de 4 segundos de las muestras de voz que el sistema requiere para operar en tiempo real.

\subsection{Curvas DET}

Las curvas de comportamiento del error en la detección de usuarios (Detection Error Trade-off, DET), referidas como curvas DET, son un medio que permite observar el comportamiento del error de un sistema de verificación. El análisis se basa en determinar las probabilidades de los dos tipos de error que pueden suceder en el sistema.

Con base en la tabla 1.1, la cual se reproduce a continuación, podemos denotar las dos acciones posibles del sistema como $\mathbf{A}$ para aceptar y $\mathbf{R}$ para rechazar.

\begin{tabular}{|c|c|c|}
\hline$\cdot$ & \multicolumn{2}{|c|}{ Acción del Sistema } \\
\hline Propuesta & Acepta & Rechaza \\
\hline $\mathbf{H}$ & $\mathbf{B}$ & $E_{1}$ \\
\hline$\neg H$ & $E_{2}$ & $\mathbf{B}$ \\
\hline
\end{tabular}

Tabla 3.6: Definición lógica de los errores posibles en un sistema de verificación.

La hipótesis $\mathbf{H}=\{$ Muestra de voz de un usuario válido $\}$ y la negación de ésta $\neg H$, conforman un espacio de eventos dado por

$$
\Omega=\{(A, H),(R, H),(A, \neg H),(R, \neg H)\},
$$

de donde $(R, H)$ y $(A, \neg H)$ son los dos eventos considerados como error, entonces la probabilidad de error total está dada por

$$
p(\text { Error })=p(R, H)+p(A, \neg H),
$$

o de forma equivalente

$$
p(\text { Error })=p(R \mid H) p(H)+p(A \mid \neg H) p(\neg H) .
$$


A partir de la ecuación 3.7 se crea la función de costo de detección (Detection Cost Function, DCF):

$$
D C F=w_{1} p(R \mid H) p(H)+w_{2} p(A \mid \neg H) p(\neg H),
$$

donde $w_{1}$ y $w_{2}$ son pesos positivos que asignan un costo a cada tipo de error. Las curvas DET se generan a partir de la ecuación 3.8 al fijar $w_{1}, w_{2}, p(H)$ y $p(\neg H)$ y graficar $p(R \mid H)$ respecto de $p(A \mid \neg H)$. Los valores para estas variables son obtenidos a partir de los porcentajes de clasificación dados en las tablas 3.3 y 3.4. Estos porcentajes son separados en dos conjuntos, uno de ellos incluye los porcentajes del sistema para peticiones de usuarios válidos $\left(P_{i}, M_{i}\right)$, es decir los que se encuentran en la diagonal. De esta manera el conjunto consta de 15 valores y se denota por TrueScores. El segundo conjunto incluye los porcentajes del sistema para peticiones inválidas $\left(P_{j}, M_{i}, i \neq j\right)$, es decir los que no pertenecen a la diagonal. Este conjunto se denota por FalseScores e incluye 210 valores. Para mejorar la resolución de las curvas DET se incrementa la cardinalidad de TrueScores. Para ello dado $v_{i} \in$ TrueScores, $i=1, \ldots, 15$, se generán 500 valores con una distribución Normal, con media $v_{i}$ y varianza $\sigma^{2}$. De esta forma el conjunto final TrueScores consta de 7500 valores.

En las figuras 3.2, 3.3 y 3.4 se esquematizan los resultados para diferentes valores de $\sigma^{2}$.

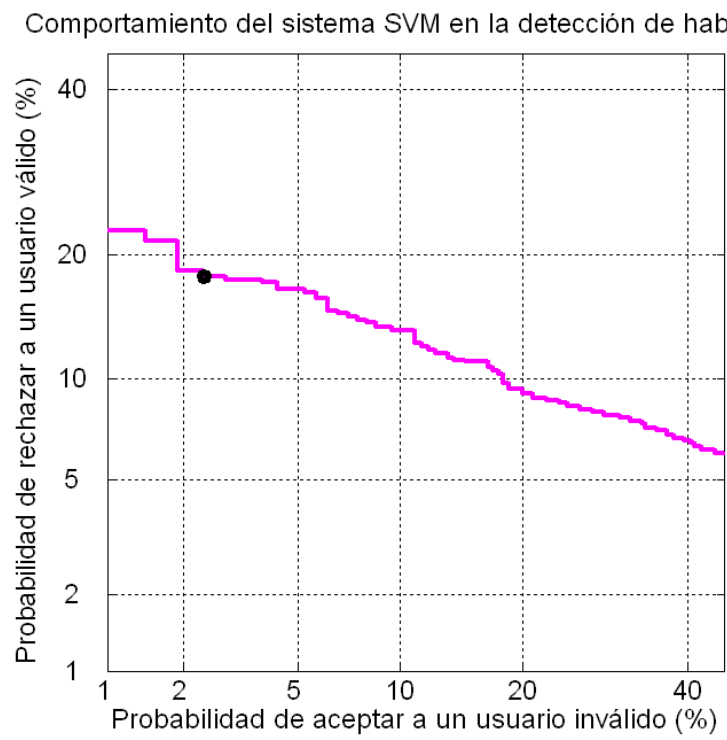

Figura 3.2: Curva DET del sistema de verificación con varianza $\sigma^{2}=10.0$ en los porcentajes de acierto.

La tabla 3.7 presenta los valores dados a los parámetros de la función DCF para obtener las curvas de desempeño del sistema. 


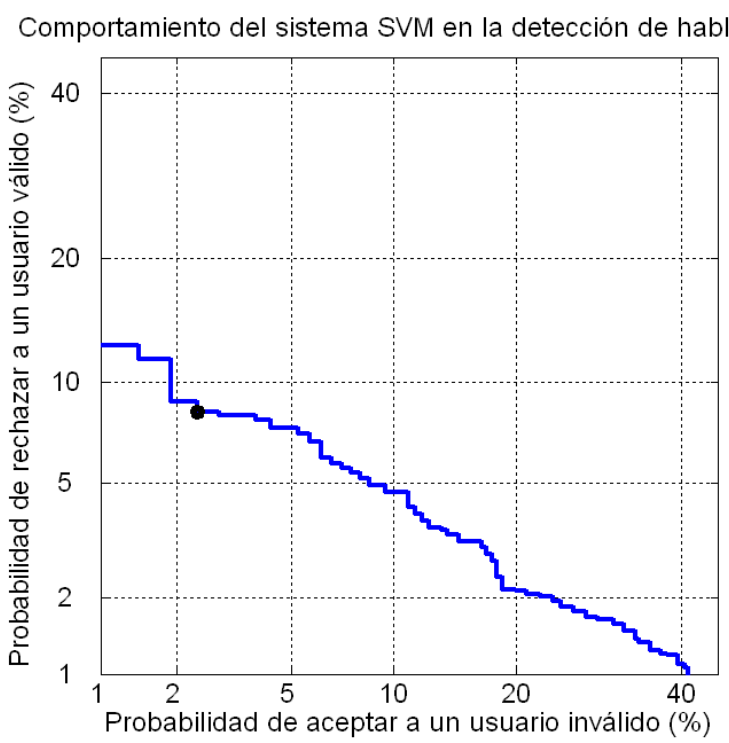

Figura 3.3: Curva DET del sistema de verificación con varianza $\sigma^{2}=4.0$ en los porcentajes de acierto.

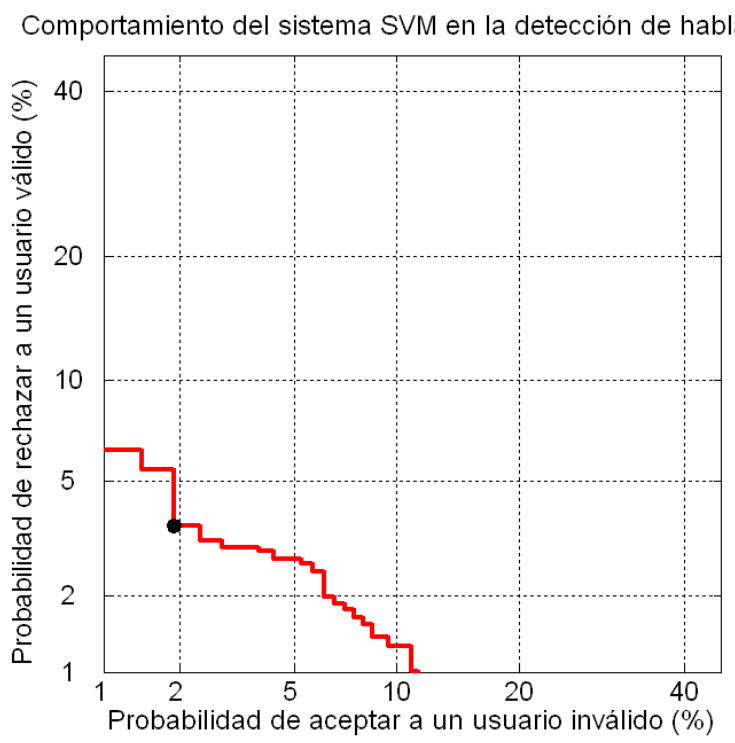

Figura 3.4: Curva DET del sistema de verificación con varianza $\sigma^{2}=2.0$ en los porcentajes de acierto. 


\begin{tabular}{|c|c|l|}
\hline Parámetro & Valor & Observaciones \\
\hline$p(H)$ & 0.015 & $\begin{array}{l}\text { Se tienen } 15 \text { usuarios válidos y un estimado total de } 1000 \\
\text { posibles. } p(H)=\frac{15}{1000} .\end{array}$ \\
\hline$p(\neg H)$ & 0.985 & $\begin{array}{l}\text { Se considera un total de 985 usuarios no válidos. } \\
p(\neg H)=1-p(H) .\end{array}$ \\
\hline$\left(w_{1}, w_{2}\right)$ & $(1,10)$ & $\begin{array}{l}\text { Se penaliza en una relación 10 a 1 el aceptar a un usuario } \\
\text { no válido. }\end{array}$ \\
\hline
\end{tabular}

Tabla 3.7: Parámetros asignados a la función DCF.

Es importante resaltar que los pesos asignados a $w_{1}$ y $w_{2}$ permiten reflejar condiciones reales de operacion del sistema. En este caso se considera que, por seguridad, es más grave la aceptación de un usuario inválido $(A \mid \neg H)$, que el rechazo de uno válido $(R \mid H)$.

Los valores óptimos encontrados por el algoritmo y que minimizan la función de costo DCF, son reportados en la tabla 3.8. Estos puntos también se encuentran graficados en las figuras $3.2,3.3$ y 3.4 .

\begin{tabular}{|c|c|c|c|c|c|}
\hline Curva & Varianza & $p(R \mid H)(\%)$ & $p(A \mid \neg H)(\%)$ & $p($ Error $)$ & Desempeño ( \%) \\
\hline fig. 3.2 & 10.0 & 17.773 & 2.381 & 0.0262 & 97.38 \\
\hline fig. 3.3 & 4.0 & 8.200 & 2.381 & 0.0247 & 97.53 \\
\hline fig. 3.4 & 2.0 & 3.506 & 1.905 & 0.0193 & 98.07 \\
\hline
\end{tabular}

Tabla 3.8: Valores óptimos de error que minimizan DCF y error y desempeño asociados.

El algoritmo utilizado para obtener las curvas DET, se encuentra especificado en el apéndice A.2. Éste consiste en obtener las distribuciones de TrueScores y FalseScores. La intersección entre ambas distribuciones constituye el error en la detección. Las escalas de graficación son tales, que una distribución normal aparace como una recta con pendiente -1 y distancia al origen determinada por la media y la varianza. Es posible entonces determinar un valor de error óptimo para el sistema de verificación, mediante la ecuación 3.7, y así también una medida de su desempeño, mediante la siguiente expresión:

$$
\text { Desempeño }=100(1-p(\text { Error }))=100(1-p(R \mid H) p(H)-p(A \mid \neg H) p(\neg H)) .
$$

Una descripción más detallada al respecto puede encontrarse en [2]. Esta medida nos permite observar que al disminuir la varianza en los porcentajes de acierto en la clasificación, se incrementa el desempeño del sistema. De esta forma se da un procedimiento de evaluación del sistema propuesto. 


\subsection{Comprobación con Voz en Tiempo Real}

Una parte determinante en la validación de un sistema de verificación, consiste en la realización de pruebas con voz en tiempo real. Esto debido a que, en última instancia, el sistema implementado operará con muestras de voz de un usuario, las cuales serán grabadas, procesadas y evaluadas por el sistema, hasta decidir la aceptación o rechazo del solicitante. La diferencia esencial entre las pruebas realizadas en la sección 3.1.2 y las que aquí se muestran, es que los conjuntos $E_{i}, B_{i}$, y $P_{i}, i=1, \ldots, 15$, son el resultado de una selección aleatoria de vectores del conjunto total $\left\{x_{i j}\right\}_{j=1}^{l}$, mientras que la comprobación en tiempo real utiliza muestras de secuencias de voz, es decir frases pronunciadas en forma secuencial y no necesariamente incluidas en el protocolo de grabación. Adicionalmente, éste tipo de pruebas permite evaluar el comportamiento, ante muestras de voz de hablantes que no cuenten con un modelo dentro del sistema de verificación. De esta forma se comprueba la independencia del sistema de las frases pronunciadas y se valida su operación ante peticiones de usuarios externos no válidos. En las secciones siguientes se presenta una descripción de las pruebas y resultados obtenidos para este fin, así como la evaluación del desempeño respectivo.

\subsubsection{Pruebas y Resultados}

La base de registros Voces-MCyTI se formó con 17 grabaciones de voz de 17 hablantes, de los cuales 15 de ellos fueron utilizados para la formación de modelos y la realización de pruebas, como se describió en la sección 3.2. Para la realización de pruebas, se seleccionó una muestra $R_{i}$ de cada uno de los 17 registros mcyti_i.wav. Las muestras $R_{i}, i=1, \ldots, 17$, se formaron extrayendo de cada registro, la tarea a) del protocolo de grabación (Sección 2.2.1), es decir el nombre completo del hablante. Dicha extracción se hizo de forma manual, de manera que el tiempo de duración de las muestras es variable, lo cual refleja una condición de operación real. La tabla 3.9 presenta las características de cada muestra $R_{i}$.

Una vez extraidas las muestras de voz, se sometieron al procesamiento referido en la sección 2.3 para extraer los vectores de características. Este procesamiento fue realizado en tres módulos: filtrado, supresión de silencios y obtención de vectores cepstrales. Con el objetivo de obtener un tiempo estimado requerido por el sistema para procesar las muestras $R_{i}$, se obtuvo el tiempo utilizado en cada uno de los módulos descritos. La tabla 3.10 presenta los tiempos de procesamiento de las muestras y también el número de vectores cepstrales obtenidos luego del procesamiento. Este número de vectores depende, principalmente, de la actividad del supresor de silencios, mismo que reduce la longitud de las muestras.

De esta manera se tiene que la duración promedio de las muestras $R_{i}$ es de $2,695 \mathrm{~s}$ y que, en promedio, para procesar dicho periodo de grabación se utilizan $\mathbf{5 , 4 0 6}$, esto sin considerar el tiempo utilizado por el clasificador. 


\begin{tabular}{|c|c|c|}
\hline Muestra & No. Puntos & Tiempo (s) \\
\hline$R_{1}$ & 19,573 & 2.446 \\
\hline$R_{2}$ & 19,774 & 2.471 \\
\hline$R_{3}$ & 19,828 & 2.478 \\
\hline$R_{4}$ & 19,771 & 2.471 \\
\hline$R_{5}$ & 19,996 & 2.499 \\
\hline$R_{6}$ & 20,005 & 2.500 \\
\hline$R_{7}$ & 19,355 & 2.419 \\
\hline$R_{8}$ & 19,674 & 2.459 \\
\hline$R_{9}$ & 35,841 & 4.480 \\
\hline$R_{10}$ & 23,840 & 2.980 \\
\hline$R_{11}$ & 19,963 & 2.495 \\
\hline$R_{12}$ & 20,030 & 2.503 \\
\hline$R_{13}$ & 20,761 & 2.595 \\
\hline$R_{14}$ & 19,971 & 2.496 \\
\hline$R_{15}$ & 19,976 & 2.497 \\
\hline$R_{16}$ & 25,142 & 3.142 \\
\hline$R_{17}$ & 23,147 & 2.893 \\
\hline
\end{tabular}

Tabla 3.9: Características de las muestras de voz $R_{i}$. 


\begin{tabular}{|c|c|c|c|c|c|}
\hline Muestra & t FIR (s) & t Supresor (s) & t MFCC (s) & Total (s) & Vectores \\
\hline$R_{1}$ & 3.594 & 0.359 & 0.218 & 4.171 & 56 \\
\hline$R_{2}$ & 3.704 & 0.375 & 0.250 & 4.329 & 77 \\
\hline$R_{3}$ & 3.703 & 0.344 & 0.266 & 4.313 & 85 \\
\hline$R_{4}$ & 3.688 & 0.375 & 0.187 & 4.250 & 59 \\
\hline$R_{5}$ & 5.422 & 0.360 & 0.266 & 6.048 & 85 \\
\hline$R_{6}$ & 4.016 & 0.375 & 0.203 & 4.594 & 68 \\
\hline$R_{7}$ & 5.484 & 0.375 & 0.125 & 5.984 & 39 \\
\hline$R_{8}$ & 5.359 & 0.265 & 0.094 & 5.718 & 29 \\
\hline$R_{9}$ & 6.641 & 0.437 & 0.297 & 7.375 & 92 \\
\hline$R_{10}$ & 4.609 & 0.438 & 0.172 & 5.219 & 58 \\
\hline$R_{11}$ & 5.484 & 0.266 & 0.297 & 6.047 & 94 \\
\hline$R_{12}$ & 3.735 & 0.218 & 0.125 & 4.078 & 36 \\
\hline$R_{13}$ & 4.187 & 0.391 & 0.296 & 4.874 & 97 \\
\hline$R_{14}$ & 5.422 & 0.375 & 0.156 & 5.953 & 51 \\
\hline$R_{15}$ & 5.609 & 0.453 & 0.219 & 6.281 & 71 \\
\hline$R_{16}$ & 4.844 & 0.469 & 0.188 & 5.501 & 59 \\
\hline$R_{17}$ & 6.422 & 0.500 & 0.250 & 7.172 & 81 \\
\hline
\end{tabular}

Tabla 3.10: Tiempos de procesamiento de las muestras $R_{i} \cdot c_{i}=10$ (Ver ecuación 2.6). 
La cantidad de vectores obtenidos varía en el intervalo de 29 a 97 . Este intervalo muestra una gran variabilidad y difiere respecto del número de vectores que conforman los conjuntos de prueba (252 y 102 vectores). Se conviene denotar al conjunto de vectores de características asociado a una muestra $R_{i}$ como $V_{R i}$. Con la finalidad de evaluar el comportamiento del sistema con muestras de voz en tiempo real, se procedió a clasificar cada uno de los conjuntos $V_{R i}, i=1, \ldots, 17$, con cada uno de los modelos $M_{j}, j=1, \ldots, 15$, generados mediante Máquinas de Soporte Vectorial y presentados en la sección 3.1.1, con el objetivo de obtener los porcentajes de clasificación respectivos. Estas pruebas tienen la siguiente interpretación:

Clasificación de $V_{R i}$ con $M_{i}, 1 \leq i \leq 15$. Se clasifica el conjunto de vectores de la muestra $R_{i}$, con el modelo de voz correspondiente al usuario $U_{i}$ del cual proviene. Esto significa que el sistema trata la petición de un usuario válido respecto de $M_{i}$, por lo que el porcentaje de acierto obtenido mide el desempeño o eficiencia del modelo generado para verificar al usuario con voz en tiempo real.

Clasificación de $V_{R i}$ con $M_{j}, i \neq j$. Se clasifica el conjunto de vectores de la muestra $R_{i}$, con el modelo de voz de otro usuario $U_{j}$, distinto. Esto significa que el sistema trata con una petición de un usuario inválido respecto de $M_{j}$, por lo que el porcentaje obtenido mide el error cometido por el sistema ante peticiones inválidas en tiempo real. Debido a que en este caso $i=1, \ldots, 17$ y $j=1, \ldots, 15$, los conjuntos $V_{R 16}$ y $V_{R 17}$ no cuentan con un modelo de voz $M_{i}$ dentro del sistema, lo cual significa que el sistema trata con peticiones de usuarios externos no incluidos.

Los resultados obtenidos de estas pruebas se encuentran reportados en las tablas 3.11 y 3.12 .

Los porcentajes obtenidos demuestran que las muestras de voz en tiempo real seleccionadas así como el número de vectores, son suficientes para la correcta operación del sistema, pues en cualquier caso siempre es posible determinar, de forma correcta y con base en el mayor porcentaje, a que modelo pertenecen las muestras de voz. Los porcentajes obtenidos al clasificar los conjuntos $V_{R 16}$ y $V_{R 17}$ con todos los modelos, muestran que el sistema opera adecuadamente en cuanto al rechazo de usuarios externos no incluidos, pues en ningún caso los porcentajes obtenidos superan el $50 \%$. De esta manera pueden inferirse dos reglas para la aceptación de un solicitante, que ingrese una muestra de voz $R$ al sistema:

1. Se selecciona el porcentaje de acierto con máximo valor obtenido, en la clasificación del conjunto de vectores de características asociado, $V_{R}$, con cada uno de los modelos $M_{j}, j=1, \ldots, 15$.

2. Se acepta al usuario con muestra de voz $R$, si el máximo porcentaje obtenido es mayor al $50 \%$. En caso contrario se rechaza. 


\begin{tabular}{|c|c|c|c|c|c|c|c|c|}
\hline$\cdot$ & $\mathbf{M}_{\mathbf{1}}$ & $\mathbf{M}_{\mathbf{2}}$ & $\mathbf{M}_{\mathbf{3}}$ & $\mathbf{M}_{\mathbf{4}}$ & $\mathbf{M}_{\mathbf{5}}$ & $\mathbf{M}_{\mathbf{6}}$ & $\mathbf{M}_{\mathbf{7}}$ & $\mathbf{M}_{\mathbf{8}}$ \\
\hline$V_{R 1}$ & $\mathbf{7 6 . 7 8 5}$ & 1.785 & 14.285 & 12.500 & 12.500 & 30.357 & 30.357 & 12.500 \\
\hline$V_{R 2}$ & 15.584 & $\mathbf{7 5 . 3 2 4}$ & 28.571 & 18.181 & 11.688 & 36.363 & 9.090 & 18.181 \\
\hline$V_{R 3}$ & 9.411 & 11.764 & $\mathbf{7 5 . 2 9 4}$ & 9.411 & 10.588 & 37.647 & 15.294 & 21.176 \\
\hline$V_{R 4}$ & 10.169 & 3.389 & 35.593 & $\mathbf{7 1 . 1 8 6}$ & 32.203 & 47.457 & 20.339 & 5.084 \\
\hline$V_{R 5}$ & 16.470 & 8.235 & 17.647 & 22.352 & $\mathbf{5 0 . 5 8 8}$ & 28.235 & 25.882 & 14.117 \\
\hline$V_{R 6}$ & 22.058 & 19.117 & 19.117 & 25.000 & 16.176 & $\mathbf{6 9 . 1 1 7}$ & 19.117 & 7.352 \\
\hline$V_{R 7}$ & 17.948 & 0.000 & 25.641 & 23.076 & 15.384 & 51.282 & $\mathbf{7 1 . 7 9 4}$ & 23.076 \\
\hline$V_{R 8}$ & 0.000 & 3.448 & 10.344 & 3.448 & 6.896 & 24.137 & 3.448 & $\mathbf{7 2 . 4 1 3}$ \\
\hline$V_{R 9}$ & 8.695 & 6.521 & 9.782 & 5.434 & 15.217 & 26.087 & 7.608 & 9.782 \\
\hline$V_{R 10}$ & 22.413 & 1.724 & 3.448 & 5.172 & 10.344 & 0.000 & 6.896 & 22.413 \\
\hline$V_{R 11}$ & 9.574 & 19.148 & 17.021 & 15.957 & 15.957 & 32.978 & 14.893 & 4.255 \\
\hline$V_{R 12}$ & 30.555 & 2.777 & 11.111 & 2.777 & 2.777 & 11.111 & 33.333 & 41.666 \\
\hline$V_{R 13}$ & 9.278 & 5.154 & 15.463 & 9.278 & 12.371 & 26.804 & 6.185 & 25.773 \\
\hline$V_{R 14}$ & 3.921 & 19.607 & 7.843 & 3.921 & 1.960 & 33.333 & 9.803 & 39.215 \\
\hline$V_{R 15}$ & 28.169 & 21.126 & 9.859 & 5.633 & 25.352 & 15.493 & 18.309 & 22.535 \\
\hline$V_{R 16}$ & 6.779 & 11.864 & 8.474 & 13.559 & 20.339 & 32.203 & 11.864 & 5.084 \\
\hline$V_{R 17}$ & 3.703 & 14.814 & 19.753 & 12.345 & 9.876 & 32.098 & 18.518 & 24.691 \\
\hline
\end{tabular}

Tabla 3.11: Comprobación del sistema SVM con muestras de voz en tiempo real. 


\begin{tabular}{|c|c|c|c|c|c|c|c|}
\hline$\cdot$ & $\mathbf{M}_{\mathbf{9}}$ & $\mathbf{M}_{\mathbf{1 0}}$ & $\mathbf{M}_{\mathbf{1 1}}$ & $\mathbf{M}_{\mathbf{1 2}}$ & $\mathbf{M}_{\mathbf{1 3}}$ & $\mathbf{M}_{\mathbf{1 4}}$ & $\mathbf{M}_{\mathbf{1 5}}$ \\
\hline$V_{R 1}$ & 10.714 & 12.500 & 37.500 & 37.500 & 25.000 & 5.357 & 32.142 \\
\hline$V_{R 2}$ & 20.779 & 14.285 & 19.480 & 18.181 & 7.792 & 7.792 & 16.883 \\
\hline$V_{R 3}$ & 8.235 & 12.941 & 11.764 & 12.941 & 12.941 & 15.294 & 22.352 \\
\hline$V_{R 4}$ & 15.254 & 11.864 & 3.389 & 5.084 & 11.864 & 23.728 & 3.389 \\
\hline$V_{R 5}$ & 31.764 & 17.647 & 16.470 & 20.000 & 27.058 & 5.882 & 18.823 \\
\hline$V_{R 6}$ & 26.470 & 16.176 & 13.235 & 4.411 & 17.647 & 8.823 & 5.882 \\
\hline$V_{R 7}$ & 33.333 & 25.641 & 20.512 & 17.948 & 30.769 & 20.512 & 10.256 \\
\hline$V_{R 8}$ & 17.241 & 13.793 & 3.448 & 13.793 & 65.517 & 17.241 & 0.000 \\
\hline$V_{R 9}$ & $\mathbf{8 5 . 8 6 9}$ & 8.695 & 6.521 & 15.217 & 39.130 & 34.782 & 14.130 \\
\hline$V_{R 10}$ & 41.379 & $\mathbf{7 2 . 4 1 3}$ & 8.620 & 13.793 & 31.034 & 15.517 & 6.896 \\
\hline$V_{R 11}$ & 10.638 & 9.574 & $\mathbf{7 3 . 4 0 4}$ & 13.829 & 12.766 & 8.510 & 11.702 \\
\hline$V_{R 12}$ & 11.111 & 5.555 & 16.666 & $\mathbf{7 7 . 7 7 7}$ & 13.888 & 5.555 & 41.666 \\
\hline$V_{R 13}$ & 35.051 & 25.773 & 7.216 & 19.587 & $\mathbf{7 1 . 1 3 4}$ & 27.835 & 6.185 \\
\hline$V_{R 14}$ & 19.607 & 45.098 & 11.764 & 7.843 & 21.568 & $\mathbf{8 0 . 3 9 2}$ & 11.764 \\
\hline$V_{R 15}$ & 8.450 & 18.309 & 28.169 & 46.478 & 15.493 & 4.225 & $\mathbf{7 3 . 2 3 9}$ \\
\hline$V_{R 16}$ & 5.084 & 0.000 & 28.813 & 16.949 & 18.644 & 1.694 & 25.423 \\
\hline$V_{R 17}$ & 12.345 & 16.049 & 12.345 & 12.345 & 44.444 & 16.049 & 14.814 \\
\hline
\end{tabular}

Tabla 3.12: (Continuación) Comprobación del sistema SVM con muestras de voz en tiempo real. 
Estas dos reglas establecen, de forma simple y con base en los porcentajes de clasificación obtenidos, un criterio de decisión para el sistema de verificación. Por supuesto que dicho criterio puede ser sujeto a comprobaciones más extensas.

\subsubsection{Curvas DET con Voz en Tiempo Real}

Como se hizo mención en la sección 3.2, las curvas DET son un instrumento que permite observar el comportamiento del error de un sistema, en este caso de verificación, con base en las distribuciones de porcentajes de acierto y error observados. Así los porcentajes de acierto, presentados en la diagonal de las tablas 3.11 y 3.12, conforman una distribución que es almacenada en un archivo llamado TrueScores. El resto de los valores de dichas tablas, conforman una distribución que es almacenada en el archivo FalseScores, por lo que este último conjunto consta de 230 valores. Nuevamente se mejora la resolución del conjunto TrueScores de la siguiente forma: para cada $v_{i} \in$ TrueScores, $i=1, \ldots, 15$, se genera un conjunto de 500 valores con distribución normal, con media $v_{i}$ y varianza $\sigma^{2}$. De esta forma el conjunto final TrueScores consta de 7500 valores. Las curvas DET son obtenidas a partir la función de costo dada en 3.7 y mediante el algoritmo dado en el apéndice A.2.

A modo de comparación, en las figuras 3.5, 3.6 y 3.7 se presenta el comportamiento del error del sistema SVM, mediante los conjuntos de prueba $P_{i}^{1}$ y mediante los conjuntos de vectores asociados a las muestras $R_{i}$, para tres valores diferentes de $\sigma^{2}$.

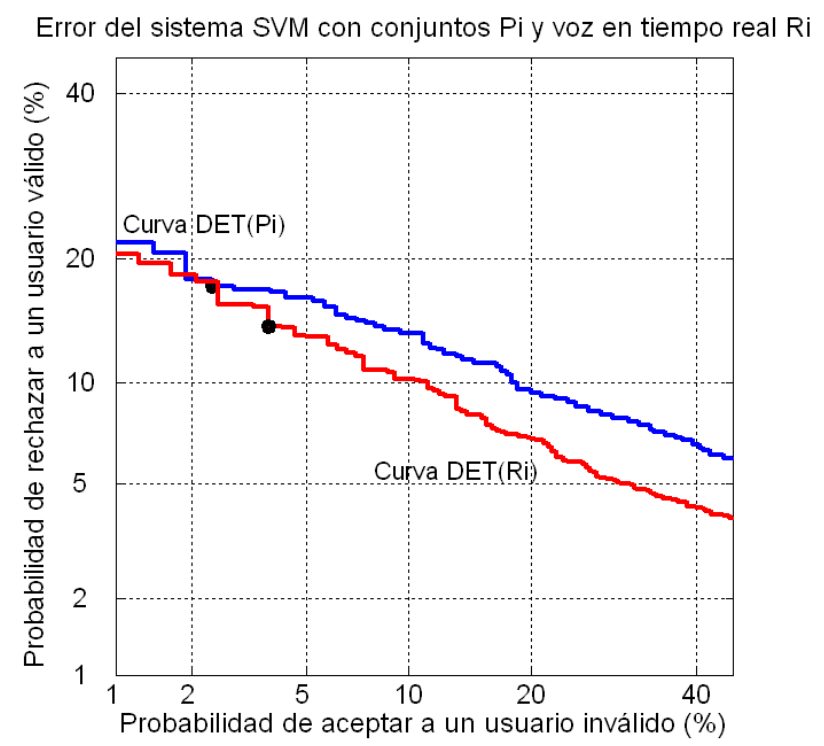

Figura 3.5: Comportamiento del error del sistema SVM con conjuntos de prueba $P_{i}^{1}$ y muestras de voz en tiempo real $R_{i} . \sigma^{2}=10.0$. 


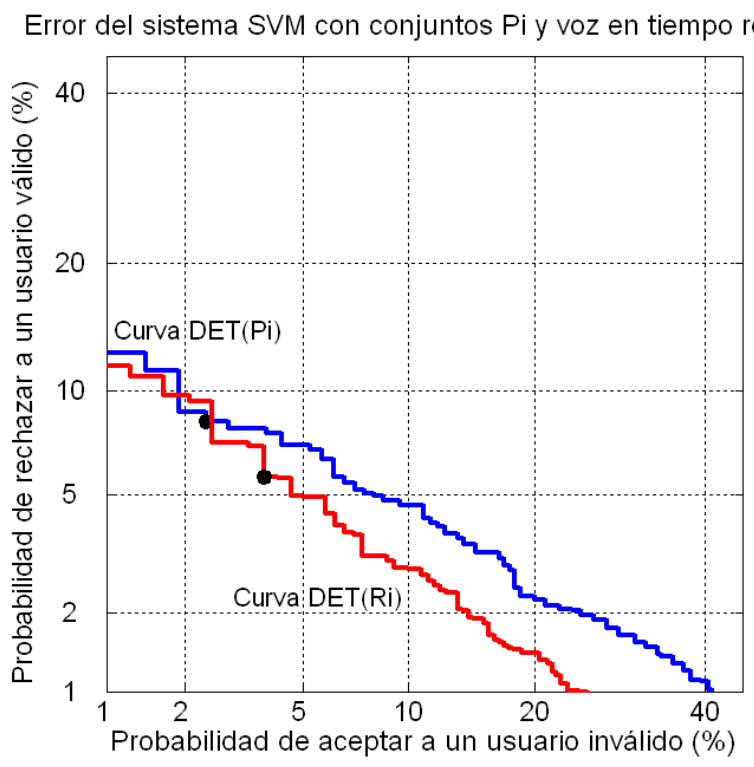

Figura 3.6: Comportamiento del error del sistema SVM con conjuntos de prueba $P_{i}^{1}$ y muestras de voz en tiempo real $R_{i} \cdot \sigma^{2}=4.0$.

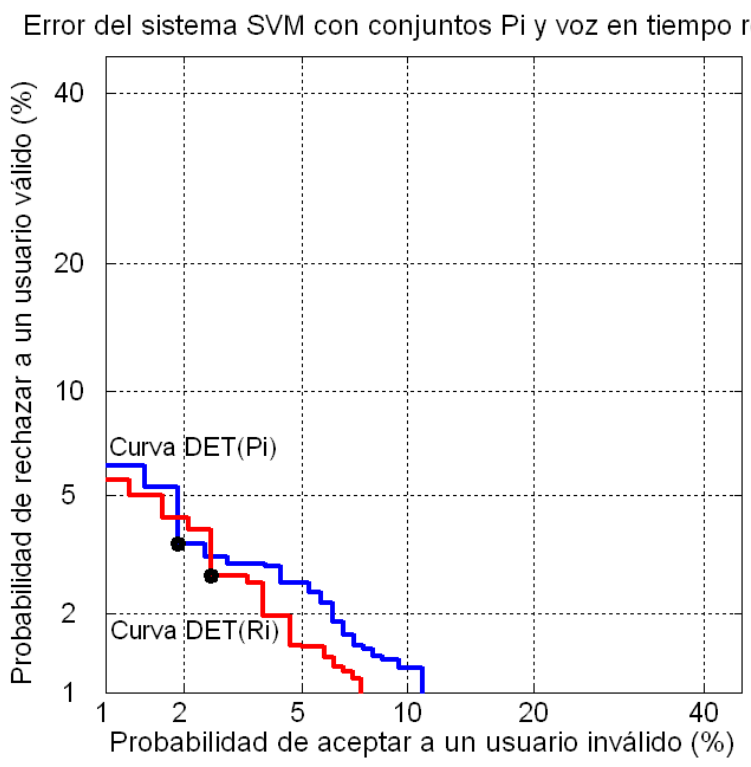

Figura 3.7: Comportamiento del error del sistema SVM con conjuntos de prueba $P_{i}^{1}$ y muestras de voz en tiempo real $R_{i} . \sigma^{2}=2.0$. 
Los parámetros asignados a la función DCF para la obtención de estas curvas, son los mismos que los reportados en la tabla 3.8. Los puntos óptimos de error encontrados y que minimizan DCF, son presentados en la tabla 3.13, donde también se presenta el desempeño asociado (Ecuación 3.9).

\begin{tabular}{|l|c|c|c|c|c|}
\hline Curva & Varianza & $p(R \mid H)(\%)$ & $p(A \mid \neg H)(\%)$ & $p($ Error $)$ & Desempeño ( \%) \\
\hline fig. 3.5 & 10.0 & 13.906 & 3.750 & 0.0390 & 96.10 \\
\hline fig. 3.6 & 4.0 & 5.680 & 3.750 & 0.0378 & 96.22 \\
\hline fig. 3.7 & 2.0 & 2.733 & 2.500 & 0.0250 & 97.50 \\
\hline
\end{tabular}

Tabla 3.13: Valores óptimos de error que minimizan DCF y error y desempeño asociados.

En la tabla 3.14 se comparan los valores de desempeño obtenidos en la tabla 3.8 con los que se reportan en la tabla 3.13 .

\begin{tabular}{|c|c|c|}
\hline$\cdot$ & \multicolumn{2}{|c|}{ Desempeño ( \%) } \\
\hline Varianza & $\mathbf{P}_{\mathbf{i}}^{\mathbf{1}}$ & $\mathbf{R}_{\mathbf{i}}$ \\
\hline 10.0 & 97.38 & 96.10 \\
\hline 4.0 & 97.53 & 96.22 \\
\hline 2.0 & 98.07 & 97.50 \\
\hline
\end{tabular}

Tabla 3.14: Comparación de desempeño del sistema SVM con conjuntos de prueba $P_{i}^{1}$ y voz en tiempo real $R_{i}$.

La tendencia observada en estos resultados y en las gráficas obtenidas, muestra que el desempeño del sistema con las muestras de voz $R_{i}$, es menor al presentado con los conjuntos de prueba $P_{i}^{1}$ en $1 \%$, esto aún cuando las gráficas $p(R \mid H)$ vs $P(A \mid \neg H)$ en tiempo real, muestran un mejor comportamiento. Las diferencias en los porcentajes mostrados en la tabla 3.14, permiten afirmar que el sistema SVM implementado es independiente del texto. También se valida que los modelos $M_{i}$, pueden operar con muestras de voz en tiempo real o con frases pronunciadas secuencialmente. Adicionalmente, se comprueba que este sistema tiene un comportamiento estable ante peticiones de usuarios que no cuentan con un modelo de voz.

\subsection{Resultados Recientes con SVM}

En esta sección se presentan resultados de investigación publicados recientemente en dos artículos. En éstos se utilizan las Máquinas de Soporte Vectorial como sistema de clasificación aplicado a tareas de verificación por voz. 
En ambos casos son presentados los detalles más relevantes de la implementación de forma sintética.

\section{1. "Speaker Verification Using Support Vector Machines" [45].}

Datos: Todas las pruebas realizadas y reportadas están basadas en la base de voz NIST 2003 Speaker Recognition Evaluation, en idioma inglés.

Método de procesamiento: Se utiliza la extracción de vectores mediante análisis MFCC normal o estándar. La dimensión de los vectores es 39. No se hace una descripción detallada del procedimiento de extracción.

Conjuntos de entrenamiento y prueba: Todas las muestras para formar los conjuntos fueron de aproximadamente 2 minutos de duración. El conjunto de entrenamiento se obtuvo a partir de 60 pronunciaciones, mientras que el conjunto de prueba se obtuvo de 78. Ambos conjuntos se extrajeron de la base Switchboard. Para el conjunto de vectores de entrenamiento de un usuario particular, se tomaron todos los vectores obtenidos mediante el análisis cepstral. Para conformar los vectores del resto de los usuarios (Background) se eligieron aleatoriamente $n$ vectores de cada conjunto de vectores de entrenamiento del resto de usuarios. El valor de $n$ fue elegido de manera que fuera el doble de vectores obtenido para cada usuario. Esta decisión fue tomada a fin de que la aplicación fuera factible, ya que se sabe que el desempeño de sistemas de clasificación mediante $S V M$ es adecuado sólo para conjuntos de entrenamiento pequeños.

Metodología de clasificación: Se aplica directamente a los vectores de características de la voz, la metodología de las Máquinas de Soporte Vectorial. Esta metodología ha sido expuesta ampliamente por lo que no se requiere una descripción más detallada.

Resultados: El primer paso en los experimentos fue obtener un valor óptimo para el parámetro $\gamma$ del núcleo $K$ (Ecuación 2.36). En este caso se obtuvo que no hay variación sustancial en los porcentajes para valores de $\gamma$ entre 0.02 y 2.5 y que el valor óptimo es de $\gamma=0.019$. El valor mínimo que se obtiene de la función DCF es de 0.1406. Adicionalmente se utilizan Modelos Ocultos de Markov con los que se reporta un valor mínimo de DCF de $\mathbf{0 . 2 1 2 4}$.

\section{2. "Support Vector Machines Using GMM Supervectors for Speaker Verifica-} tion" [54].

Datos: La base de datos usada fue 2005 NIST SRE. Esta base de voz fue grabada en inglés a partir de conversaciones telefónicas. La investigación se enfocó al uso de la grabación de entrenamiento No.8, individual y desde un mismo teléfono y al de 
la grabación de prueba No.1, individual y desde un mismo teléfono. En promedio el tiempo de conversacin es de 5 minutos con 2.5 minutos de voz. Este esquema permitió la formación de 1672 muestras asociadas a los usuarios y 14406 muestras utilizadas como Background.

Método de procesamiento: Se obtienen los coeficientes MFCC de dimensión 19 de la señal preenfatizada. Se utiliza segmentación en ventanas de tiempo de $20 \mathrm{~ms} \mathrm{y}$ un traslape temporal de 10ms con la ventana anterior. En estos segmentos se aplica una ventana de Hamming. Posteriormente se aplica la Transformada Discreta de Fourier y al espectro resultante un Banco Triangular de Filtros Mel. El espectro de la señal es limitado en un rango de $300 \mathrm{~Hz}$ a $3140 \mathrm{~Hz}$. Los vectores cepstrales son filtrados con $R A S T A$. Los coeficientes cepstrales delta son calculados considerando 2 vectores anteriores y dos posteriores al de cada trama y agregados al vector considerado con lo que se forma un vector de dimensión 38. Los vectores obtenidos son sometidos a un detector de energía que descarta vectores con baja intensidad (silencios). Se aplica por último un mapeo de vectores para remover los efectos del canal y normalizar según la media y la varianza a cada vector.

Conjuntos de entrenamiento y prueba: El modelo para el resto de los usuarios, consiste en una Mezcla Gaussiana de 2048 componentes (Gaussian Mixture Models, GMM, véase sección 4.1). Para el entrenamiento se adaptó la media con un factor de relevancia de 16. El proceso de entrenamiento se llevó a cabo con el algoritmo Expectation-Maximization EM, con datos obtenidos de las siguientes bases: Switchboard 2 fase 1, Switchboard 2 fase 4 (celular) y OGI National Cellular. Se produjeron 2,326 supervectores GMM para formar el Background etiquetados con -1. Para el enrolado de usuarios se produjeron 8 supervectores GMM por cada uno, provenientes de 8 conversaciones. El entrenamiento de la Máquina de Soporte Vectorial se realizó con los 8 supervectores de cada usuario y el Background.

Metodología de clasificación: El método consiste en la generación de 8 supervectores provenientes de un Modelo de Mezclas de funciones de Gauss, a partir de la media conjunta de los componentes de la mezcla. Este supervector es usado para caracterizar al hablante y al canal mediante el uso de eigenvoces y eigencanales, respectivamente. También se extraen 2,326 supervectores para el Background. La Máquina de Soporte Vectorial clasifica a estos supervectores y genera el modelo final para cada hablante. Dos funciones núcleo son empleadas para llevar a cabo la clasificación.

Resultados: Dos de los cuatro esquemas de pruebas presentados utilizaron SVM: Supervetores GMM y clasificación SVM usando un núcleo lineal y supervectores GMM y clasificación SVM usando un núcleo no lineal. Los mejores resultados de clasificación se obtuvieron con el núcleo lineal con un valor óptimo de DCF de 0.0139 . 
A modo de síntesis se presentan en la tabla 3.15 los valores de desempeño obtenidos de las aplicaciónes SVM descritas anteriormente.

\begin{tabular}{|c|c|c|}
\hline Sistema & minDCF & Desempeño \\
\hline SVM [45] & 0.1406 & $85.94 \%$ \\
\hline GMM-SVM [54] & 0.0139 & $98.61 \%$ \\
\hline SVM propuesto & 0.0193 & $98.07 \%$ \\
\hline
\end{tabular}

Tabla 3.15: Comparación de desempeño entre dos sistemas SVM publicados recientemente (2006) y el propuesto en este trabajo.

Es importante resaltar que el desempeño obtenido en el sistema SVM desarrollado en este trabajo, es muy próximo al obtenido mediante una técnica más elaborada como la que se presenta en [54].

\subsection{Validación del Supresor de Silencios}

La supresión de silencios se presentó como parte importante del procesamiento de la señal previo a la obtención de los vectores de características (sección 2.3.1). En esta sección se presentan las pruebas y resultados que validan y justifican su aplicación.

Se debe considerar que las pruebas y resultados de desempeño reportadas previamente en este capítulo, fueron realizadas a partir de vectores extraidos de señales sometidas al supresor de silencios. Por ello, para establecer una validación es necesario clasificar conjuntos de vectores provenientes de señales que no hayan sido sometidas a este procesamiento. Por simplicidad se eligieron las muestras de voz $R_{i}$ presentadas en la sección 3.3, que incluyen el nombre del hablante. De esta forma los resultados que se obtengan pueden ser comparados con los correspondientes. Ello permitirá formular conclusiones sobre los efectos de aplicar el supresor de silencios propuesto.

Las pruebas consistieron en procesar los registros de voz, excluyendo la etapa de supresión de silencios, y clasificar cada conjunto de vectores denotado por $R s_{i}$, con cada uno de los modelos $M_{i}$ creados en la sección 3.1.1. Los resultados de la clasificación son presentados en la tablas 3.16 y 3.17. Los mayores porcentajes de clasificación son resaltados en "negritas" para cada uno de los conjuntos de prueba.

De los resultados obtenidos en ambas tablas pueden establecerse los siguientes puntos que validan la etapa de supresión de silencios propuesta: 


\begin{tabular}{|c|c|c|c|c|c|c|c|c|}
\hline$\cdot$ & $\mathbf{M}_{\mathbf{1}}$ & $\mathbf{M}_{\mathbf{2}}$ & $\mathbf{M}_{\mathbf{3}}$ & $\mathbf{M}_{\mathbf{4}}$ & $\mathbf{M}_{\mathbf{5}}$ & $\mathbf{M}_{\mathbf{6}}$ & $\mathbf{M}_{\mathbf{7}}$ & $\mathbf{M}_{\mathbf{8}}$ \\
\hline$R s_{1}$ & $\mathbf{6 0 . 8 3 3}$ & 3.333 & 26.666 & 20.000 & 15.000 & 27.500 & 34.166 & 7.500 \\
\hline$R s_{2}$ & 12.396 & $\mathbf{6 3 . 6 3 6}$ & 23.966 & 19.834 & 11.570 & 39.669 & 18.181 & 18.181 \\
\hline$R s_{3}$ & 12.295 & 12.295 & $\mathbf{6 8 . 0 3 2}$ & 13.114 & 9.016 & 45.082 & 17.213 & 20.491 \\
\hline$R s_{4}$ & 9.016 & 5.737 & 31.967 & 40.163 & 28.688 & $\mathbf{5 0 . 0 0 0}$ & 18.032 & 7.377 \\
\hline$R s_{5}$ & 22.764 & 12.195 & 23.577 & 22.764 & $\mathbf{5 0 . 4 0 6}$ & 40.650 & 20.325 & 11.382 \\
\hline$R s_{6}$ & 33.333 & 14.634 & 31.707 & 21.951 & 28.455 & $\mathbf{6 5 . 0 4 0}$ & 14.634 & 11.382 \\
\hline$R s_{7}$ & 15.966 & 11.764 & 31.092 & 13.445 & 17.647 & $\mathbf{5 2 . 1 0 0}$ & 41.176 & 13.445 \\
\hline$R s_{8}$ & 11.570 & 7.438 & 23.140 & 9.090 & 9.090 & 28.925 & 17.355 & 33.057 \\
\hline$R s_{9}$ & 6.306 & 16.216 & 26.126 & 11.261 & 30.630 & 47.747 & 6.306 & 4.954 \\
\hline$R s_{10}$ & 18.367 & 12.244 & 19.047 & 10.884 & 7.482 & 5.442 & 13.605 & 13.605 \\
\hline$R s_{11}$ & 8.130 & 17.073 & 21.951 & 21.138 & 21.951 & 35.772 & 20.325 & 9.756 \\
\hline$R s_{12}$ & 15.447 & 7.317 & 13.008 & 6.504 & 14.634 & 32.520 & 19.512 & 13.821 \\
\hline$R s_{13}$ & 4.687 & 10.937 & 14.843 & 10.156 & 16.406 & 32.031 & 14.062 & 19.531 \\
\hline$R s_{14}$ & 4.065 & 13.821 & 8.130 & 12.195 & 9.756 & 39.024 & 18.699 & 38.211 \\
\hline$R s_{15}$ & 17.073 & 17.886 & 8.943 & 2.439 & 12.195 & 31.703 & 16.260 & 26.016 \\
\hline$R s_{16}$ & 8.387 & 24.516 & 10.322 & 18.709 & 20.645 & $\mathbf{2 9 . 0 3 2}$ & 16.129 & 3.870 \\
\hline$R s_{17}$ & 4.895 & 6.293 & 12.587 & 27.272 & 18.881 & $\mathbf{3 9 . 1 6 0}$ & 10.489 & 16.083 \\
\hline
\end{tabular}

Tabla 3.16: Porcentajes de clasificación mediante SVM con muestras de voz $R_{i}$ procesadas sin suprimir silencios. 


\begin{tabular}{|c|c|c|c|c|c|c|c|}
\hline$\cdot$ & $\mathbf{M}_{\mathbf{9}}$ & $\mathbf{M}_{\mathbf{1 0}}$ & $\mathbf{M}_{\mathbf{1 1}}$ & $\mathbf{M}_{\mathbf{1 2}}$ & $\mathbf{M}_{\mathbf{1 3}}$ & $\mathbf{M}_{\mathbf{1 4}}$ & $\mathbf{M}_{\mathbf{1 5}}$ \\
\hline$R s_{1}$ & 25.833 & 21.666 & 23.333 & 24.166 & 14.166 & 2.500 & 24.166 \\
\hline$R s_{2}$ & 30.578 & 15.702 & 17.355 & 10.743 & 9.917 & 8.264 & 10.743 \\
\hline$R s_{3}$ & 12.295 & 18.852 & 10.655 & 12.951 & 11.475 & 9.836 & 10.852 \\
\hline$R s_{4}$ & 25.409 & 16.393 & 16.393 & 4.098 & 10.655 & 19.672 & 8.196 \\
\hline$R s_{5}$ & 36.585 & 15.447 & 16.260 & 13.821 & 21.138 & 7.317 & 15.447 \\
\hline$R s_{6}$ & 34.959 & 10.569 & 13.821 & 4.878 & 8.943 & 6.504 & 8.943 \\
\hline$R s_{7}$ & 31.092 & 18.487 & 21.008 & 15.126 & 10.084 & 10.084 & 12.605 \\
\hline$R s_{8}$ & $\mathbf{3 7 . 1 9 0}$ & 23.966 & 10.743 & 14.876 & 27.272 & 12.396 & 9.090 \\
\hline$R s_{9}$ & $\mathbf{5 9 . 0 0 9}$ & 17.567 & 10.810 & 20.720 & 26.126 & 27.027 & 12.162 \\
\hline$R s_{10}$ & 47.619 & $\mathbf{5 3 . 0 6 1}$ & 14.966 & 8.163 & 27.210 & 11.564 & 5.442 \\
\hline$R s_{11}$ & 13.008 & 15.447 & $\mathbf{5 6 . 9 1 0}$ & 17.886 & 12.195 & 7.317 & 13.008 \\
\hline$R s_{12}$ & 30.081 & 8.943 & 27.642 & $\mathbf{6 5 . 8 5 3}$ & 17.073 & 4.878 & 35.772 \\
\hline$R s_{13}$ & 31.250 & 21.093 & 7.812 & 16.406 & $\mathbf{5 7 . 8 1 2}$ & 39.843 & 7.031 \\
\hline$R s_{14}$ & 22.764 & 34.959 & 22.764 & 8.943 & 24.390 & $\mathbf{6 0 . 1 6 2}$ & 8.943 \\
\hline$R s_{15}$ & 24.390 & 17.886 & 17.886 & 35.772 & 20.325 & 16.260 & $\mathbf{4 4 . 7 1 5}$ \\
\hline$R s_{16}$ & 14.838 & 10.967 & 23.871 & 15.483 & 20.645 & 3.870 & 26.451 \\
\hline$R s_{17}$ & 16.083 & 9.090 & 12.587 & 6.993 & 25.174 & 23.076 & 11.888 \\
\hline
\end{tabular}

Tabla 3.17: (Continuación) Porcentajes de clasificación mediante SVM con muestras de voz $R_{i}$ procesadas sin suprimir silencios. 
1. Los porcentajes más altos en la clasificación para $R s_{4}$ y $R s_{7}$ son con el modelo $M_{6}$.

2. El mejor porcentaje de clasificación para $R s_{8}$ se obtiene con el modelo $M_{9}$.

3. Con base en los puntos 1 y 2 los modelos $M_{4}, M_{7}$ y $M_{8}$ quedan sin asignación de porcentaje máximo. En una tarea de reconocimiento esto implicaría la aceptación de un usario válido a través de una verificación erronea.

4. Debido a que el mejor porcentaje de acierto para $R s_{8}$ es de 37.190 , el porcentaje para rechazar un usuario debería estar por debajo de este nivel. Bajo este criterio el usuario $R s_{17}$ se asignaría al modelo $M_{6}$ lo que implicaría la aceptación de un usario inválido.

5. Bajo los criterios de decisión derivados en la sección 3.3.1 para aceptación o rechazo de hablantes (pag. 62), se hace patente el rechazo de usuarios válidos, como sucedería con $R s_{8}$ y $R s_{15}$.

6. Comparando los porcentajes obtenidos con los de las tablas 3.11 y 3.12 correspondientes, se concluye que los valores TrueScores se reducen hasta en 39 puntos $\left(R s_{8}\right.$ y $\left.M_{8}\right)$, mientras que los correspondientes a FalseScores se incrementan hasta en 11 puntos $\left(R s_{6}\right.$ y $\left.M_{1}\right)$.

7. Se infiere que las distribuciones para TrueScores y FalseScores son más próximas, es decir se disminuye la resolución entre ambos conjuntos.

De esta forma se valida la etapa de supresión de silencios, pues al omitirla se demuestra el incremento de las probabilidades de error del sistema e incluso su disfuncionalidad. Debido a que se presenta rechazo de usuarios válidos y aceptación de usuarios inválidos (errores de tipo I y II), se infiere un comportamiento insuficiente para implementar un sistema de verificación y la necesidad de contar con una etapa que suprima los silencios de los registros de voz. 


\section{Capítulo 4}

\section{Otros Sistemas de Clasificación}

En este capítulo se presentan dos sistemas de clasificación adicionales, mismos que fueron usados para comparar el desempeño obtenido mediante Máquinas de Soporte Vectorial. Para ello se utilizaron los mismos conjuntos $E_{i}$ y $P_{i}^{1}$, descritos en el capítulo 3 para la generación de modelos y la realización de pruebas. El procedimiento utilizado para obtener el desempeño de los sistemas propuestos fue expuesto en la sección 3.2. En las secciones siguientes se presenta una descripción breve de cada sistema, así como los resultados de clasificación al aplicarlos a la base de registros Voces-MCyTI.

\subsection{Modelos de Mezclas Gaussianas}

Una mezcla de Gaussianas o mezcla de funciones de Gauss (Gaussian Mixture Models, $G M M)$ para un modelo $\lambda_{i}$, es una combinación de funciones de probabilidad denotada por

$$
p\left(x \mid \lambda_{i}\right)=\sum_{j=1}^{M_{i}} w_{i j} p_{i j}(x),
$$

donde $x \in \mathbb{R}^{n}, p_{i j}$ es una función de probabilidad de Gauss en $\mathbb{R}^{n}$, con media $\mu_{i j} \in \mathbb{R}^{n}$ y matriz de covarianzas $\Sigma_{i j} \in \mathbb{R}^{n} \times \mathbb{R}^{n} . w_{i j} \in \mathbb{R}, j=1 \ldots M_{i}$, es un conjunto de valores positivos que satisface

$$
\sum_{j=1}^{M_{i}} w_{i j}=1
$$

De esta forma se obtiene que la función 4.1 es también una función de probabilidad. Así, se puede denotar a cada una de las funciones $p_{i j}$ como 


$$
p_{i j}(x)=\frac{1}{(2 \pi)^{\frac{n}{2}}\left|\Sigma_{i j}\right|^{1 / 2}} \exp \left(-\frac{1}{2}\left(x-\mu_{i j}\right) \Sigma_{i j}^{-1}\left(x-\mu_{i j}\right)^{T}\right), j=1 \ldots M_{i} .
$$

Por lo anterior el modelo $\lambda_{i}$ queda determinado por

$$
\lambda_{i}=\left(\left\{w_{i j}\right\},\left\{\mu_{i j}\right\},\left\{\Sigma_{i j}\right\}, M_{i}\right) .
$$

La función 4.1 puede ser empleada para implementar un sistema de clasificación para verificación por voz. Si suponemos la existencia de conjuntos de vectores de características para entrenamiento y de Background, denotados por $E_{i}$ y $B_{i}$ respectivamente, correspondientes a un usuario $U_{i}$, entonces es posible generar un modelo $\lambda_{i}$ asociado a $E_{i}$ y un modelo $\lambda_{B i}$ asociado a $B_{i}$. Cada uno de estos modelos se obtiene ajustando los valores dados en 4.4, de forma que se maximice la función 4.1, para cada uno de los vectores del conjunto considerado. El algoritmo utilizado para este fin es denotado por $E M$, por sus siglas en inglés Expectation Maximization. Este algoritmo incrementa de forma monótona el valor de probabilidad de la función 4.1, para cada uno de los vectores de entrenamiento (de $E_{i}$ ó $B_{i}$ ) en cada iteración [14]. Las ecuaciones del algoritmo EM pueden ser encontradas en [13].

Una vez obtenidos los modelos $\lambda_{i}$ y $\lambda_{B i}$ de un usuario se obtienen también los valores de probabilidad asociados a dichos modelos así como su media y su varianza. Dado un conjunto de prueba $P_{i}$, se pueden validar dichos modelos, al determinar la pertenencia de cada $x \in P_{i}$ al modelo al que muestre una mayor proximidad, en términos de sus parámetros de media y varianza. Dado que se sabe a priori si $x$ proviene de un usuario válido o de uno inválido, se pueden determinar porcentajes de error ante ambos tipos de peticiones.

Es importante precisar que existen condiciones que afectan la representatividad de $B_{i}$, principalmente porque $B_{i}$ proviene de un subconjunto propio del conjunto complemento $\left\{U_{i}\right\}^{c}$, es decir $B_{i}$ no representa al total de los hablantes distintos a $U_{i}$. Este hecho, esquematizado en la figura 4.1, significa que $x \notin E_{i}$ no necesariamente implica que $x \in B_{i}$. De forma análoga $x \notin B_{i}$ no necesariamente implica que $x \in E_{i}$ y viceversa.

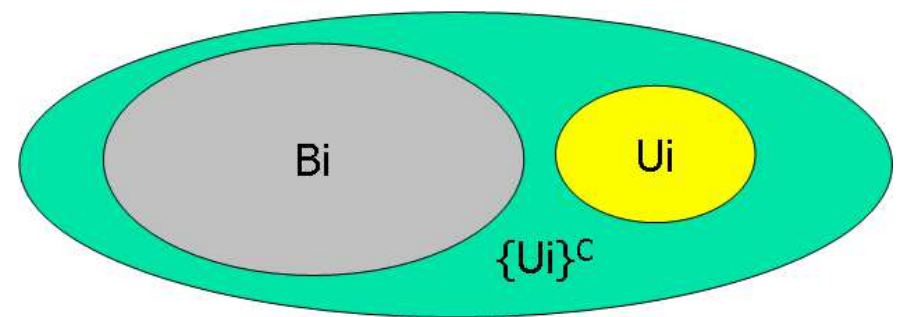

Figura 4.1: Representatividad del Background de un usuario respecto del universo de hablantes. 
Si denotamos por $\neg \lambda_{i}$ al modelo del resto de los usuarios a $U_{i}$ se tiene el siguiente cociente:

$$
\frac{p\left(x \mid \lambda_{i}\right)}{p\left(x \mid \neg \lambda_{i}\right)}
$$

al que se denomina Cociente de Probabilidades y es denotado por LR por sus siglas en inglés Likelihood Ratio. Debido a que el modelo $\lambda_{i}$ se obtiene maximizando $p\left(x \mid \lambda_{i}\right)$ para un conjunto de vectores $x \in E_{i}$, la función $p\left(x \mid \neg \lambda_{i}\right)$ es entonces minimizada. De esta manera el cociente LR toma también un valor máximo para el conjunto $E_{i}$, el cual puede puede denotarse por

$$
L R_{\max }\left(E_{i}\right)
$$

Mediante el cociente LR y un valor de umbral $\theta_{i}$ es posible determinar si un vector $x$ corresponde al usuario $U_{i}$ o no y así obtener porcentajes de acierto y error:

$$
L R(x)=\frac{p\left(x \mid \lambda_{i}\right)}{p\left(x \mid \neg \lambda_{i}\right)} \begin{cases}\geq \theta_{i} & x \text { pertenece a } \lambda_{i} . \\ <\theta_{i} & x \text { pertenece a } \neg \lambda_{i} .\end{cases}
$$

El establecimiento de un valor de umbral $\theta_{i}$ es una labor experimental que depende de los conjuntos de entrenamiento y prueba utilizados, pues su valor depende directamente de $L R_{\text {max }}\left(E_{i}\right)$, es decir que

$$
0 \leq \theta_{i}<R_{\max }\left(E_{i}\right)
$$

Es común que se utilice el logaritmo natural del cociente LR en lugar de este último [14]:

$$
\log (L R(x))=\log \left(\frac{p\left(x \mid \lambda_{i}\right)}{p\left(x \mid \neg \lambda_{i}\right)}\right)=\log p\left(x \mid \lambda_{i}\right)-\log p\left(x \mid \neg \lambda_{i}\right),
$$

el cual se denota por LLR por sus siglas en inglés Logarithmic Likelihood Ratio. Así, es común la programación de algoritmos EM utilizando el cociente LLR, por ejemplo el desarrollado por el IDIAP Research Institute llamado Torch (http://www.idiap.ch). Este software permite obtener, para un conjunto de vectores dado, el valor de $-\log \left(L R_{\max }\right)$ referido en 4.6. Para el caso de clasificación se generan dos modelos; $\lambda_{i}$ y $\lambda_{B_{i}}$ correspondientes a los conjuntos $E_{i}$, del usuario $U_{i}$, y $B_{i}$, que representa el resto de los usuarios a $U_{i}$, respectivamente. De esta forma, dado un conjunto de prueba $P_{i}$ y un vector $x \in P_{i}$ del cual se conoce su origen, se pueden obtener los siguientes valores:

$$
\begin{aligned}
-L L R_{\lambda_{i}}(x) & =-\log \left(\frac{p\left(x \mid \lambda_{i}\right)}{p\left(x \mid \neg \lambda_{i}\right)}\right), \\
-L L R_{\lambda_{B i}}(x) & =-\log \left(\frac{p\left(x \mid \lambda_{B i}\right)}{p\left(x \mid \neg \lambda_{B i}\right)}\right) .
\end{aligned}
$$


Para determinar, con base en las ecuaciones 4.10 y 4.11, a cual de los dos modelos pertenece $x$ con mayor probabilidad, es suficiente elegir el modelo cuyo valor de $L L R(x)$ sea mayor. Supongamos por ejemplo que

$$
L L R_{\lambda_{i}}(x)>L L R_{\lambda_{B i}}(x),
$$

entonces se da el siguiente desarrollo:

$$
\begin{gathered}
\log \left(\frac{p\left(x \mid \lambda_{i}\right)}{p\left(x \mid \lambda_{i}\right)}\right)>\log \left(\frac{p\left(x \mid \lambda_{B i}\right)}{p\left(x \mid \neg \lambda_{B i}\right)}\right) \Rightarrow \\
\frac{p\left(x \mid \lambda_{i}\right)}{p\left(x \mid \neg \lambda_{i}\right)}>\frac{p\left(x \mid \lambda_{B i}\right)}{p\left(x \mid \neg \lambda_{B i}\right)} \Rightarrow \\
p\left(x \mid \lambda_{i}\right) p\left(x \mid \neg \lambda_{B i}\right)>p\left(x \mid \lambda_{B i}\right) p\left(x \mid \neg \lambda_{i}\right) .
\end{gathered}
$$

La desigualdad 4.13, significa que es más probable que $x$ pertenezca a $\lambda_{i}$ y no pertenezca a $\lambda_{B i}$, a que pertenezca a $\lambda_{B i}$ y no pertenezca a $\lambda_{i}$. De este silogismo se deduce que $x$ corresponde al usuario $U_{i}$ con mayor probabilidad y se considera como una petición válida. $\mathrm{Al}$ invertir la desigualdad en 4.12 se obtiene, de manera análoga, que $x$ corresponde al complemento del usuario $U_{i}$ con mayor probabilidad y es por ello considerado como una petición inválida.

De esta forma se establece el uso de Modelos de Mezclas de Gaussianas como un sistema de clasificación y el procedimiento para la validación de los modelos generados.

\subsubsection{Pruebas y Resultados}

Para las pruebas con GMM se consideraron los mismos conjuntos de vectores $E_{i}, B_{i}$ y $P_{i}^{1}$ descritos en el capítulo 3 y que corresponden a cada uno de los 15 usuarios $U_{i}$ de la base Voces-MCyTI. Los conjuntos $E_{i}$ y $B_{i}$ fueron utilizados para generar los modelos $\lambda_{i}$ del usuario $U_{i}$ y del Background del usuario $\lambda_{B i}$, respectivamente. Los parámetros asignados a Torch para la generación de modelos se muestran en la tabla 4.1.

\begin{tabular}{|c|c|}
\hline Parámetro & Valor \\
\hline Número de Gaussianas & 10 \\
\hline Iteraciones matriz de covarianzas & 25 \\
\hline Iteraciones vector de promedios & 25 \\
\hline Normalización de datos & No \\
\hline
\end{tabular}

Tabla 4.1: Parámetros asignados para la generación de modelos con GMM. 
Estos parámetros fueron establecidos luego de realizar pruebas para obtener mejores porcentajes de acierto en la clasificación. El número de iteraciones referido en la tabla 4.1 es un valor máximo. Al incrementar estos valores no se observa una mejoría en los porcentajes de acierto. También se observó que la normalización de los conjuntos de vectores no incrementa los porcentajes de acierto, sino al contrario, por lo que dichos conjuntos fueron usados sin normalización.

El procedimiento seguido para evaluar un conjunto de vectores de prueba $P_{j}$ respecto de los modelos $\lambda_{i}$ y $\lambda_{B i}$ y obtener porcentajes de acierto, se encuentra esquematizado en la figura 4.2 .

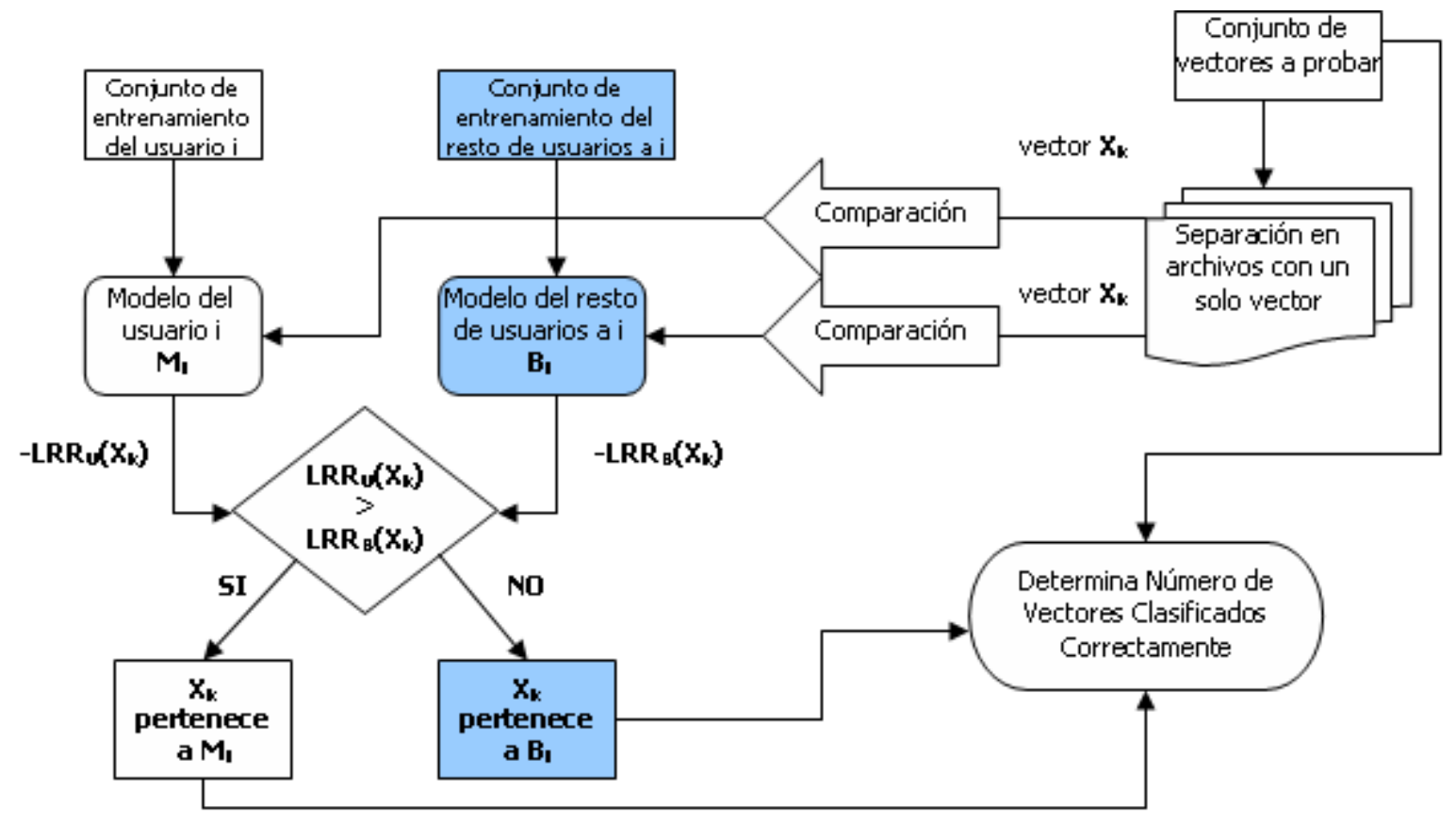

Figura 4.2: Procedimiento para obtención de porcentajes de acierto con GMM.

Con base en este procedimiento se realizaron pruebas al sistema de verificación basado en GMM. Para ello se sometió cada conjunto de prueba $P_{i}^{1}, i=1, \ldots, 15$, a ser clasificado utilizando cada pareja de modelos $\left(\lambda_{i}, \lambda_{B i}\right), i=1, \ldots, 15$. Esta prueba debe entenderse de la siguiente forma:

Clasificación de $P_{i}^{1}$ con $\left(\lambda_{i}, \lambda_{B i}\right)$. Se clasifica el primer conjunto de vectores de prueba del usuario $U_{i}$ con sus modelos asociados. Esto significa que el sistema trata la petición de un usuario válido respecto de $\lambda_{i}$, por lo que el porcentaje de acierto obtenido mide el desempeño o eficiencia del modelo generado para verificar a usuarios válidos. 
Clasificación de $P_{j}^{1}$ con $\left(\lambda_{i}, \lambda_{B i}\right), i \neq j$. Se clasifica el primer conjunto de prueba del usuario $U_{j}$, con los modelos de otro usuario $U_{i}$ distinto. Esto significa que el sistema trata con una petición de un usuario inválido respecto de $\lambda_{i}$, por lo que el porcentaje obtenido mide el error cometido por el sistema ante este tipo de solicitudes.

Esta prueba es equivalente a la realizada con Máquinas de Soporte Vectorial, cuyos resultados están reportados en las tablas 3.3 y 3.4. Esencialmente se realiza una clasificación cruzada entre los conjuntos de prueba y los modelos de cada usuario. Los resultados obtenidos para este caso, se encuentran reportados en las tablas 4.2 y 4.3 :

\begin{tabular}{|c|c|c|c|c|c|c|c|c|}
\hline$\cdot$ & $\lambda_{\mathbf{1}}, \lambda_{\mathbf{B} \mathbf{1}}$ & $\lambda_{\mathbf{2}}, \lambda_{\mathbf{B} \mathbf{2}}$ & $\lambda_{\mathbf{3}}, \lambda_{\mathbf{B 3}}$ & $\lambda_{\mathbf{4}}, \lambda_{\mathbf{B} \mathbf{4}}$ & $\lambda_{\mathbf{5}}, \lambda_{\mathbf{B 5}}$ & $\lambda_{\mathbf{6}}, \lambda_{\mathbf{B} \mathbf{6}}$ & $\lambda_{\mathbf{7}}, \lambda_{\mathbf{B} \mathbf{7}}$ & $\lambda_{\mathbf{8}}, \lambda_{\mathbf{B} \mathbf{8}}$ \\
\hline$P_{1}^{1}$ & $\mathbf{7 9 . 3 6 5}$ & 23.015 & 30.952 & 20.555 & 42.460 & 36.111 & 40.079 & 9.523 \\
\hline$P_{2}^{1}$ & 24.206 & $\mathbf{8 2 . 9 3 6}$ & 17.857 & 19.047 & 23.809 & 35.317 & 19.047 & 9.523 \\
\hline$P_{3}^{1}$ & 26.194 & 20.238 & $\mathbf{7 8 . 5 7 1}$ & 31.746 & 40.079 & 30.555 & 30.555 & 19.444 \\
\hline$P_{4}^{1}$ & 28.174 & 20.634 & 40.079 & $\mathbf{7 3 . 0 1 5}$ & 46.428 & 43.650 & 36.904 & 6.746 \\
\hline$P_{5}^{1}$ & 31.746 & 23.412 & 38.888 & 36.111 & $\mathbf{6 2 . 6 9 8}$ & 41.666 & 32.142 & 9.523 \\
\hline$P_{6}^{1}$ & 28.174 & 22.222 & 29.761 & 42.460 & 33.333 & $\mathbf{7 1 . 4 2 8}$ & 48.412 & 9.126 \\
\hline$P_{7}^{1}$ & 33.730 & 13.492 & 29.365 & 30.158 & 30.952 & 42.460 & $\mathbf{6 6 . 6 6 6}$ & 19.444 \\
\hline$P_{8}^{1}$ & 15.079 & 8.730 & 16.666 & 7.936 & 9.126 & 15.079 & 30.555 & $\mathbf{7 9 . 3 6 5}$ \\
\hline$P_{9}^{1}$ & 17.857 & 7.142 & 23.809 & 28.571 & 21.428 & 20.238 & 28.968 & 31.746 \\
\hline$P_{10}^{1}$ & 15.079 & 13.888 & 23.809 & 25.000 & 14.285 & 22.619 & 32.539 & 48.412 \\
\hline$P_{11}^{1}$ & 36.507 & 35.317 & 23.412 & 15.873 & 42.857 & 30.555 & 25.793 & 7.539 \\
\hline$P_{12}^{1}$ & 26.587 & 19.047 & 9.523 & 13.095 & 26.984 & 28.174 & 27.380 & 11.507 \\
\hline$P_{13}^{1}$ & 16.269 & 4.761 & 16.269 & 18.253 & 15.079 & 19.841 & 33.730 & 53.968 \\
\hline$P_{14}^{1}$ & 12.301 & 8.333 & 26.190 & 27.380 & 17.857 & 21.825 & 30.952 & 48.412 \\
\hline$P_{15}^{1}$ & 22.619 & 23.412 & 22.222 & 16.269 & 26.190 & 21.428 & 23.809 & 11.507 \\
\hline
\end{tabular}

Tabla 4.2: Porcentajes del sistema de verificación con GMM de los conjuntos $P_{j}^{1}$ con los modelos $\left(\lambda_{i}, \lambda_{B i}\right)$.

Como puede observarse, los mejores porcentajes de clasificación se obtienen cuando se compara el conjunto de prueba con el modelo del usuario respectivo. Esto comprueba que es posible implementar un sistema de verificación basado en GMM como clasificador. En la siguiente sección se utilizan los porcentajes obtenidos para evaluar el desempeño de este sistema. 


\begin{tabular}{|c|c|c|c|c|c|c|c|}
\hline$\cdot$ & $\lambda_{\mathbf{9}}, \lambda_{\mathbf{B 9}}$ & $\lambda_{\mathbf{1 0}}, \lambda_{\mathbf{B 1 0}}$ & $\lambda_{\mathbf{1 1}}, \lambda_{\mathbf{B 1 1}}$ & $\lambda_{\mathbf{1 2}}, \lambda_{\mathbf{B 1 2}}$ & $\lambda_{\mathbf{1 3}}, \lambda_{\mathbf{B 1 3}}$ & $\lambda_{\mathbf{1 4}}, \lambda_{\mathbf{B 1 4}}$ & $\lambda_{\mathbf{1 5}}, \lambda_{\mathbf{B 1 5}}$ \\
\hline$P_{1}^{1}$ & 16.67 & 13.095 & 42.857 & 34.920 & 11.904 & 12.698 & 25.000 \\
\hline$P_{2}^{1}$ & 8.730 & 11.507 & 45.634 & 33.333 & 12.301 & 13.095 & 36.904 \\
\hline$P_{3}^{1}$ & 20.634 & 24.206 & 21.825 & 15.873 & 14.682 & 21.031 & 21.031 \\
\hline$P_{4}^{1}$ & 26.587 & 23.412 & 23.412 & 19.047 & 11.111 & 22.619 & 11.507 \\
\hline$P_{5}^{1}$ & 21.031 & 17.063 & 36.111 & 25.793 & 12.698 & 14.285 & 27.777 \\
\hline$P_{6}^{1}$ & 18.253 & 18.650 & 25.000 & 27.380 & 15.079 & 24.603 & 16.269 \\
\hline$P_{7}^{1}$ & 20.634 & 26.587 & 19.444 & 28.571 & 30.555 & 28.571 & 14.682 \\
\hline$P_{8}^{1}$ & 25.793 & 47.222 & 6.349 & 15.873 & 54.761 & 42.857 & 6.349 \\
\hline$P_{9}^{1}$ & $\mathbf{7 9 . 3 6 5}$ & 53.968 & 10.714 & 17.857 & 48.412 & 47.619 & 11.904 \\
\hline$P_{10}^{1}$ & 53.968 & $\mathbf{7 8 . 1 7 4}$ & 10.317 & 20.238 & 51.984 & 52.777 & 7.539 \\
\hline$P_{11}^{1}$ & 10.714 & 13.095 & $\mathbf{7 3 . 8 0 9}$ & 43.650 & 10.714 & 7.539 & 32.936 \\
\hline$P_{12}^{1}$ & 14.682 & 18.650 & 48.015 & $\mathbf{7 9 . 1 9 0}$ & 20.238 & 10.317 & 40.873 \\
\hline$P_{13}^{1}$ & 44.047 & 54.365 & 12.301 & 20.238 & $\mathbf{7 0 . 2 3 8}$ & 49.603 & 10.317 \\
\hline$P_{14}^{1}$ & 48.412 & 60.714 & 9.920 & 9.920 & 55.158 & $\mathbf{7 1 . 8 2 5}$ & 5.555 \\
\hline$P_{15}^{1}$ & 9.126 & 9.920 & 38.492 & 44.841 & 15.079 & 11.507 & $\mathbf{7 8 . 1 7 4}$ \\
\hline
\end{tabular}

Tabla 4.3: (Continuación) Porcentajes del sistema de verificación con GMM de los conjuntos $P_{j}^{1}$ con los modelos $\left(\lambda_{i}, \lambda_{B i}\right)$. 


\subsubsection{Curvas DET del Sistema GMM}

Con el objetivo de realizar una comparación del desempeño de los sistemas propuestos, se utilizará el procedimiento descrito en la sección 3.2, así como los parámetros que allí se asignaron. De esta forma será posible, para ciertos valores significativos de la función DCF referida en 3.8, determinar qué sistema de clasificación presenta un mejor desempeño para la base de registros Voces-MCyTI.

Como referencia se reproduce la tabla 3.7 donde se asignan los valores de la función de costo de detección:

\begin{tabular}{|c|c|l|}
\hline Parámetro & Valor & Observaciones \\
\hline$p(H)$ & 0.015 & $\begin{array}{l}\text { Se tienen } 15 \text { usuarios válidos y un estimado total de } 1000 \\
\text { posibles. } p(H)=\frac{15}{1000} .\end{array}$ \\
\hline$p(\neg H)$ & 0.985 & $\begin{array}{l}\text { Se considera un total de 985 usuarios no válidos. } \\
p(\neg H)=1-p(H) .\end{array}$ \\
\hline$\left(w_{1}, w_{2}\right)$ & $(1,10)$ & $\begin{array}{l}\text { Se penaliza en una relación 10 a 1 el aceptar a un usuario } \\
\text { no válido. }\end{array}$ \\
\hline
\end{tabular}

Tabla 4.4: Parámetros asignados a la función DCF.

Es pertinente recordar que la curvas DET se forman utilizando los porcentajes de acierto que se encuentran en la diagonal de las tablas 4.2 y 4.3, considerados como una distribución de aciertos del sistema. Los valores de dichas tablas que no se encuentran en la diagonal son considerados como una distribución de errores del sistema, pues provienen de peticiones inválidas. Estas puntuaciones son almacenadas en conjuntos denominados TrueScores y FalseScores, respectivamente, a partir de los cuales y mediante el algoritmo del apéndice A.2, se obtienen las curvas DET. Al igual que en la evaluación de Máquinas de Soporte Vectorial, se mejoró la resolución de dichas curvas al incrementar la cardinalidad de TrueScores; para ello dado $v_{i} \in$ TrueScores, $i=1, \ldots, 15$, se generaron 500 valores con una distribución normal con media $v_{i}$ y varianza $\sigma^{2}$, por lo que la cardinalidad final de TrueScores es de 7500 .

En las figuras 4.3, 4.4 y 4.5 se esquematizan las curvas DET obtenidas para valores de $\sigma^{2}$ de $10.0,4.0$ y 2.0 respectivamente.

La tabla 4.5 resume las medidas de desempeño obtenidas mediante los puntos óptimos de error de las curvas DET (Ver ecuaciones 3.7 y 3.9 ). 


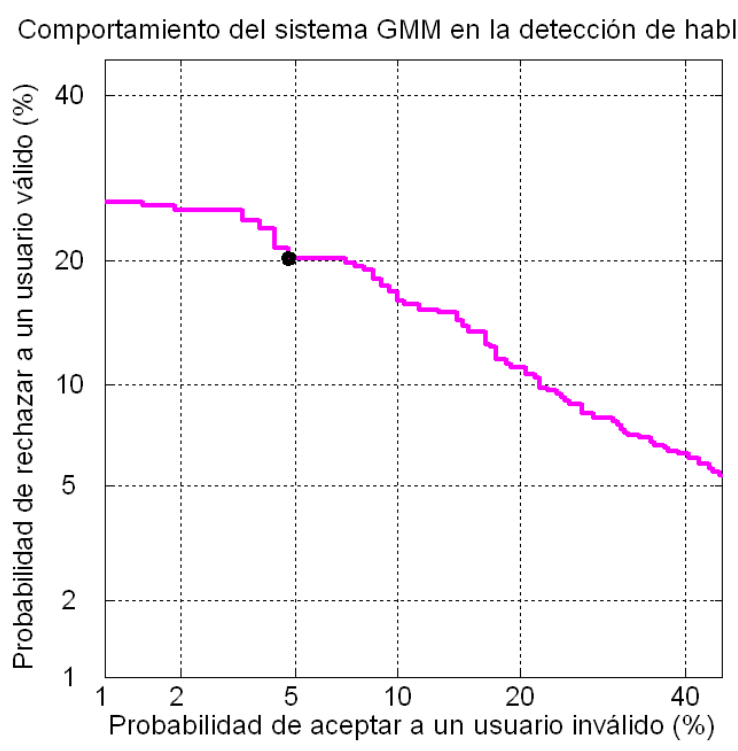

Figura 4.3: Curva DET del sistema de verificación GMM con varianza $\sigma^{2}=10.0$ en los porcentajes de acierto.

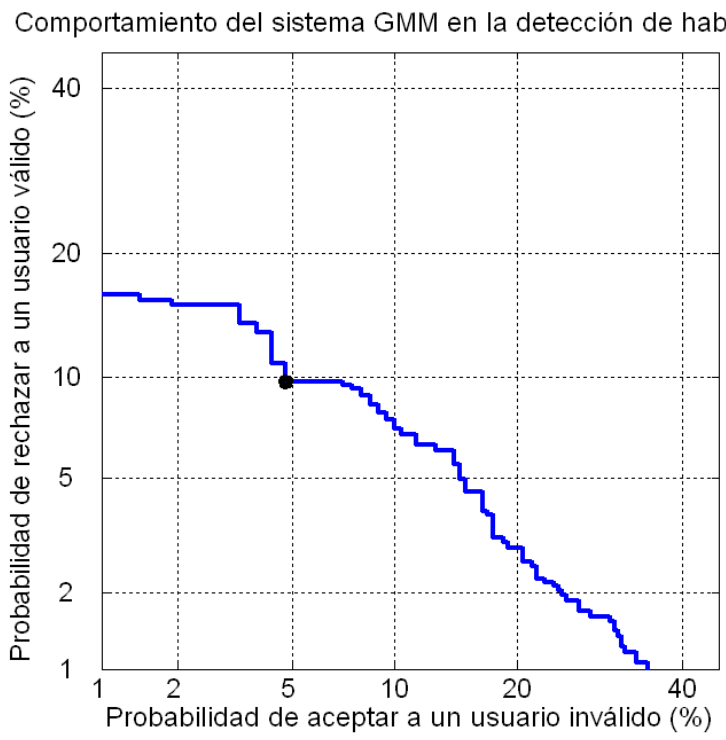

Figura 4.4: Curva DET del sistema de verificación GMM con varianza $\sigma^{2}=4.0$ en los porcentajes de acierto. 


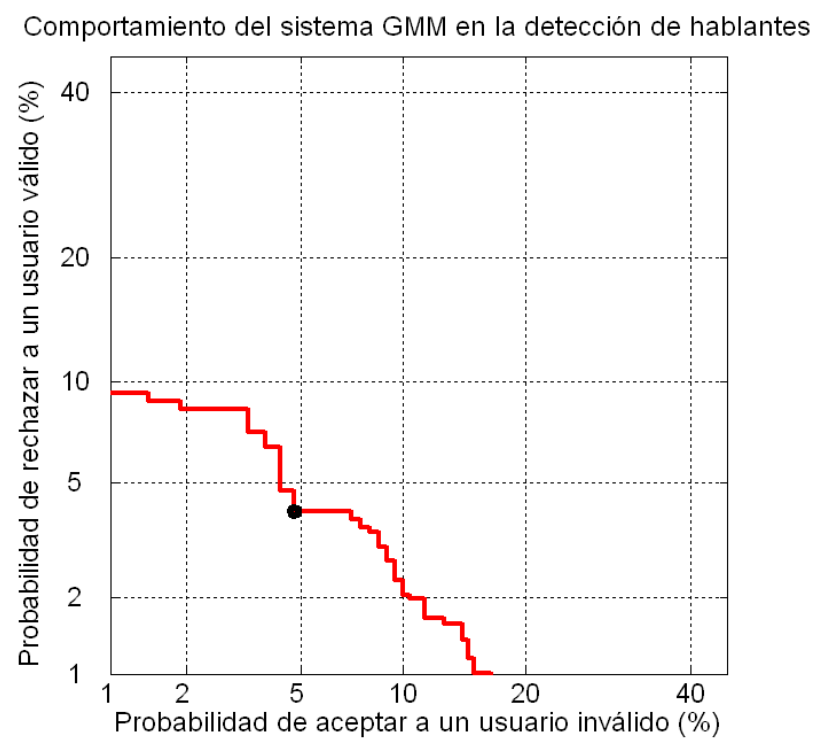

Figura 4.5: Curva DET del sistema de verificación GMM con varianza $\sigma^{2}=2.0$ en los porcentajes de acierto.

\begin{tabular}{|c|c|c|c|c|c|}
\hline Curva & Varianza & $p(R \mid H)(\%)$ & $p(A \mid \neg H)(\%)$ & $p($ Error $)$ & Desempeño ( \%) \\
\hline fig. 4.3 & 10.0 & 20.413 & 4.762 & 0.0499 & 95.01 \\
\hline fig. 4.4 & 4.0 & 9.573 & 4.762 & 0.0484 & 95.16 \\
\hline fig. 4.5 & 2.0 & 4.173 & 4.762 & 0.0475 & 95.25 \\
\hline
\end{tabular}

Tabla 4.5: Valores óptimos de error que minimizan DCF y error y desempeño asociados para el sistema GMM. 
Comparando los resultados de desempeño de la tabla 4.5 con los de la tabla 3.8, puede observarse que la clasificación mediante Máquinas de Soporte Vectorial proporciona, en cualquier caso, un mejor comportamiento que el obtenido mediante modelos de mezclas gaussianas. Sin embargo esta diferencia es, en el mejor de los casos, de tres puntos porcentuales, lo que no representa una diferencia determinante. También puede resaltarse que mientras en el sistema SVM se reducen las probabilidades de error óptimas $p(R \mid H)$ y $p(A \mid \neg H)$, al reducir la varianza $\sigma^{2}$, en el sistema GMM se mantiene constante $p(A \mid \neg H)$ y sólo se reduce $p(R \mid H)$. Esto significa que el comportamiento de ambos sistemas no es similar, lo cual se puede comprobar comparando punto a punto las curvas DET.

\subsection{Redes Neuronales Artificiales}

Una Red Neuronal Artificial (Artificial Neural Networks, ANN) es una estructura que puede ser representada matemáticamente [4]. En su forma más simple consta de sólo una unidad la cual cuenta con un conjunto de $N$ entradas $x(i) \in \mathbb{R}, i=1, \ldots, N$, y una salida $O \in \mathbb{R}$. La unidad de la red multiplica cada entrada $x(i)$ por un peso asociado $w_{i} \in \mathbb{R} \mathrm{y}$ suma todos los productos, de forma que se obtiene una combinación lineal:

$$
\sum_{i=1}^{N} w_{i} x(i) .
$$

La salida de la unidad está determinada por una función $f: D \subset \mathbb{R} \rightarrow \mathbb{R}$ de manera que

$$
O=f\left(\sum_{i=1}^{N} w_{i} x(i)\right)
$$

En la figura 4.6 se presenta un esquema de una red neuronal simple con una sola unidad. Observemos que el conjunto de entradas $x(i)$ puede ser representado como un vector $x \in \mathbb{R}^{N}$. De manera análoga el conjunto de pesos $w_{i}$ puede ser representado por un vector $w \in \mathbb{R}^{N}$.

De esta manera y con base en la notación del producto interno canónico, la ecuación 4.15 queda como

$$
O=f(w \cdot x)
$$

Supongamos la existencia de un conjunto de vectores $\left\{x_{j}\right\}_{j=1}^{l} \subset \mathbb{R}^{N}$ y un conjunto de valores $\left\{t_{j}\right\}_{j=1}^{l} \subset \mathbb{R}$, de forma que cada vector $x_{j}$ está asociado con el valor $t_{j}$ respectivo. Con esta estructura, se desea encontrar un vector de pesos $w=\left(w_{1}, \ldots, w_{N}\right) \in \mathbb{R}^{N}$ y una función $f$ de forma que

$$
f\left(w \cdot x_{j}\right)=t_{j}, \forall j=1, \ldots, l .
$$




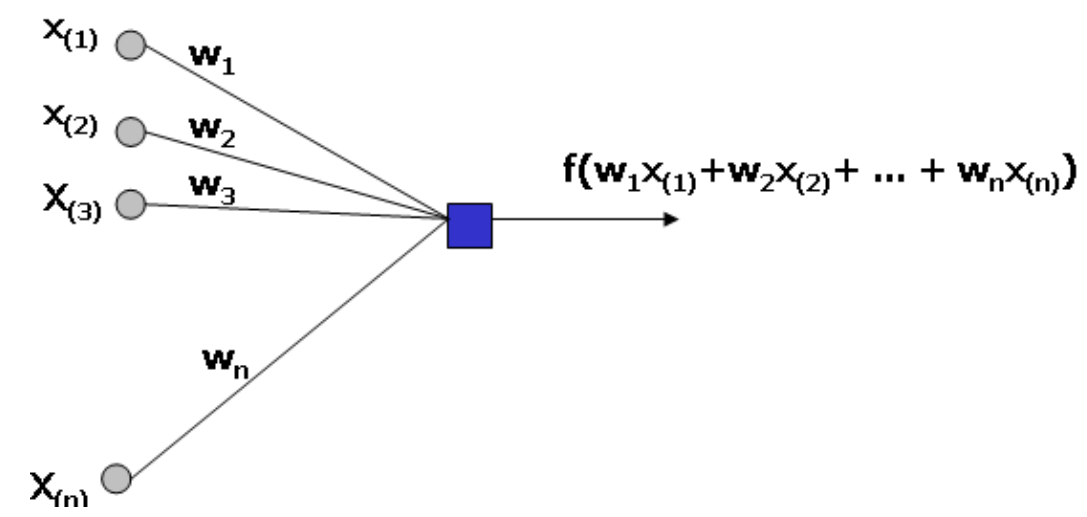

Figura 4.6: Esquema de una red neuronal con una sóla unidad de activación.

Dada una función propuesta $f$, al procedimiento para encontrar un vector de pesos $w$ que satisfaga 4.17, se le denomina entrenamiento o aprendizaje de la red. A priori, la existencia de tal vector y función no está determinada, por lo que existen diferentes propuestas de funciones $f$, así como procedimientos para observar si es posible satisfacer 4.17.

Un procedimiento consiste en minimizar el error cuadrático medio (Mean Squared Error, MSE) dado por

$$
M S E=\frac{1}{2} \sum_{j=1}^{l}\left(f\left(w \cdot x_{j}\right)-t_{j}\right)^{2} .
$$

Derivando parcialmente la ecuación 4.18 se obtiene que

$$
\begin{gathered}
\frac{\partial M S E}{\partial w_{k}}=\frac{1}{2} \sum_{j=1}^{l} 2\left(f\left(w \cdot x_{j}\right)-t_{j}\right) \frac{\partial f\left(w \cdot x_{j}\right)}{\partial w \cdot x_{j}} \frac{\partial w \cdot x_{j}}{\partial w_{k}} \\
\frac{\partial M S E}{\partial w_{k}}=\sum_{j=1}^{l}\left(f\left(w \cdot x_{j}\right)-t_{j}\right) \frac{\partial f\left(w \cdot x_{j}\right)}{\partial w \cdot x_{j}} x_{j}(k) .
\end{gathered}
$$

En particular si la función $f$ es la identidad, entonces la ecuación 4.19 toma la forma siguiente:

$$
\frac{\partial M S E}{\partial w_{k}}=\sum_{j=1}^{l}\left(w \cdot x_{j}-t_{j}\right) x_{j}(k), k=1, \ldots, N .
$$

Igualando a cero las ecuaciones 4.20 se obtiene que 


$$
\begin{gathered}
\sum_{j=1}^{l} w \cdot x_{j} x_{j}(k)=\sum_{j=1}^{l} t_{j} x_{j}(k), k=1, \ldots, N, \\
w_{1} \sum_{j=1}^{l} x_{j}(1) x_{j}(k)+\ldots+w_{N} \sum_{j=1}^{l} x_{j}(N) x_{j}(k)=\sum_{j=1}^{l} t_{j} x_{j}(k), k=1, \ldots, N .
\end{gathered}
$$

Las ecuaciones dadas en 4.21 conforman un sistema lineal de la forma $A w=B$ en las variables $w_{k}, k=1, \ldots, N$, con $A \in \mathbb{R}^{N} \times \mathbb{R}^{N}$ y $B \in \mathbb{R}^{N}$. Entonces el sistema tiene solución si la matriz $A$ tiene inversa, en cuyo caso se garantiza la existencia del vector $w$ que minimiza el error 4.18.

Es importante considerar que la ecuación 4.19 hace la suposición restrictiva de que la función $f$ es derivable lo cual, en general, no se cumple. Un método que permite la obtención de un vector $w$ sin hacer esta suposición, es el denominado Propagación Inversa (Back Propagation). Este método propone de forma aleatoria, un vector inicial $w^{0}$ cuyas entradas se acotan en un intervalo dado, posteriormente se realizan iteraciones de acuerdo a la siguiente regla de recurrencia, para cada una de las entradas $w_{k}^{0}$ del vector:

$$
w_{k}^{m+1}=w_{k}^{m}+\Delta w_{k}^{m},
$$

donde

$$
\Delta w_{k}^{m}=\eta \sum_{j=1}^{l}\left(f\left(w^{m} \cdot x_{j}\right)-t_{j}\right) x_{j}(k), k=1, \ldots, N .
$$

El parámetro $\eta$ es llamado factor de aprendizaje y puede ser ajustado en función de los resultados experimentales obtenidos. Los términos $\Delta w_{k}^{m}$ dados en 4.23 , son similares a los elementos del gradiente del error dados en 4.19, por lo que se tiene que

$$
w^{m+1} \approx w^{m}+\eta \nabla M S E .
$$

Puesto que el gradiente de una función se dirije hacia su punto de máximo crecimiento o decremento, dado un vector inicial $w^{0}$ y un valor de $\eta$ adecuados, se puede alcanzar un mínimo de $M S E$. Para establecer un criterio de paro para el algoritmo, se establece un valor mínimo de variación $d_{\min }$ entre los vectores $w^{m}$, en cada iteración:

$$
d_{\text {min }}=\left\|w^{m+1}-w^{m}\right\| .
$$

En general no es posible garantizar la convergencia del método ni que ésta sea a un óptimo global, pues esto depende de los valores iniciales. Sin embargo los resultados experimentales pueden validar dicho procedimiento. 
Cuando la función $f$ es derivable, el término $\Delta w_{k}^{m}$ en 4.22 se sustituye por

$$
\Delta w_{k}^{m}=\eta \sum_{j=1}^{l}\left(f\left(w^{m} \cdot x_{j}\right)-t_{j}\right) f^{\prime}\left(w^{m} \cdot x_{j}\right) x_{j}(k), k=1, \ldots, N,
$$

en cuyo caso se da la igualdad en 4.24. Puede observarse que el factor de aprendizaje $\eta$ realiza, en cualquier caso, un escalamiento del gradiente para ampliarlo o reducirlo. Para garantizar una aproximación adecuada a un óptimo se sugiere que $0<\eta<1$ [4]. La generalización de este desarrollo para redes neuronales multicapa es análoga.

Para utilizar redes neuronales artificiales como sistema de verificación para un usuario $U_{i}$, se consideran los conjuntos de vectores de características $E_{i}, B_{i}$ y $P_{i}$ asociados. $E_{i}$ y $B_{i}$ son utilizados para el entrenamiento de la red neuronal y $P_{i}$ para la validación de dicha estructura. En este caso sabemos que $E_{i}$ es un conjunto de vectores que corresponde a la voz del usuario, mientras que $B_{i}$ corresponde al resto o complemento de usuarios a $U_{i}$. Se desea por tanto una red neuronal $A N N_{i}$ cuya salida $O_{i}$ satisfaga lo siguiente:

$$
O_{i}(x)= \begin{cases}1 & \text { si } x \in E_{i} \\ 0 & \text { si } x \in B_{i}\end{cases}
$$

Una vez que se obtiene una red $A N N_{i}$ que satisface 4.27, para alguna función $f_{i}$ y valor $\eta_{i}$, se considera que tal red representa el modelo de la voz del usuario $U_{i}$. Para validar dicho modelo se utiliza el conjunto de prueba $P_{i}$, el cual se ingresa a la red neuronal $A N N_{i}$ y se obtienen porcentajes de acierto y error ante peticiones válidas e inválidas.

\subsubsection{Entrenamiento, Pruebas y Resultados}

Las pruebas se realizaron utilizando el simulador de redes neuronales, basado en Java, desarrollado por la Universidad de Tübingen, en Alemania, llamado Neural Network Simulator y denotado por JavaNNS (http://www-ra.informatik.uni.tuebingen.de). Este software permite, entre otras, la creación de redes neuronales multicapa y provee de herramientas para su aprovechamiento en un ambiente gráfico.

La construcción de cada red neuronal $A N N_{i}, i=1, \ldots, 15$, se basó en las características dadas en la tabla 4.6. Estos valores fueron asignados luego de realizar pruebas con diferentes estructuras, principalmente al variar el número de unidades ocultas de la red y la función de activación $f$. La figura 4.7 muestra una vista de la estructura final de cada una de estas redes.

Cada red neuronal $A N N_{i}$ fue entrenada utilizando los conjuntos $E_{i}$ y $B_{i}$ respectivos estableciendo el patrón de salida dado en 4.27. Las características más relevantes de este procedimiento se encuentran especificadas en la tabla 4.7. 


\begin{tabular}{|c|c|}
\hline Parámetro & Valor \\
\hline Unidades de entrada & 13 \\
\hline Unidades de salida & 1 \\
\hline Unidades ocultas & 4 \\
\hline Valores de activación & Lógicos $\{0,1\}$ \\
\hline Función de salida $(f)$ & Identidad \\
\hline Conexiones de red & Feed-Forward \\
\hline
\end{tabular}

Tabla 4.6: Características estructurales de las redes nueuronales $A N N_{i}$.

\begin{tabular}{|c|c|c|c|}
\hline Parámetro & Valor & Excepciones & Valor \\
\hline$\eta$ & 0.2 & $A N N_{13}$ & 0.5 \\
\hline$d_{\min }$ & 0.1 & $A N N_{13}$ & 0.35 \\
\hline Método de aprendizaje & Backpropagation & & \\
\hline Iteraciones & 10,000 & $A N N_{6}, A N N_{7}$ y $A N N_{10}$ & 20,000 \\
\hline Iniciación de $w_{k}$ & Aleatoria en $[-1,1]$ & & \\
\hline Normalización conjuntos & En el rango $[-1,1]$ & & \\
\hline Máximo error & 650 & & \\
\hline
\end{tabular}

Tabla 4.7: Valores de los principales parámetros de entrenamiento de las redes $A N N_{i}$. 


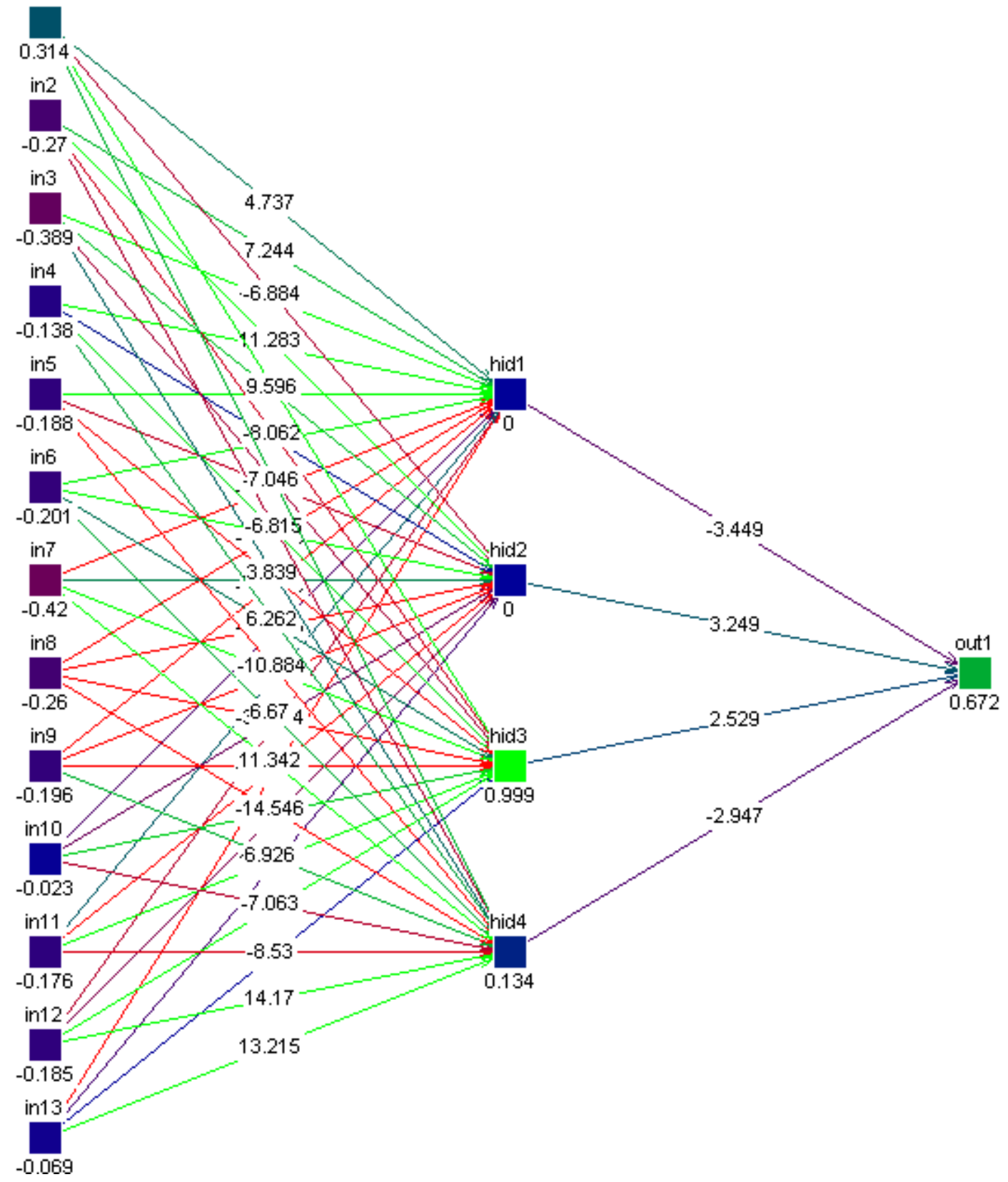

Figura 4.7: Vista de una red neuronal construida en JavaNNS para verificación de usuarios de la base Voces-MCyTI. 
El número de iteraciones se asignó considerando que el aprendizaje de la red no es significativo si el $M S E$ se mantiene constante. El valor máximo del error luego del entrenamiento se ubico en 650 unidades, para cualquier $A N N_{i}$. La figura 4.8 muestra el comportamiento típico del $M S E$ observado durante el entrenamiento, para tres valores de iniciación del vector $w_{0}$ en una misma red.

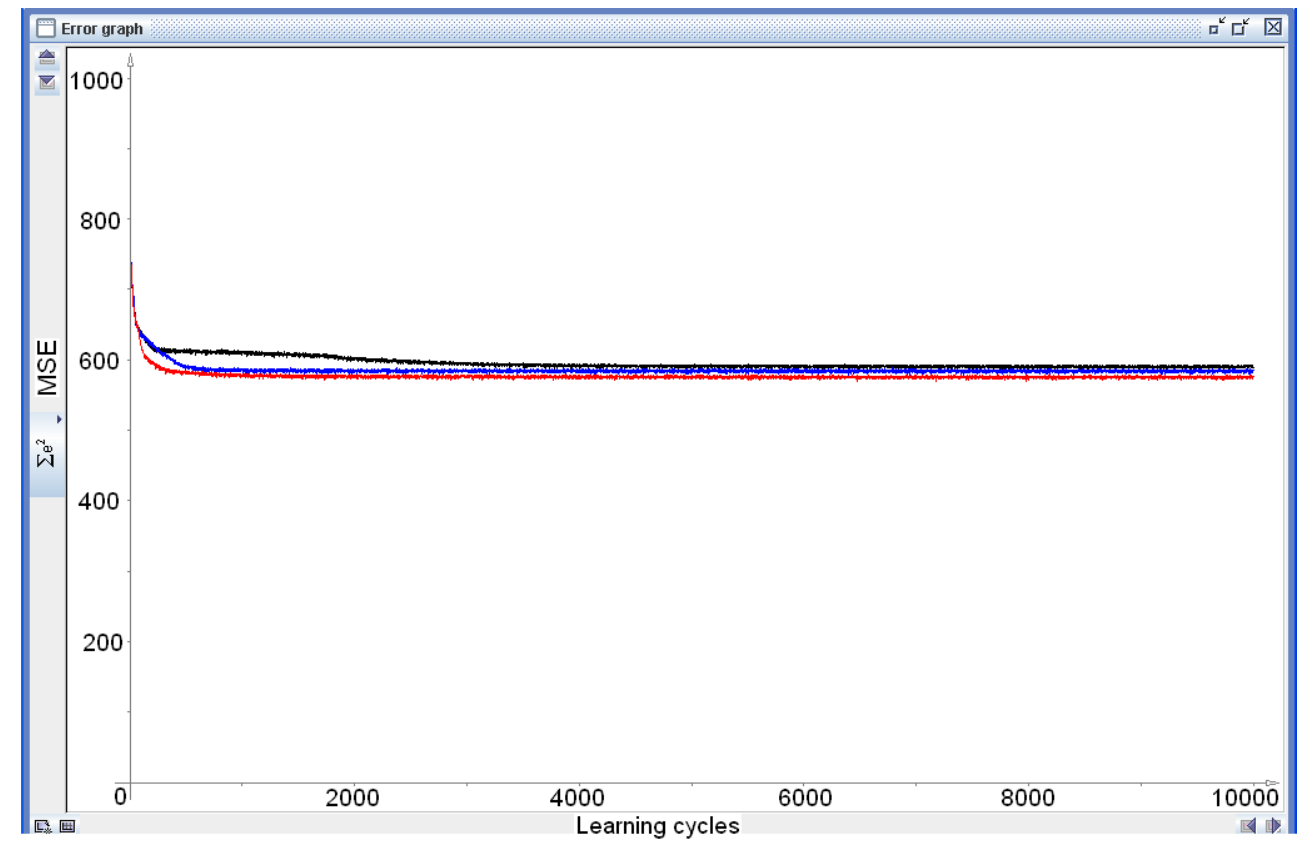

Figura 4.8: Comportamiento típico del MSE en el entrenamiento de las redes $A N N_{i}$. Cada curva representa una iniciación aleatoria del vector $w^{0}$

Una vez entrenadas las 15 redes $A N N_{i}$, se procedió a validar cada uno de estos modelos mediante los conjuntos de prueba $P_{i}^{1}$. Para ello se ingresó cada uno de estos conjuntos como entrada de cada una de las redes $A N N_{i}$. El significado de estas pruebas se da a continuación:

Clasificación de $P_{i}^{1}$ con $A N N_{i}$. Se clasifica el primer conjunto de vectores de prueba del usuario $U_{i}$ con la red $A N N_{i}$ asociada. Esto significa que el sistema trata la petición de un usuario válido respecto de $A N N_{i}$, por lo que el porcentaje de acierto obtenido mide el desempeño o eficiencia del modelo generado para verificar a usuarios válidos.

Clasificación de $P_{j}^{1}$ con $A N N_{i}, i \neq j$. Se clasifica el primer conjunto de prueba del usuario $U_{j}$, con la red de otro usuario $U_{i}$ distinto. Esto significa que el sistema trata con una petición de un usuario inválido respecto de $A N N_{i}$, por lo que el porcentaje obtenido mide el error cometido por el sistema ante este tipo de solicitudes. 


\begin{tabular}{|c|c|c|c|c|c|c|c|c|}
\hline$\cdot$ & $\mathbf{A N N}_{\mathbf{1}}$ & $\mathbf{A N N}_{\mathbf{2}}$ & $\mathbf{A N N}_{\mathbf{3}}$ & $\mathbf{A N N}_{\mathbf{4}}$ & $\mathbf{A N N}_{\mathbf{5}}$ & $\mathbf{A N N}_{\mathbf{6}}$ & $\mathbf{A N N}_{\mathbf{7}}$ & $\mathbf{A N N}_{\mathbf{8}}$ \\
\hline$P_{1}^{1}$ & $\mathbf{8 2 . 9 3 6}$ & 40.079 & 14.285 & 16.269 & 36.111 & 32.936 & 32.539 & 17.857 \\
\hline$P_{2}^{1}$ & 9.126 & $\mathbf{8 4 . 9 2 0}$ & 40.873 & 40.476 & 16.666 & 39.285 & 19.047 & 4.761 \\
\hline$P_{3}^{1}$ & 19.841 & 27.777 & $\mathbf{7 5 . 7 9 3}$ & 23.809 & 30.555 & 46.428 & 26.984 & 13.888 \\
\hline$P_{4}^{1}$ & 19.841 & 28.571 & 38.492 & $\mathbf{6 8 . 6 5 0}$ & 37.698 & 46.825 & 39.285 & 6.349 \\
\hline$P_{5}^{1}$ & 25.000 & 29.365 & 40.873 & 27.380 & $\mathbf{6 1 . 9 0 4}$ & 44.047 & 31.746 & 11.111 \\
\hline$P_{6}^{1}$ & 30.158 & 40.476 & 20.238 & 13.888 & 41.666 & $\mathbf{6 6 . 2 6 9}$ & 44.047 & 17.857 \\
\hline$P_{7}^{1}$ & 12.698 & 18.253 & 29.365 & 40.476 & 26.587 & 63.888 & $\mathbf{7 1 . 4 2 8}$ & 6.349 \\
\hline$P_{8}^{1}$ & 22.619 & 31.746 & 16.666 & 2.777 & 7.936 & 9.523 & 18.253 & $\mathbf{8 4 . 1 2 6}$ \\
\hline$P_{9}^{1}$ & 15.079 & 5.555 & 48.412 & 46.428 & 16.666 & 40.079 & 26.984 & 10.714 \\
\hline$P_{10}^{1}$ & 20.634 & 38.095 & 15.873 & 6.349 & 12.698 & 19.047 & 23.809 & 43.253 \\
\hline$P_{11}^{1}$ & 16.666 & 36.507 & 54.365 & 53.174 & 26.190 & 57.539 & 18.650 & 3.571 \\
\hline$P_{12}^{1}$ & 15.079 & 34.126 & 7.539 & 11.111 & 20.634 & 28.174 & 31.349 & 7.936 \\
\hline$P_{13}^{1}$ & 5.952 & 6.349 & 38.492 & 52.380 & 15.476 & 49.206 & 40.079 & 10.317 \\
\hline$P_{14}^{1}$ & 11.111 & 16.666 & 37.698 & 30.15 & 9.126 & 31.746 & 30.555 & 13.492 \\
\hline$P_{15}^{1}$ & 23.015 & 37.698 & 24.206 & 12.698 & 23.809 & 32.142 & 22.619 & 10.714 \\
\hline
\end{tabular}

Tabla 4.8: Porcentajes del sistema de verificación con ANN.

\begin{tabular}{|c|c|c|c|c|c|c|c|}
\hline$\cdot$ & $\mathbf{A N N}_{\mathbf{9}}$ & $\mathbf{A N N}_{\mathbf{1 0}}$ & $\mathbf{A N N}_{\mathbf{1 1}}$ & $\mathbf{A N N}_{\mathbf{1 2}}$ & $\mathbf{A N N}_{\mathbf{1 3}}$ & $\mathbf{A N N}_{\mathbf{1 4}}$ & $\mathbf{A N N}_{\mathbf{1 5}}$ \\
\hline$P_{1}^{1}$ & 34.126 & 11.111 & 1.190 & 42.857 & 41.269 & 36.111 & 46.031 \\
\hline$P_{2}^{1}$ & 5.952 & 13.492 & 44.444 & 21.825 & 14.682 & 24.603 & 21.428 \\
\hline$P_{3}^{1}$ & 24.206 & 25.000 & 3.571 & 11.507 & 24.603 & 28.571 & 25.793 \\
\hline$P_{4}^{1}$ & 34.126 & 17.857 & 5.555 & 17.857 & 29.365 & 42.063 & 17.857 \\
\hline$P_{5}^{1}$ & 27.777 & 14.682 & 5.555 & 20.238 & 34.920 & 25.396 & 27.777 \\
\hline$P_{6}^{1}$ & 42.857 & 24.206 & 1.984 & 34.920 & 40.873 & 46.428 & 40.079 \\
\hline$P_{7}^{1}$ & 22.619 & 15.079 & 5.158 & 16.269 & 44.444 & 23.809 & 13.095 \\
\hline$P_{8}^{1}$ & 47.619 & 42.460 & 0.000 & 29.761 & 40.476 & 54.761 & 23.809 \\
\hline$P_{9}^{1}$ & $\mathbf{6 6 . 6 6 6}$ & 22.222 & 7.936 & 11.507 & 51.984 & 36.507 & 8.333 \\
\hline$P_{10}^{1}$ & 57.936 & $\mathbf{7 4 . 6 0 3}$ & 0.793 & 22.222 & 38.888 & 55.952 & 24.603 \\
\hline$P_{11}^{1}$ & 10.714 & 8.730 & $\mathbf{7 3 . 0 1 5}$ & 17.857 & 6.349 & 18.650 & 15.873 \\
\hline$P_{12}^{1}$ & 19.444 & 11.111 & 5.555 & $\mathbf{7 1 . 0 3 1}$ & 29.761 & 22.619 & 46.603 \\
\hline$P_{13}^{1}$ & 30.158 & 19.047 & 11.111 & 15.873 & $\mathbf{6 5 . 0 7 9}$ & 29.761 & 9.126 \\
\hline$P_{14}^{1}$ & 40.079 & 42.460 & 1.984 & 9.523 & 48.412 & $\mathbf{7 3 . 8 0 9}$ & 7.936 \\
\hline$P_{15}^{1}$ & 14.682 & 14.285 & 5.952 & 50.000 & 30.952 & 21.031 & $\mathbf{7 8 . 9 6 8}$ \\
\hline
\end{tabular}

Tabla 4.9: (Continuación) Porcentajes del sistema de verificación con ANN. 
Los resultados obtenidos de estas pruebas se encuentran reportados en las tablas 4.8 y 4.9 .

Nuevamente, el mejor porcentaje de clasificación se obtiene cuando se compara el conjunto $P_{i}^{1}$ con su modelo respectivo $A N N_{i}$ (Valores en negritas). La evaluación del desempeño del sistema ANN se da en la siguiente sección.

\subsubsection{Curvas DET del sistema ANN}

Los aspectos relevantes de la obtención de las curvas DET para un sistema de clasificación, han sido descritos en la seccion 3.2, por lo que únicamente se mencionan aquí los apectos principales.

Los parámetros asignados a la función DCF para la obtención de las curvas DET son los mismos que los establecidos en la tabla 4.4. Al igual que para la evaluación de Máquinas de Soporte Vectorial, se mejoró la resolución de dichas curvas al incrementar la cardinalidad de TrueScores; para ello dado $v_{i} \in$ TrueScores, $i=1, \ldots, 15$, se generaron 500 valores con una distribución normal con media $v_{i}$ y varianza $\sigma^{2}$, por lo que la cardinalidad final de TrueScores es de 7500. El conjunto FalseScores consistió de los 210 valores que se encuentran fuera de la diagonal en las tablas 4.8. En las figuras 4.9, 4.10 y 4.11 se esquematizan las curvas DET obtenidas para valores de $\sigma^{2}$ de $10.0,4.0$ y 2.0 , respectivamente.

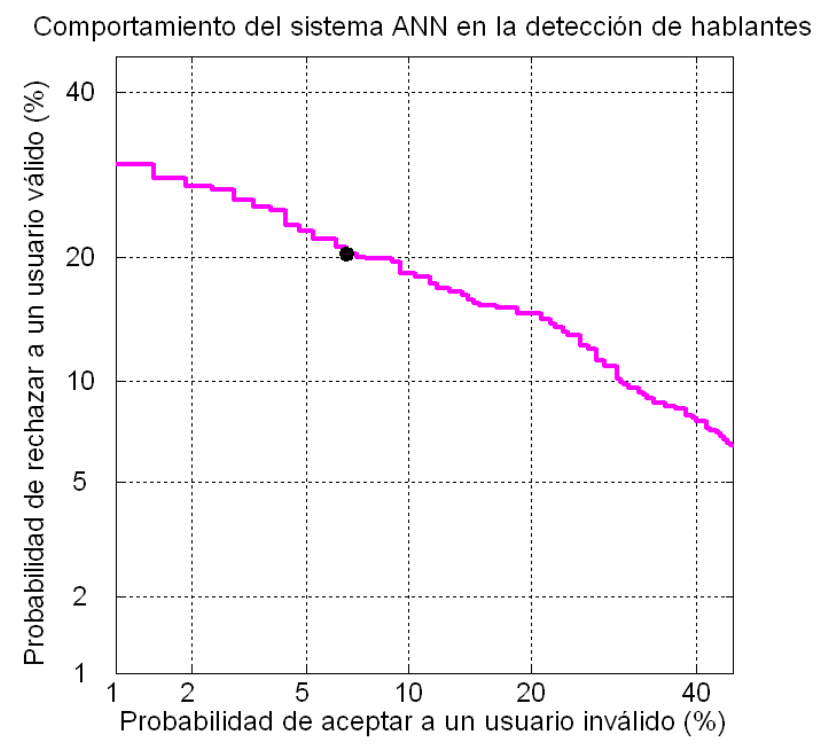

Figura 4.9: Curva DET para el sistema de verificación ANN con varianza $\sigma^{2}=10.0$ en los porcentajes de acierto. 


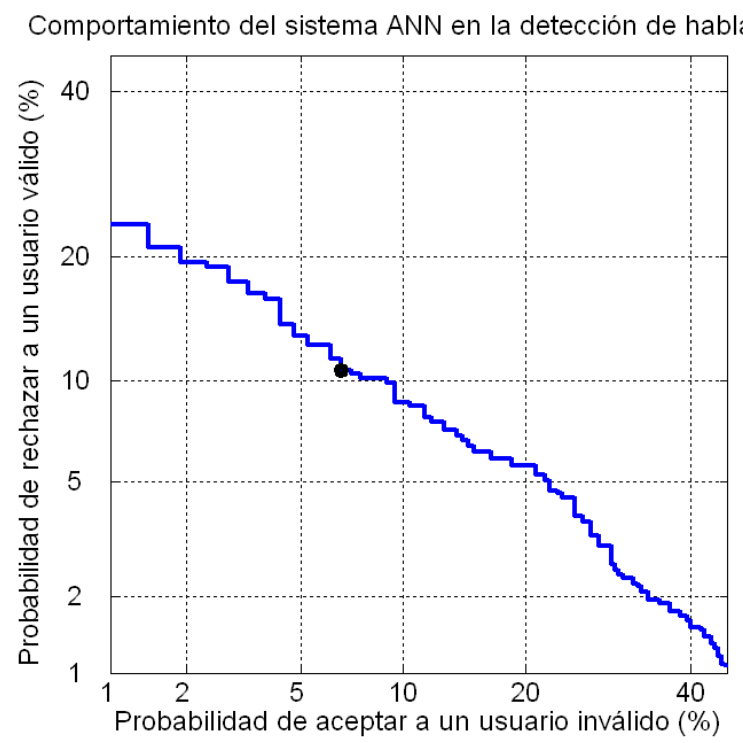

Figura 4.10: Curva DET para el sistema de verificación ANN con varianza $\sigma^{2}=4.0$ en los porcentajes de acierto.

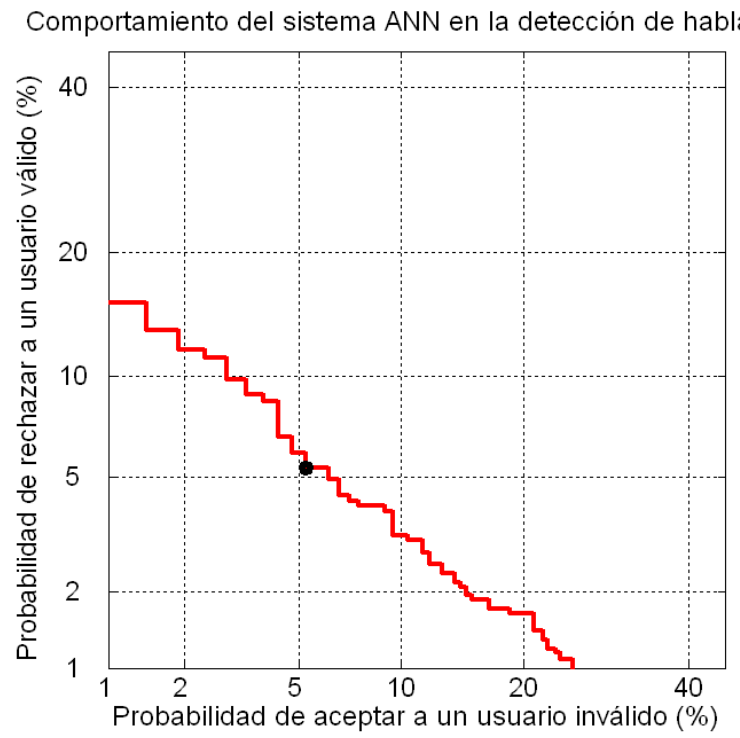

Figura 4.11: Curva DET para el sistema de verificación ANN con varianza $\sigma^{2}=2.0$ en los porcentajes de acierto. 
La tabla 4.10 resume las medidas de desempeño obtenidas mediante los puntos óptimos de error de las curvas DET (Ver ecuaciones 3.7 y 3.9).

\begin{tabular}{|c|c|c|c|c|c|}
\hline Curva & Varianza & $p(R \mid H)(\%)$ & $p(A \mid \neg H)(\%)$ & $p($ Error $)$ & Desempeño ( \%) \\
\hline fig. 4.9 & 10.0 & 20.346 & 6.666 & 0.0687 & 93.13 \\
\hline fig. 4.10 & 4.0 & 10.613 & 6.666 & 0.0672 & 93.28 \\
\hline fig. 4.11 & 2.0 & 5.346 & 5.238 & 0.0524 & 94.76 \\
\hline
\end{tabular}

Tabla 4.10: Valores óptimos de error que minimizan DCF y error y desempeño asociados para el sistema ANN.

Al comparar estos resultados de desempeño con los presentados en la tabla 3.8, se observa que nuevamente el sistema mediante Máquinas de Soporte Vectorial presenta un mejor comportamiento. Sin embargo las diferencias son, en cualquier caso, no mayores a 4.5 puntos porcentuales, lo cual representa una diferencia mínima.

A pesar de esto, el comportamiento punto a punto de las curvas DET es mejor en el sistema SVM que en ANN, lo cual significa que el sistema SVM tiene en cualquier caso, un mejor comportamiento y no sólo en los puntos óptimos. 


\section{Capítulo 5}

\section{Conclusiones y Trabajo Futuro}

\subsection{Conclusiones}

El trabajo teórico y experimental realizado en esta investigación, permite establecer las siguientes conclusiones:

- Se estableció una metodología para crear un sistema automático de verificación de hablantes, basado en Máquinas de Soporte Vectorial como sistema de clasificación.

- Se validó la metodología propuesta implementando, a partir de ésta, un sistema automático de verificación por voz. Este sistema incluyó la creación de una base de registros de voz en español llamada Voces-MCyTI, módulos de procesamiento de los registros basados en el análisis MFCC y la aplicación del sistema Libsvm, como sistema de clasificación basado en Máquinas de Soporte Vectorial. En la etapa de procesamiento se presentó y validó un supresor de silencios o pausas entre palabras. Se comprobó que dicho proceso es necesario para un correcta operación del sistema de verificación.

- Los porcentajes de clasificación obtenidos en las pruebas realizadas al sistema (Tablas 3.3 y 3.4), permiten concluir que es posible crear un sistema de verificación e incluso de identificación de personas a partir de la voz.

- Los resultados presentados en la tabla 3.5, garantizan que el sistema es estable al disminuir el número de vectores del conjunto de prueba. Los porcentajes obtenidos para peticiones válidas con conjuntos de 102 vectores, seleccionados de forma aleatoria, están por arriba del $50 \%$.

- El desempeño mostrado por el sistema y representado en las curvas DET (figuras 3.2, 3.3 y 3.4), muestra que el error disminuye al disminuir la varianza en los porcentajes de acierto. Este comportamiento indica que el desempeño del sistema mejorará, si se logra minimizar la variabilidad en los vectores de voz asociados a un mismo usuario. 
- El sistema implementado fue validado mediante su evaluación con registros de voz en tiempo real. Esta validación permitió comprobar que el sistema es independiente de las frases pronunciadas o si estas se hacen de forma secuencial. El desempeño obtenido es, en cualquier caso, muy próximo (menos de $1 \%$ ) al obtenido mediante los conjuntos de prueba $P_{i}$, formados mediante selección aleatoria. La validación incluyó muestras de voz de usuarios que no cuentan con un modelo dentro del sistema. Los porcentajes de acierto obtenidos se encuentran por encima del $50 \%$.

- Se presentó y validó un procedimiento para obtener el desempeño de sistemas automáticos de verificación. Mediante este procedimiento fue posible obtener los porcentajes para dos sistemas de clasificación adicionales; Modelos de Mezclas de Gaussianas y Redes Neuronales Artificiales. La tabla 5.1 muestra los resultados de desempeño para los tres sistemas propuestos en este trabajo.

\begin{tabular}{|c|c|c|c|}
\hline$\sigma^{\mathbf{2}}$ & SVM & GMM & ANN \\
\hline $\mathbf{1 0 . 0}$ & $97.38 \%$ & $95.01 \%$ & $93.13 \%$ \\
\hline $\mathbf{4 . 0}$ & $97.53 \%$ & $95.16 \%$ & $93.28 \%$ \\
\hline $\mathbf{2 . 0}$ & $98.07 \%$ & $95.25 \%$ & $94.76 \%$ \\
\hline
\end{tabular}

Tabla 5.1: Desempeño de tres sistemas de clasificación aplicados a la base Voces-MCyTI.

- Las gráficas dadas a continuación, presentan una comparación del error para los tres sistemas de clasificación evaluados.

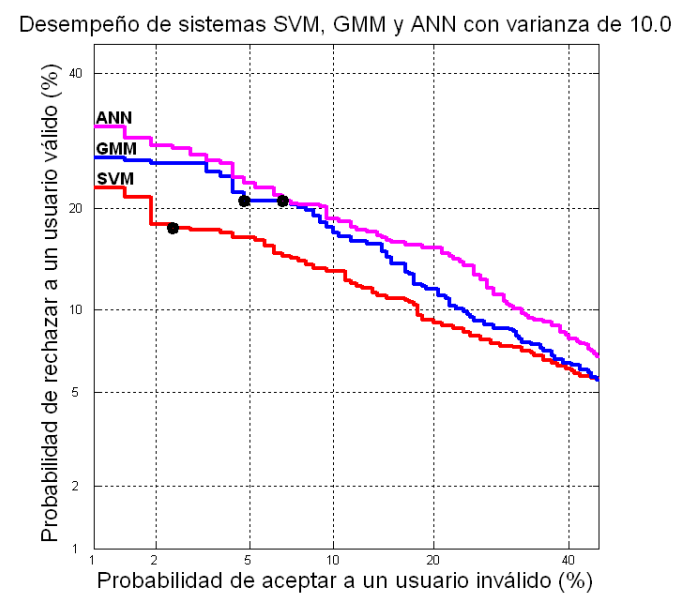

Figura 5.1: Error de tres sistemas de clasificación evaluados en la base Voces-MCyTI. $\sigma^{2}=10.0$. 


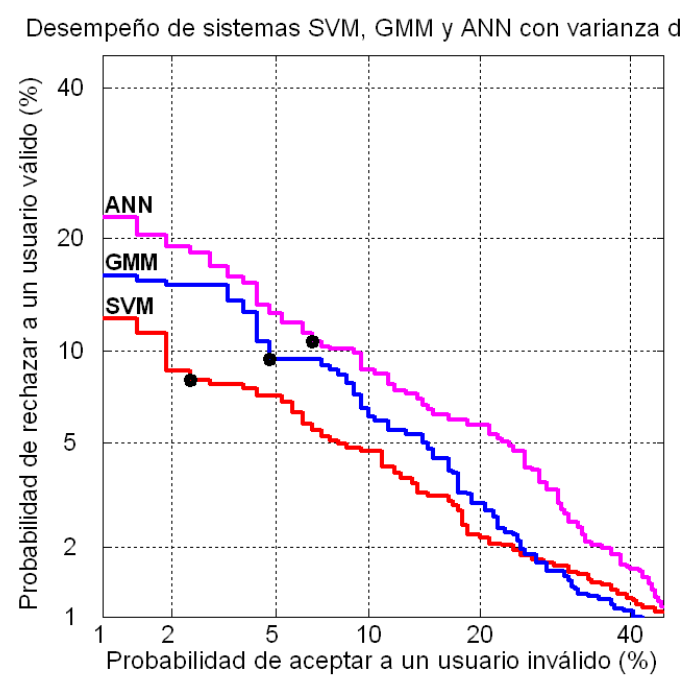

Figura 5.2: Comparación del error de tres sistemas de clasificación evaluados en la base Voces-MCyTI. $\sigma^{2}=4.0$.

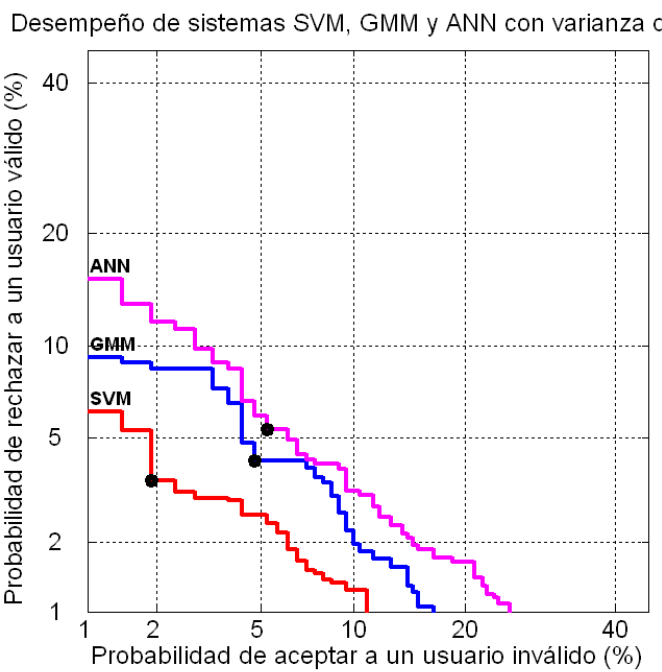

Figura 5.3: Comparación del error de tres sistemas de clasificación evaluados en la base Voces-MCyTI. $\sigma^{2}=2.0$. 
- De estos resultados se concluye que el sistema mediante Máquinas de Soporte Vectorial (SVM), tiene mejor desempeño que los sistemas GMM y ANN, aún cuando la diferencias encontradas se encuentren en un intervalo de $4.5 \%$.

- El error representado en las curvas DET de cada uno de los tres sistemas de clasificación, muestra que el sistema basado en Máquinas de Soporte Vectorial, tiene el mejor comportamiento de probabilidad de error. Esto significa que tiene un mejor manejo ante peticiones de usuarios válidos e inválidos.

Las conclusiones hechas permiten verificar el logro de los objetivos propuestos. También confirman que las Máquinas de Soporte Vectorial son una técnica exitosa que, con base en la metodología propuesta, puede mejorar el desempeño de otros sistemas de clasificación, como se mostró para los sistemas GMM y ANN. Por su relevancia en el contexto de la investigación, puede mencionarse la creación de una base de registros de voz en español, lo cual contribuye al acervo pues existen pocas referencias de este tipo de trabajos en nuestro lenguaje. En la parte de procesamiento, la propuesta y validación del supresor de silencios constituye una opción sencilla y viable que permite y mejora sustancialmente el desempeño global. Por último se resalta la contribución del trabajo en su conjunto, ya que permite una implementación directa mediante software, de un sistema automático de verificación de hablantes basado en SVM, lo cual resulta novedoso en México.

\subsection{Trabajo Futuro}

Como parte principal del trabajo a futuro propuesto, se encuentra la variación de los parámetros utilizados en el procesamiento de los registros de voz; principalmente el tiempo de cada segmento o trama en el análisis Cepstral, la atenuación y resolución del banco de filtros Mel, así como el número de coeficientes cepstrales, mismo que determina la dimensión de los vectores de características. Estas variaciones tienen el objetivo de mejorar la resolución entre las distribuciones de los porcentajes de acierto y error, del sistema mediante Máquinas de Soporte Vectorial y aumentar así su desempeño. La base Voces-MCyTI puede complementarse incrementando el número de hablantes registrados. Algunos de éstos registros pueden ser utilizados para evaluar el sistema ante peticiones de usuarios que no cuenten con un modelo dentro del mismo, en una forma más amplia. Los vectores provenientes de registros no enrolados en el sistema, pueden complementar el Background de los usuarios válidos, lo cual puede mejorar el desempeño al disminuir errores de tipo $(A, \neg H)$. Por último se propone realizar pruebas con el sistema SVM utilizando otras funciones núcleo, a fin de observar su comportamiento en la clasificación. 


\section{Apéndice A}

\section{Anexos}

\section{A.1. Notación SAMPA del Español}

\begin{tabular}{|c|c|c|}
\hline Notación & Ejemplo & Ejemplo en SAMPA \\
\hline $\mathrm{p}$ & padre & "paDre \\
\hline $\mathrm{b}$ & vino & "bino \\
\hline $\mathrm{t}$ & tomo & "tomo \\
\hline $\mathrm{d}$ & donde & "donde \\
\hline $\mathrm{k}$ & casa & "kasa \\
\hline tS & mucho & $"$ mutSo \\
\hline jj & hielo & "jjelo \\
\hline $\mathrm{f}$ & fácil & "faTil \\
\hline $\mathrm{B}(=/ \mathrm{b} /)$ & cabra & "kaBra \\
\hline $\mathrm{T}$ & cinco & "Tinko \\
\hline $\mathrm{D}(=/ \mathrm{d} /)$ & nada & "naDa \\
\hline $\mathrm{S}$ & sala & "sala \\
\hline $\mathrm{x}$ & mujer & mu"xer \\
\hline $\mathrm{G}(=/ \mathrm{g} /)$ & luego & "lweGo \\
\hline $\mathrm{m}$ & mismo & "mismo \\
\hline $\mathrm{n}$ & nunca & "nunka \\
\hline $\mathrm{J}$ & 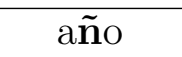 & $"$ aJo \\
\hline 1 & lejos & "lexos \\
\hline $\mathrm{L}$ & caballo & ka"baLo \\
\hline
\end{tabular}

Tabla A.1: Notación SAMPA del español. 


\begin{tabular}{|c|c|c|}
\hline Notación & Ejemplo & Ejemplo en SAMPA \\
\hline $\mathrm{r}$ & puro & "puro \\
\hline $\mathrm{rr}$ & torre & "torre \\
\hline $\mathrm{j}$ & rey/ pie & rrej/pje \\
\hline $\mathrm{w}$ & deuda / muy & "dewda/mwi \\
\hline $\mathrm{a}$ & valle & "baLe \\
\hline $\mathrm{e}$ & pero & "pero \\
\hline $\mathrm{i}$ & pico & "piko \\
\hline $\mathrm{o}$ & toro & "toro \\
\hline $\mathrm{u}$ & duro & "duro \\
\hline
\end{tabular}

Tabla A.2: (Continuación) Notación SAMPA del español.

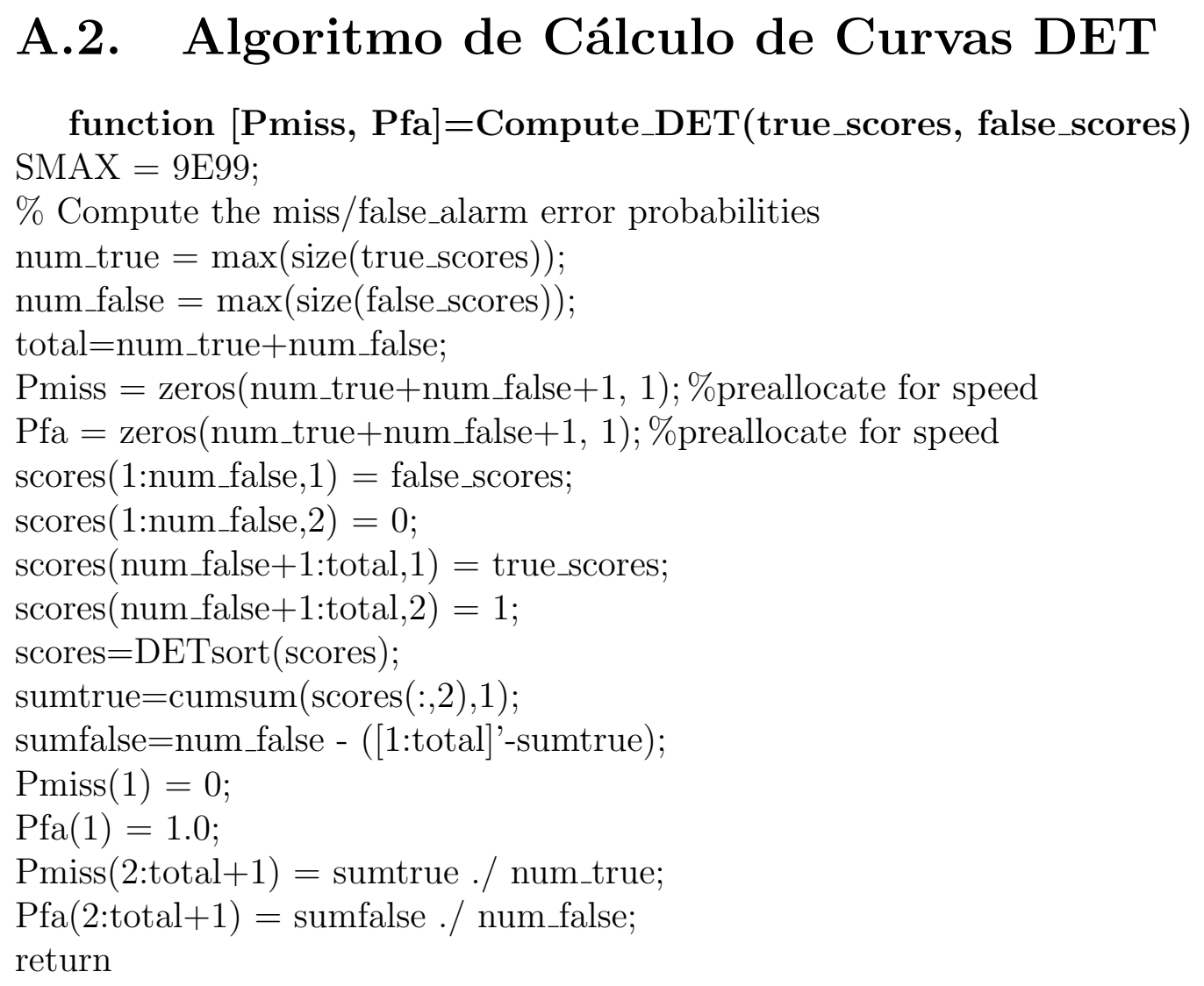

function $\mathrm{h}=$ Plot_DET (Pmiss, Pfa, plot_code, opt_thickness) Npts $=\max (\operatorname{size}($ Pmiss $))$;

if $\mathrm{Npts} \sim=\max (\operatorname{size}(\mathrm{Pfa}))$ 
error ('vector size of Pmiss and Pfa not equal in call to Plot_DET');

end

$\%$

$\%$ plot the DET

if exist('plot_code')

plot_code $=$ 'y';

end

if $\sim$ exist('opt_thickness')

opt_thickness $=0.5$;

end

Set_DET limits;

$\mathrm{h}=$ thick(opt_thickness,plot(ppndf(Pfa), ppndf(Pmiss), plot_code));

Make_DET;

\section{function Make_DET()}

pticks $=\left[\begin{array}{llllll}0.00001 & 0.00002 & 0.00005 & 0.0001 & 0.0002 & 0.0005 \ldots\end{array}\right.$

$0.0010 .0020 .0050 .010 .020 .05 \ldots$

$0.10 .20 .40 .60 .80 .9 \ldots$

$0.950 .980 .990 .9950 .9980 .999 \ldots$

$0.99950 .99980 .99990 .999950 .999980 .99999] ;$

xlabels $=\left[{ }^{\prime} 0.001^{\prime} ;{ }^{\prime} 0.002^{\prime} ;{ }^{\prime} 0.005^{\prime} ;{ }^{\prime} 0.01^{\prime} ;{ }^{\prime} 0.02{ }^{\prime} ;{ }^{\prime} 0.05 ' ; \ldots\right.$

'0.1';'0.2';'0.5';'11';'2';' 5'; ...

' $10^{\prime} ;$; 20 '; 40 ';' 60 ';' $80^{\prime}$ ';' 90 '; $;.$

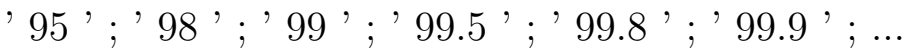

' 99.95' ; ' 99.98' ;' 99.99' ; '99.995' ; '99.998' ; '99.999'];

ylabels $=$ xlabels;

$$
\%
$$

$\%$ Get the min/max values of Pmiss and Pfa to plot

global DET_limits;

if isempty(DET_limits)

Set_DET_limits;

end

Pmiss_min = DET_limits(1);

Pmiss_max $=$ DET_limits $(2)$;

Pfa_min = DET_limits(3);

Pfa_max $=D E T \operatorname{limits}(4)$; 
$\%$

$\%$ Find the subset of tick marks to plot

ntick $=\max ($ size $($ pticks $)) ;$

for $(\mathrm{n}=$ ntick:-1:1)

if (Pmiss_min $<=\operatorname{pticks(n)})$

tmin_miss $=\mathrm{n}$;

end

if $($ Pfa_min $<=\operatorname{pticks(n)})$

tmin $f a=n$;

end

end

for $(\mathrm{n}=1$ :ntick $)$

if (pticks(n) $<=$ Pmiss_max)

tmax_miss $=\mathrm{n}$;

end

if (pticks(n) $<=$ Pfa_max) tmax_fa $=n$;

end

end

$\%$

$\%$ Plot the DET grid

set (gca, 'xlim', ppndf([Pfa_min Pfa_max $]))$;

set (gca, 'xtick', ppndf(pticks(tmin_fa:tmax_fa)));

set (gca, 'xticklabel', xlabels(tmin_fa:tmax_fa,:)); set (gca, 'xgrid', 'on');

xlabel ('Probabilidad de rechazar a un usuario válido (\%)');

set (gca, 'ylim', ppndf([Pmiss_min Pmiss_max $]))$;

set (gca, 'ytick', ppndf(pticks(tmin_miss:tmax_miss)));

set (gca, 'yticklabel', ylabels(tmin_miss:tmax_miss,:));

set (gca, 'ygrid', 'on')

ylabel ('Probabilidad de aceptar a un usuario invlido (\%)')

set (gca, 'box', 'on');

axis('square');

$\operatorname{axis(axis);}$

\%function ppndf (prob) The input to this function is a cumulative probability. The output from this function is the Normal deviate that corresponds to that probability. 


\section{Referencias}

[1] A. Higgins, L. Bahler and J. Porter, Speaker Verification Using Randomized Phrase Prompting, Digital Signal Processing, Vol. 1, No. 2, pp.89-106, 1991.

[2] A. Martin, G. Doddington, T. Kamm, M. Ordowski, M. Przybocki, The DET Curve in Assesment of Detection Task Performance, National Institute of Standards and Technology, SRI International Department of Defense, Department of Defense, USA, http://www.nist.gov/speech/.

[3] A.L. Higgins and R.E. Wholford, A New Method on Text-Independent Speaker Recognition, IEEE Proceedings of The International Conference on Acoustic, Speech and Signal Processing, Tokyo, Japan, pp. 869-872, 1986.

[4] A.K. Jain, J. Mao and K.M. Mohiuddin, Artificial Neural Networks: A Tutorial, IEEE Transactions, Vol. 29, pp. 31-44, 1996.

[5] B.H. Juang, R. Perdue and D. Thompson, Deployable Authomatic Speech Recognition Systems: Advances and Challenges, AT\&T Technical Journal, No 74(2), 1995.

[6] B.S. Atal, Effectiveness of Linear Prediction Characteristics of Speech Wave for Automatic Speaker Identification and Verification, Journal Acoustic of America, Vol. 55, No. 6, pp.1304-1312, 1974.

[7] C. Che and Q. Lin, Speaker Recognition Using Hidden Markov Models with Experiments on The YOHO Data Base, Proceedings EUROSPEECH, Madrid, Spain, pp.625-628, 1995.

[8] C.C. Tappert, N.R. Dixon, A.S. Rabinowitz and W.D. Chapma, Automatic Recognition of Continuous Speech Utilizing Dynamic Segmentation, Dual Classification, Sequential Decoding and Error Recovery, Technical Report TR-71-146, Rome Air Devices Center, Rome, NY, 1971.

[9] C.J.C. Burges, A Tutorial on Support Vector Machines for Pattern Recognition, Microsoft Research, Data Meaning and Knowledge Discovery 2, pp. 121-167, 1998. 
[10] D. Childers, R.V. Cox, R. DeMori, S. Furui, B.H. Juang, J.J. Mariani, P. Price, S. Sagayama, M.M. Sondhi, R. Weischedel, The Past, Present and Future of Speech Processing, IEEE Signal Processing Magazine, pp. 24-48, May 1998.

[11] D. Reynolds, MIT Lincoln Laboratory Site Presentation, Speaker Recognition Workshop, Maritime Institute of Technology, A. Martin, MD, 1996.

[12] D. Reynolds and B. Carlson, Text-Dependent Speaker Verification Using Decoupled and Integrated Speaker and Speech Recognizers, Proceedings EUROSPEECH, Madrid, Spain, pp. $647-650,1995$.

[13] D. Reynolds and R. Rose, Robust Text-Independent Speaker Identification Using Gaussian Mixture Speaker Models, IEEE Transactions on Speech Audio Processing, Vol. 3, No. 1, pp. 72-83, 1995.

[14] D.A. Reynolds, T.F. Quatieri, R.B. Dunn, Speaker Verification Using Adapted Gaussian Mixture Models, Digital Signal Processing, Vol. 10, pp. 19-41, 2000.

[15] D.B. Fry, Theoretical Aspects of Mechanical Speech Recognition; and P. Denes, The Design and Operation of the Mechanical Speech Recognizer, University College London, Journal of British Institution Radio Engr., No. 19(4), pp. 211-229, 1959.

[16] D.R. Reddy, An Approach to Computer Speech Recognition by Direct Analysis of The Speech Wave, Technical Report C549, Computer Science Department, Stanford University, September 1966.

[17] F. Bimbot, J.F. Bonastre, C. Fredouille, G. Gravier, I. Magrin-Chagnolleau, S. Meignier, T. Merlin, J. Ortega-García, D. Petrovzka-Delacrétaz, D.A. Reynolds, A Tutorial on Text-Independent Speaker Verification, EURASIP Journal on Applied Signal Processing 4, pp. 430-451, 2004.

[18] F. Itakura, Minimum Prediction Residual Applied to Speech Recognition, IEEE Transactions on Acoustics, Speech and Signal Processing, ASSP-23(1), pp. 67-72, February 1975.

[19] F. Jelinek, L.R. Bahl and R.L. Mercer, Design of a Linguistic Statistical Decoder for the Recognition of Continuous Speech, IEEE Transactions on Information Theory, IT-21, pp.250-256, 1975.

[20] F.K. Soong, A.E. Rosemberg, L.R. Rabiner and B.H. Juang, A Vector Quantization Approach to Speaker Recognition, Proceedings of The International Conference on Acoustic, Speech and Signal Processing, Tampa, FL, pp. 387-390, 1985. 
[21] G.R. Doddington, Speaker Recognition, Identifying People by Their Voices, IEEE Proceedings, Vol. 73, pp. 1651-1664, November 1985.

[22] H. Chi-Wei, Ch. Chih-Chung, L. Chih-Jen, A Practical Guide to Support Vector Classification, http://www.csie.ntu.edu.tw/ cjlin/papers/guide.

[23] H. Fengei and W. Bingxi, Text-Independent Speaker Verification Using Speaker Clustering and Support Vector Machines, IEEE Proceedings of The International Conference on Signal Processing, 2002.

[24] H. Sakoe, Two Level DP Matching - A Dynamic Programming Based Pattern Matching Algorithm for Connected Word Recognition, IEEE Transactions on Acoustics, Speech and Signal Processing, ASSP-27, pp. 588-595, December 1979.

[25] H. Sakoe and S. Chiba, Dynamic Programming Algorithm Optimization for Spoken Word Recognition, IEEE Transactions on Acoustics, Speech and Signal Processing, ASSP26(1), pp. 43-49, February 1978.

[26] H.F. Olson and H. Belar, Phonetic Typewriter, Journal of Acoustic Society of America, No. 28(6), pp. 1072-1081, 1956.

[27] J. Attili, M. Savic and J. Campbell, A TM32020-Based Real Time, Text-Independent, Automatic, Speaker Verification System, IEEE Proceedings of The International Conference on Acoustic, Speech and Signal Processing, New York, pp. 599-602, 1988.

[28] J. Colombi, D. Ruck, S. Rogers, M. Oxley and T. Anderson, Cohort Selection and Word Grammar Effects for Speaker Recognition, IEEE Proceedings of The International Conference on Acoustic, Speech and Signal Processing, Atlanta, GA, pp. 85-88, 1996.

[29] J. Naik, Speaker Verification: A Tutorial, IEEE Communication Magazine, Vol. 28, pp.42-48, January 1990.

[30] J. Ortega-García, J. González-Rodríguez, V. Marrero-Aguiar, A Large Speech Corpus in Spanish for Speaker Characterization and Identification, Speech Communication, 1999.

[31] J. Suzuki and K. Nakata, Recognition of Japanese Vowels - Preliminary to Recognition of Speech, Journal of Radio Research Lab, No. 37(8), pp. 193-212, 1961.

[32] J.D. Markel, S.B. Davis, Text-Independent Speaker Recognition from a Large Linguistically Uncosntrained Time-Spaced Database, IEEE Transactions on Acoustic Speech, Signal Processing, Vol. ASSP-27, No. 1, pp. 74-82, 1979.

[33] J.G. Proakis, Digital Communications, Ed. McGraw Hill, Fourth ed., pp. 239. 
[34] J.P. Campbell Jr., Speaker Recognition: A Tutorial, IEEE Proceedings, Vol. 85, No. 9, September 1997.

[35] J.W. Forgie and C.D. Forgie, Results Obtained from a Vowel Recognition Computer Program, Journal of Acoustic Society of America, No. 31(11), pp. 211-229, 1959.

[36] K. Daoudi, J. Louradour, A Novel Strategy for Speaker Verification Based on SVM Classification of Pairs of Sequences, IRIT-CNRS, Toulouse, France, 2007.

[37] K.H. Davis, R. Biddulph and S. Balashek, Automatic Recognition of Spoken Digits, Journal of Acoustic Society of America, No. 24, pp. 637-642, 1952.

[38] K.P. Li and E.H. Wrench, Jr., Text-Independent Speaker Recognition with Short Utterances, IEEE Proceedings of The International Conference on Acoustic, Speech and Signal Processing, Boston, MA, pp. 555-558, 1983.

[39] L.R. Rabiner, S.E. Levinson, A.E. Rosemberg and J.G. Wilpon, Speaker Independent Recognition of Isolated Words Using Clustering Techniques, IEEE Transactions on Acoustics, Speech and Signal Processing, ASSP-27, pp. 336-349, August 1979.

[40] M. Seltzer, SPHINX III Signal Processing Front End Specification, CMU Speech Group, 1999.

[41] N.Z. Tishby, On The Application of Mixture Hidden Markov Models to Text Independent Speaker Recognition, IEEE Transactions on Acoustic, Speech and Signal Processing, Vol. 39, No. 3, pp. 563-570, 1991.

[42] Q. Miao, H-Z. Huang and X. Fan, A Novel Hybrid System with Neural Networks and Hidden Markov Models in Fault Diagnosis, Lecture Notes on Artificial Intelligence 4293, pp. 513-521, MICAI-2006.

[43] R. Schwartz, S. Roucos and M. Beroutti, The Application of Probability Density Estimation on Text Independent Speaker Identification, Proceedings of The International Conference on Acoustic, Speech and Signal Processing, Paris, France, pp. 1649-1652, 1982.

[44] S. Furui, Cepstral Analysis Technique for Automatic Speaker Verification, IEEE Transactions on Acoustic, Speech and Signal Processing, Vol. ASSP-29, pp. 254-272, 1981.

[45] S. Raghavan, G. Lazarou and J. Picone, Speaker Verification Using Support Vector Machines, Center for Advanced Vehicular Systems, Missisipi State University, 2006. 
[46] T. Kinnunen, Spectral Features for Automatic Text-Independent Speaker Recognition, Licenciate's Thesis, University of Joensuu, Department of Computer Science, Finland, 2003.

[47] T. Sakai and S. Doshita. The Phonetic Typewriter, Information Processing, IFIP Congress, Munich, 1962.

[48] T.B. Martin, A.L. Nelson and H.J. Zadell, Speech Recognition by Feature Abstraction Techniques, Technical Report AL-TDR-64-176, Air Force Avionics Lab, 1964.

[49] T.K. Vintsyuk, Speech Discrimination by Dynamic Programming, Kibernetika, No. 4(2), pp.81-88, January-February 1968.

[50] V. Wan, Speaker Verification Using Support Vector Machines, Ph.D Thesis, University of Sheffield, Department of Computer Science, United Kingdom, 2003.

[51] V. Wan and S. Renals, Speaker Verification Using Sequence Discriminant Support Vector Machines, IEEE Transactions on Speech and Audio Processing, Vol 13, No. 2, 2005.

[52] V.M. Velichko and N.G. Zagoruyco, Automatic Recognition of 200 Words, Journal of Man-Machine Studies, No. 2, pp.223, June 1970.

[53] W.M. Campbell, A SVM/HMM System for Speaker Recognition, IEEE Proceedings of The International Conference on Acoustic, Speech and Signal Processing, 2003.

[54] W.M. Campbell, D.E. Sturim and D.A. Reynolds, Support Vector Machines Using GMM Supervectors for Speaker Verification, IEEE Signal Processing Letters, 2006.

[55] W.M. Campbell, J.P. Campbell, D.A. Reynolds, D.A. Jones and T.R. Leek, High Level Speaker Verification with Support Vector Machines, IEEE Proceedings of The International Conference on Acoustic, Speech and Signal Processing, 2004.

[56] X. Dong and W. Zhahoui, Speaker Recognition Using Continuous Density Support Vector Machines, IEEE Electronic Letters, Vol. 37, No. 17, pp. 1099-1101, 2001. 